Alma Mater Studiorum - Università di Bologna IN COTUTELA CON LUDWIG-MAXIMILIANS-UNIVERSITÄT MÜNCHEN

Dottorato di Ricerca in Fisica

Ciclo XXXI

Settore concorsuale di afferenza: 02/A2

Settore scientifico disciplinare: FIS/02

\title{
On the corpuscular theory of gravity
}

\author{
Presentata da: Andrea Giusti
}

Coordinatore Dottorato:

Supervisore:

Prof.ssa Silvia Arcelli

Prof. Roberto Casadio

Supervisore:

Prof. Georgi Dvali

Esame finale anno 2018 



\section{Summary}

The aim of this thesis is to provide a general description of the corpuscular theory of gravity. After reviewing some of the major conceptual issues emerging from the semiclassical and field theoretic approaches to Einstein's gravity, we present a synthetic overview of two novel (and extremely intertwined) perspectives on quantum mechanical effects in gravity: the horizon quantum mechanics formalism and the classicalization scheme. After this preliminary discussion, we then proceed with implementing the latter to several different scenarios, namely self-gravitating systems, the early Universe, and galactic dynamics.

Concerning the first scenario, we start by describing the generation of the Newtonian potential as the result of a coherent state of toy (scalar) gravitons. After that we employ this result to study some features of the gravitational collapse and to argue that black holes can be thought of a self-sustained quantum states, at the critical point, made of a large number soft virtual gravitons. We then refine this simplified analysis by constructing an effective theory for the gravitational potential of a static spherical symmetric system up to the first post-Newtonian correction. Additionally, we employ the horizon quantum mechanics formalism to study the causal structure emerging from the corpuscular scenario. Finally, we present a short discussion of corpuscular black holes in lower dimensional spaces.

After laying down the basics of corpuscular black holes, we present a generalization of the aforementioned arguments to cosmology. Specifically, we first introduce a corpuscular interpretation of the de Sitter spacetime. Then we use it as the starting point for a corpuscular formulation of the inflationary scenario and to provide an alternative viewpoint on the dark components of the $\Lambda \mathrm{CDM}$ model.

The key message of this work is that the corpuscular theory of gravity offers a way to unify most of the experimental observations (from astrophysical to galactic and cosmological scales) in a single framework, solely based on gravity and baryonic matter. 


\section{Zusammenfassung}

Das Ziel dieser These ist eine allgemeine Beschreibung der "Korpuskeltheorie" zu liefern. Nach der Überprüfung einiger der wichtigsten konzeptionellen Probleme die sich aus den semiklassischen Ansätzen und den Ansätzen der Quantenfeldtheorie zu Einsteins Schwerkraft ergeben, präsentieren wir einen synthetischen Überblick über zwei neuartige (und extrem verflochtene) Perspektiven über quantenmechanische Effekte in der Gravitation: der Formalismus der Quantenmechanik des Horizonts und das Klassifizierungsschema. Nach dieser Vorbesprechung fahren wir dann mit der Umsetzung von verschiedenen Szenarien fort, nämlich Selbstgravitationssysteme, das frühe Universum und die galaktische Dynamik.

Was das erste Szenario betrifft, so beschreiben wir zunächst die Generierung des Newtonschen Potenzials als Ergebnis eines kohärenten Zustands von vereinfachten (skalaren) Gravitons. Danach verwenden wir dieses Ergebnis um einige Merkmale des Gravitationskollapses zu untersuchen. Weiterhin nutzen wir das Ergebnis zu argumentieren, dass schwarze Löcher an einen selbst nachhaltigen Quantenzustand gedacht werden können, an der kritischen Stelle, die aus einer großen Anzahl weicher virtueller Gravitons besteht. Diese vereinfachte Analyse verfeinern wir dann, indem wir eine wirksame Theorie für das Gravitationspotenzial eines statistischen kugelförmigen symmetrischen Systems bis zur ersten Post-Newtonschen Korrektur konstruieren. Darüber hinaus verwenden wir den Formalismus der Quantenmechanik des Horizonts, um die kausale Struktur zu untersuchen, die aus dem korpuskulären Szenario entsteht. Schließlich stellen wir eine kurze Diskussion über korpuskuläre schwarze Löcher in unteren dimensionalen Räumen vor.

Nachdem wir die Grundlagen der korpuskulären schwarzen Löcher gelegt haben, stellen wir eine Verallgemeinerung der oben genannten Argumente zur Kosmologie vor. Konkret führen wir zunächst eine korpuskuläre Interpretation der de Sitter Raumzeit ein. Dann nutzen wir das als Ausgangspunkt für eine korpuskuläre Formulierung des inflationären Szenarios und weiterhin, um einen alternativen Standpunkt zu den dunklen Komponenten des $\Lambda$ CDM Modells zu bieten.

Die Kernbotschaft dieser Arbeit ist, dass die "Korpuskeltheorie" eine Möglichkeit bietet, die meisten experimentellen Beobachtungen (von astrophysikalischen bis galaktischen und kosmologischen Skalen) in einen einzigen Rahmen zu vereinen, der sich ausschließlich auf der Schwerkraft und der Baryonischen Materie basiert. 
To the loving memory of the greatest man. I have ever fonown, my grandfacter. Marcellino, and to my grandmother Margherita 



\section{Contents}

1 Introduction 1

1.1 Motivations and outline . . . . . . . . . . . . . . . 1

1.2 Notations and Conventions $\ldots \ldots \ldots \ldots \ldots \ldots$

1.3 This Thesis . . . . . . . . . . . . . . . . . . 8

1.4 Report on academic activities $\ldots \ldots \ldots \ldots$

1.5 Complete List of Publications $\ldots \ldots \ldots \ldots$

\begin{tabular}{|lll}
2 & Semiclassical gravity and related issues & 13
\end{tabular}

$2.1 \quad$ Black hole termodynamics and the need for a quantum theory . . . . . . 13

2.2 Quantum fields on black hole spacetimes . . . . . . . . . . . . . . 16

2.3 Remarks on gravity as a quantum field theory . . . . . . . . . . . . . 22

\begin{tabular}{|lll}
\hline 3 & Alternative perspectives on quantum effects in gravity & 27
\end{tabular}

3.1 Horizon quantum mechanics $\ldots \ldots \ldots \ldots \ldots \ldots$

3.1 .1 Trapped surfaces and event horizons in general relativity . . . . . 28

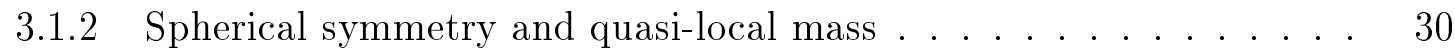

3.1 .3 HQM for spherical systems $\ldots \ldots \ldots \ldots \ldots \ldots \ldots \ldots \ldots$

3.1 .4 HQM for rotating systems $\ldots \ldots \ldots \ldots \ldots \ldots$

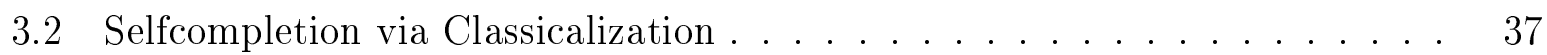

3.2 .1 Black Hole's Quantum $N$-Portrait . . . . . . . . . . . . . 40

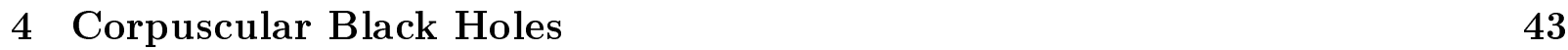

4.1 Black holes as self-sustained quantum states . . . . . . . . . . . . . 43

4.2 Gravitational collapse . . . . . . . . . . . . . . . . . . . . 47

4.3 Corpuscular post-Newtonian theory . . . . . . . . . . . . . . 50

$4.3 .1 \quad$ Classical effective scalar theory $\ldots \ldots \ldots \ldots \ldots$

4.3 .2 Quantum uplifting of the model . . . . . . . . . . . . . . 54

4.4 Rotating and non-singular black holes . . . . . . . . . . . . . . 57

4.4 .1 Quantum harmonic black holes . . . . . . . . . . . . . . 57

4.4 .2 Hayward spacetime $\ldots \ldots \ldots \ldots \ldots \ldots$

$4.4 .3 \quad$ Kerr spacetime $\ldots \ldots \ldots \ldots \ldots \ldots$

4.5 Lower dimensional corpuscular gravity $\ldots \ldots \ldots \ldots$ 
\begin{tabular}{|lll}
5 & Corpuscular cosmology & 67
\end{tabular}

5.1 Corpuscular de Sitter space . . . . . . . . . . . . . . . . . 67

5.2 Slow-roll inflation and domestic inflaton . . . . . . . . . . . . 71

5.3 Cold dark matter and corpuscular dark force $\ldots \ldots \ldots \ldots . \ldots 78$

6 Conclusions and outlook $\quad 85$

\begin{tabular}{|lr}
\hline A A note on the Fierz-Pauli kinetic term & 89
\end{tabular}

\begin{tabular}{lr}
\hline Acknowledgments & 91
\end{tabular} 


\section{List of Figures}

$2.1 \quad$ Tree-level scattering of two $\Phi$ fields of mass $m$ mediated by a graviton. . . 25

$2.2 \quad \Phi$-loop in the graviton propagator. . . . . . . . . . . . 25

$3.1 \quad$ Plot of $P_{\mathrm{BH}}$ as a function of the mass of the particle. . . . . . . . . . 35

$4.1 \quad$ Harmonic potential with $\mu=\omega=1$ and $d=5$. . . . . . . . . . . 58

4.2 Hayward's metric function $f(r)$ for different values of the ADM mass $M$, keeping $\ell$ fixed. . . . . . . . . . . . . . . 59

$5.1 \quad$ Starobinsky inflationary potential. . . . . . . . . . . . . . 72 


\section{Chapter 1}

\section{Introduction}

I do not know what I may appear to the world; but to myself, I seem to have been only like a boy playing on the seashore, and diverting myself now and then in finding a smoother pebble or prettier shell than ordinary, while the great ocean of truth lay all undiscovered before me.

Sir Isaac Newton

\subsection{Motivations and outline}

General relativity (GR) |1] can fairly be regarded as the most successful theory in the history of physics, having outlasted both its original advocates and most of its critics, enduring the test of time and our capabilities to observe deviations from its predictions at both astrophysical and very large scales. However, indications of its incompleteness are hidden in plain sight.

According to GR, we can understand the nature of gravity in purely geometrical terms. Specifically, from special relativity [2] we learn that space and time should not be regarded as separate entities, but rather they constitute a unique geometrical object, commonly named Minkowski spacetime [3], which consists of a flat four-dimensional pseudoRiemannian manifold with signature $(-,+,+,+)$. Then, the fundamental idea behind GR is the intent to extend the special theory of relativity, which is indeed focussed on inertial reference frames, to all systems of reference. To be more precise, the key concept is that all systems of reference should be equivalent with respect to the formulation of the fundamental laws of physics. The main consequence of this assumption is that gravity is no longer an action-at-a-distance force, as in Newton's theory [4], but rather an alteration of the curvature of the spacetime in response to the local presence of an energy distribution 
of some sort. Thus, the gravitational field is now measured by the spacetime curvature and its fluctuations propagate at the speed of light.

Black holes are one of the most important predictions of GR whose existence, together with gravitational waves, has recently been confirmed by the LIGO-VIRGO collaboration [5, 6]. These very peculiar objects are regions of the spacetime exhibiting an extremely strong gravitational field such that nothing can escape from their interior. The boundary of such a region is called the event horizon of the black hole. This name comes from the fact that, since not even light can escape from the interior or the boundary of a black hole, the events (point of the spacetime manifold) inside the horizon are causally disconnected form the rest of the Universe. In other words, there is no future-directed causal (timelike or null) path that can connect an event inside the black hole to an event outside the horizon. However, as anticipated, the breakdown of the classical description is indeed hiding in plain sight. Let us take, for example, the Schwarzschild spacetime, which represents the first black hole solution discovered in GR $|7-10|$, one can easily see that the location of the horizon represents a coordinate singularity |9,10|, which can easily be removed by an appropriate choice of coordinates, whereas the "center" of the black hole displays a singular behaviour that is reflected also in the Kretschmann scalar. Since a scalar function is diffeomorphism invariant, this divergence cannot be removed by a coordinate transformation, and therefore it is referred to as curvature singularity (or "true" singularity). The emergence of curvature singularities is the benchmark for the breakdown of the classical GR description of gravity.

Note that this singular behaviour seems to appear only when we are close enough to the core of the Schwarzschild black hole, so in a very small region of the spacetime where all the energy content associated to the black hole is supposed to converge. It is therefore reasonable to think that the emergence of this singularity might be connected with the fact that we are completely ignoring the contribution of quantum mechanical effects, and that gravity itself might change a lot its behaviour at those scales (or energy densities). This very simple argument suggests the need for a quantum theory of gravity, that should reconcile Einstein's gravity and quantum mechanics at very large energies or, equivalently, at very small length scales. The first attempt to put together quantum mechanics and black hole physics dates back to the seventies, with the seminal work by Hawking [11] who contributed to kick start a completely new line of research, that we now know as quantum field theory on curved spacetimes. The main idea behind this approach is that, in certain regimes, we can consider Einstein's gravity as a classical background on which quantum fields can live. The main prediction of this picture is that black holes are not inert objects, but rather they slowly evaporate by emitting thermal radiation, known as Hawking radiation. However, this evaporation comes at a price. Indeed, consider the gravitational collapse of a matter source to form a black hole which then evaporates away completely through the Hawking process, leaving behind only some thermal radiation. Quantum mechanically, the collapsing matter can be arranged into a pure quantum state. Nonetheless, the final state emerging from a complete evaporation of the system would be a mixed state, since the radiation is purely thermal. Such a process would then violate the unitarity of the time evolution of a quantum system, which is one of the fundamental postulates of quantum 
mechanics. This conceptual issue is known as the information paradox. Besides, needless to say that this perspective on semiclassical gravity does not really tackle the problem of curvature singularities, which are still left to be taken care of by quantum gravitational effects.

Cosmology is also another natural playground for Eintein's gravity. Indeed, a clear example is given by the observed expansion of the Universe that, at large scales, can be correctly modeled by introducing the cosmological constant term in the vacuum Einstein field equations. This was first suggested by Einstein in [12,13], though it was ultimately disregarded until the late 1930s, once Hubble's discovery |14 became widely accepted. However, the physical nature of this cosmological constant contribution is still rather obscure and it is therefore referred to as dark energy $[15$ in the literature. Yet, in spite of the success of this new term in accounting for the observed expansion of the Universe, GR together with baryonic matter and dark energy is not capable of providing an accurate physical explanation of many astronomical observations, such as the rotation curves of spiral galaxies $16-20 \mid$. The simplest way to proceed is then to assume the existence of a non-baryonic matter component which can be perceived only through its gravitational effects [20]. This is indeed the starting point that led to the modern formulation of the standard model of Big Bang cosmology, also known as $\Lambda$ CDM model (i.e. $\Lambda$ Cold Dark Matter model). According to the latter, our Universe can be split into three components: dark energy, accounting for almost the $68 \%$ of the total energy density; cold dark matter, responsible for the $27 \%$; baryonic matter, representing around the $5 \%$ of the energy of the Universe.

This very useful model leaves, however, some conceptual questions unanswered. Indeed, it offers no justification for the fact that, statistically, the Universe appears to be homogeneous and isotropic at very large scales, this is known as the horizon problem. Besides, the $\Lambda C D M$ model cannot give a suitable explanation for the observed flatness of the Universe without a great deal of fine tuning; this issue takes the name of flatness problem. All the issues mentioned above find their natural solution in the theory of cosmic inflation [21- 24], that assumes the existence of a phase of exponential expansion of the spacetime in the early Universe.

The aim of this work is to present an alternative perspective on semiclassical gravity, mostly inspired by the effective field theory approach to strongly coupled theories. Indeed, by means of some key ideas coming from the classicalization scenario, we are going to discuss how we can unify black hole physics, dark matter phenomenology, and the inflationary scenario in a single framework, the corpuscular model of gravity.

This thesis is therefore organized as follows:

In Chapter 2 we review some of the key features and issues of semiclassical gravity, with particular regard for black hole physics. Specifically, we start by recalling how black hole thermodynamics highlights the need for the reconciliation of gravity with the quantum theory (Section 2.1). Then we provide a brief discussion of the main features of quantum field theory on black holes spacetimes (Section 2.2), with particular regard for the Hawking effect and related issues. Finally, we conclude the Chapter by recalling a few ideas about the bottom-up construction of the gravitational interaction in quantum field theory, based 
on the weak field expansion of the Einstein-Hilbert action, and we also present a few remarks on the issue of the non-renormalizablility of the theory (Section 2.3).

In Chapter 3, after a brief overview on the Wilsonian approach to non-renormalizable theories, we introduce two alternative perspectives on semiclassical gravity, namely the horizon quantum mechanics formalism and the UV completion of gravity by classicalization. In detail, in Section 3.1 we review the general formalism of the horizon quantum mechanics for both spherically symmetric and axisymmetric systems together with an example of application of this technique to a single Gaussian source. In Section 3.2 we summarize the fundamental notions laying at the foundations of the classicalization scheme. Taking profit of the latter we then introduce the quantum $N$-portrait for black holes (Section 3.2.1), which represents the first root of the corpuscular theory of gravity.

In Chapter 4 we then describe the corpuscular picture for black holes and the gravitational collapse. Precisely, in Section 4.1 we present the fundamental groundwork that allows to formally understand black holes as self-sustained quantum states. We then use the key ideas coming from this argument to study some features of the gravitational collapse in this corpuscular picture. This is done by means of a reasoning based on energy balances (Section 4.2). In Section 4.3 we then refine the discussion presented in Section 4.2 by constructing an effective theory for the gravitational potential of a static spherical symmetric system up to the first post-Newtonian correction. In Section 4.4, after stressing the strict relation between the corpuscular model and the horizon quantum mechanics, we discuss the likelihood for the Hayward and Kerr spacetimes to emerge, as effective geometries, in the corpuscular scenario. Finally, in Section 4.5 we discuss the behaviour of the corpuscular model of black holes for lower dimensional spacetimes, specifically for the $(1+1)$-dimensional case.

In Chapter 5 we show how cosmic inflation and the observed phenomenology usually attributed to cold dark matter naturally emerge from a corpuscular description of the Universe at cosmological scales. In particular, in Section 5.1 we provide a corpuscular description of the de Sitter space, in relation with both the cosmological constant term and quadratic gravity scenario. In Section 5.2 we then employ this result to argue that the dynamics predicted by the Starobinsky model of inflation emerges naturally, in pure gravity, from the corpuscular scenario. After that, we use again the argument described in Section 5.1 and assume the existence of a cosmological condensate, that we argue to be responsible for the dark energy content of our Universe. Then we take profit of the latter to understand the phenomenology usually attributed to cold dark matter, in the $\Lambda \mathrm{CDM}$ model, as the response of the whole condensate to the local presence of baryonic matter (Section 5.3).

Finally, in Chapter 6, we conclude with remarks and hints for future research. 


\subsection{Notations and Conventions}

Indices. In this work Greek indices takes values $\mu, \nu, \ldots=0,1,2,3$ while purely spatial labels are denoted by Latin letters $i, j, k, \ldots=1,2,3$. Repeated upper and lower Greek letters are summed over, i.e.

$$
A^{\mu} B_{\mu} \equiv \sum_{\mu=0}^{3} A^{\mu} B_{\mu}
$$

while, when dealing with equations involving only spatial objects, we will tend to keep all indices as upper indices.

The notation $\boldsymbol{A}$ indicates a spatial (3-dimensional) vector, with components $\boldsymbol{A}=$ $\left(A^{1}, A^{2}, A^{3}\right)=\left\{A^{i}\right\}$. For 4 -vectors we will instead use ordinary Latin letters, unless indicated otherwise, e.g. $X$ whose component are then given by $X=\left(X^{0}, X^{1}, X^{2}, X^{3}\right)=$ $\left(X^{0}, X^{i}\right)=\left\{A^{\mu}\right\}$.

Metric. We will adopt the "mostly plus convention" for all Lorentzian manifolds. Thus, the Minkowski metric $\eta_{\mu \nu}$, in Cartesian coordinates, reads

$$
\eta_{\mu \nu}=\operatorname{diag}(-1,+1,+1,+1)=\left(\begin{array}{cccc}
-1 & 0 & 0 & 0 \\
0 & +1 & 0 & 0 \\
0 & 0 & +1 & 0 \\
0 & 0 & 0 & +1
\end{array}\right)
$$

besides, for any Lorentzian manifolds $\mathcal{M}$ equipped with a metric $g_{\mu \nu}$, i.e. $(\mathcal{M}, g)$, we have that

$$
g_{\mu \nu}=(-,+,+,+), \quad g_{\mu \nu} g^{\mu \nu}=4,
$$

where $g^{\mu \nu}$ indicates the inverse of $g_{\mu \nu}$, and that

$$
A_{\mu}=g_{\mu \nu} A^{\nu}, \quad \text { and } \quad A \cdot B:=g_{\mu \nu} A^{\mu} B^{\nu}=A^{\mu} B_{\mu}=A_{\mu} B^{\mu}
$$

where - here denotes the scalar product induced by the metric $g_{\mu \nu}$.

It is also worth recalling that, in our notation, the 0-component of a 4-dimensional index (i.e. Greek letters) refers to the time-component of the corresponding object.

For 3-dimensional vectors on the Euclidean space $\left(\mathbb{R}^{3}, \delta\right)$ we have that

$$
\boldsymbol{A} \cdot \boldsymbol{B}=A^{i} B^{i}=\delta_{i j} A^{i} B^{j}=\delta_{i j} A_{i} B_{j}=\delta^{i j} A^{i} B^{j},
$$

with $\delta_{i j}$ denoting the Kronecker delta, i.e.

$$
\delta_{i j}=\operatorname{diag}(+1,+1,+1)=\left(\begin{array}{ccc}
+1 & 0 & 0 \\
0 & +1 & 0 \\
0 & 0 & +1
\end{array}\right)
$$

again in Cartesian coordinates. 
It is also useful to stress that, on Minkowski we have that $X=\left(X^{0}, X^{i}\right) \equiv X^{\mu}$ and

$$
X_{\mu}=\eta_{\mu \nu} X^{\nu}=\left(-X^{0}, X^{i}\right) .
$$

Differential operators. On Minkowski it is possible to define the 4-derivative

$$
\partial_{\mu}:=\left(\frac{1}{c} \frac{\partial}{\partial t}, \frac{\partial}{\partial x^{i}}\right) \equiv\left(\partial_{0}, \partial_{i}\right),
$$

from which one can infer that $\partial^{\mu}=\eta^{\mu \nu} \partial_{\nu}=\left(-\partial_{0}, \partial_{i}\right)$. One can then define the (flat) d'Alembert operator as

$$
:=\partial_{\mu} \partial^{\mu}=\eta^{\mu \nu} \partial_{\mu} \partial_{\nu}=-\frac{1}{c^{2}} \frac{\partial^{2}}{\partial t^{2}}+\Delta
$$

where $\Delta:=\operatorname{div} \boldsymbol{\nabla}=\partial_{i} \partial_{i}$ is the Laplacian.

For a generic Lorentzian manifold $(\mathcal{M}, g)$ we can define the Levi-Civita connection $\nabla$, for which we recall that

$$
\nabla_{\alpha} A^{\mu}=\partial_{\alpha} A^{\mu}+\Gamma_{\alpha \beta}^{\mu} A^{\beta}, \quad \nabla_{\alpha} A_{\mu}=\partial_{\alpha} A_{\mu}-\Gamma_{\alpha \mu}^{\beta} A_{\beta}, \quad \nabla_{\alpha} g_{\mu \nu}=0,
$$

where $\nabla_{\alpha}$ denotes the covariant derivative with respect to the coordinate basis $\partial_{\alpha}$ and $\Gamma_{\mu \nu}^{\lambda}$ are the Christoffel symbols, given by

$$
\Gamma_{\mu \nu}^{\lambda}=\frac{1}{2} g^{\lambda \rho}\left(\partial_{\mu} g_{\nu \rho}+\partial_{\nu} g_{\mu \rho}-\partial_{\rho} g_{\mu \nu}\right)
$$

Furthermore, we can define the d'Alembert operator on a curved spacetime as $\square:=g^{\mu \nu} \nabla_{\mu} \nabla_{\nu}$

Main geometrical objects. We write the Riemann tensor as

$$
R_{\mu \rho \nu}^{\lambda}=\partial_{\rho} \Gamma_{\mu \nu}^{\lambda}-\partial_{\nu} \Gamma_{\mu \rho}^{\lambda}+\Gamma_{\mu \nu}^{\eta} \Gamma_{\rho \eta}^{\lambda}-\Gamma_{\mu \rho}^{\eta} \Gamma_{\nu \eta}^{\lambda},
$$

from which we define the Ricci tensor $R_{\mu \nu}=R_{\mu \lambda \nu}^{\lambda}$, whose trace gives the curvature (Ricci) scalar $R=g^{\mu \nu} R_{\mu \nu}$. It is also worth recalling that the Riemann tensor can be decomposed as

$$
R_{\mu \rho \nu}^{\lambda}=\frac{1}{2}\left(\delta_{\rho}^{\lambda} R_{\mu \nu}-g_{\mu \rho} R_{\nu}^{\lambda}-\delta_{\nu}^{\lambda} R_{\mu \rho}+g_{\mu \nu} R_{\rho}^{\lambda}\right)-\frac{R}{6}\left(\delta_{\rho}^{\lambda} g_{\mu \nu}-\delta_{\nu}^{\lambda} g_{\mu \rho}\right)+C_{\mu \rho \nu}^{\lambda}
$$

where $C^{\lambda}{ }_{\mu \rho \nu}$ denotes the Weyl (or conformal curvature) tensor.

Einstein-Hilbert action and $f(R)$-theories. When dealing with the quantum field theoretic approach to gravity we will make use of the Einstein-Hilbert action in the form

$$
S_{\mathrm{EH}}=-\frac{c^{4}}{16 \pi G_{\mathrm{N}}} \int \mathrm{d}^{4} x \sqrt{-\operatorname{det} g} R,
$$


whereas, when dealing with modified theories of gravity, we will define the Einstein-Hilbert action as

$$
S_{\mathrm{EH}}=+\frac{c^{4}}{16 \pi G_{\mathrm{N}}} \int \mathrm{d}^{4} x \sqrt{-\operatorname{det} g} R .
$$

Notice that in the latter case we have changed the global sign of the action with respect to the first definition. This does not really change much if no matter contribution is involved. Indeed, these two representations are, in general, equivalent provided that we change the sign in the functional definition of the stress-energy tensor.

A generic $f(R)$-theory is therefore defined in terms of the action functional

$$
S=\frac{c^{4}}{16 \pi G_{\mathrm{N}}} \int \mathrm{d}^{4} x \sqrt{-\operatorname{det} g} f(R)+S_{\mathrm{M}}\left[g_{\mu \nu}, \Psi\right]
$$

where $S_{\mathrm{M}}\left[g_{\mu \nu}, \Psi\right]$ is the matter action and $\Psi$ collectively denotes the matter fields. The corresponding equations of motion are then given by

$$
f^{\prime}(R) R_{\mu \nu}-\frac{1}{2} f(R) g_{\mu \nu}-\left[\nabla_{\mu} \nabla_{\nu}-g_{\mu \nu} \square\right] f^{\prime}(R)=\frac{8 \pi G_{\mathrm{N}}}{c^{4}} T_{\mu \nu},
$$

where

$$
T_{\mu \nu}:=\frac{-2}{\sqrt{-\operatorname{det} g}} \frac{\delta S_{\mathrm{M}}}{\delta g^{\mu \nu}},
$$

denotes the energy-momentum tensor while the prime indicates the differentiation with respect to the argument.

Units. In this work we will mostly set the speed of light $c=1$, but we will keep both $\hbar$ and $G_{\mathrm{N}}$ explicit. Now, recalling the definitions of Planck mass and Planck length, i.e.

$$
m_{\mathrm{P}}=\sqrt{\frac{\hbar c}{G_{\mathrm{N}}}}, \quad \ell_{\mathrm{P}}=\sqrt{\frac{\hbar G_{\mathrm{N}}}{c^{3}}},
$$

then setting $c=1$ we find

$$
G_{\mathrm{N}}=\frac{\ell_{\mathrm{P}}}{m_{\mathrm{P}}}, \quad \hbar=m_{\mathrm{P}} \ell_{\mathrm{P}}
$$

\section{Summary of conventions and notations.}

c Speed of light, usually set to one

$G_{\mathrm{N}} \quad$ Newton's constant; $G_{\mathrm{N}}=\ell_{\mathrm{P}} / m_{\mathrm{P}}$

$\hbar \quad$ Planck constant; $\hbar=m_{\mathrm{P}} \ell_{\mathrm{P}}$

$\mathscr{H}^{+} \quad$ Future event horizon

$\mathscr{I}^{ \pm} \quad$ Future/past null infinity

$J^{ \pm}(U)$ Causal future/past of the region $U$

$T_{\mu \nu} \quad$ Energy-momentum tensor

$\rho \quad$ Energy density

$p \quad$ Pressure, perfect fluid

$N_{\mathrm{G}} \quad$ Number of gravitons

$\lambda_{\mathrm{m}} \quad$ Compton-de Broglie wavelength

$\epsilon$

\begin{tabular}{|ll}
$T_{\mathrm{H}}$ & Hawking temperature \\
$S_{\mathrm{BH}}$ & Bekenstein-Hawking entropy \\
$S_{\mathrm{EH}}$ & Einstein-Hilbert $(\mathrm{EH})$ action \\
$(2 \chi)^{-1}$ & Pre-factor EH action; $\chi=8 \pi G_{\mathrm{N}} / c^{4}$ \\
$U_{\mathrm{N}}$ & Newtonian potential energy \\
$U_{\mathrm{PN}}$ & post-Newtonian potential energy \\
$H$ & Hamiltonian \\
$\mathcal{H}=0$ & Hamiltonian constraint of cosmology \\
$\Lambda$ & Cosmological constant \\
$L_{\Lambda}$ & de Sitter horizon \\
$A_{(\alpha \beta)}$ & $A_{(\alpha \beta)}=\left(A_{\alpha \beta}+A_{\beta \alpha}\right) / 2$ \\
$A_{[\alpha \beta]}$ & $A_{[\alpha \beta]}=\left(A_{\alpha \beta}-A_{\beta \alpha}\right) / 2$
\end{tabular}




\subsection{This Thesis}

This thesis is based on the author's work conducted from November 2015 and August 2018 at the University of Bologna, under the supervision of Prof. Roberto Casadio, and at the Ludwig-Maximilians-Universität München, under the supervision of Prof. Georgi Dvali. Specifically, most of this manuscript is based on the following references:

1. R. Casadio, A. Giugno and A. Giusti. Matter and gravitons in the gravitational collapse. Physics Letters B 763 (2016), 337-340.

2. R. Casadio, A. Giugno and A. Giusti. Global and local horizon quantum mechanics. Gen. Rel. Grav. 49 (2017), 32.

3. R. Casadio, A. Giugno, A. Giusti and O. Micu. Horizon quantum mechanics of rotating black holes. Eur. Phys. J. C 77 (2017), 322.

4. R. Casadio, A. Giugno, A. Giusti and M. Lenzi. Quantum corpuscular corrections to the Newtonian potential. Phys. Rev. D 96 (2017), 044010.

5. M. Cadoni, R. Casadio, A. Giusti, W. Mück and M. Tuveri. Effective Fluid Description of the Dark Universe. Physics Letters B 776 (2018), 242-248.

6. R. Casadio, A. Giugno and A. Giusti. Corpuscular slow-roll inflation. Phys. Rev. D 97 (2018), 024041.

7. A. Giugno, A. Giusti and A. Helou. Horizon quantum fuzziness for non-singular black holes. Eur. Phys. J. C 78 (2018), 208.

8. M. Cadoni, R. Casadio, A. Giusti and M. Tuveri. Emergence of a dark force in corpuscular gravity. Phys. Rev. D 97 (2018), 044047.

9. R. Casadio, A. Giugno, A. Giusti and O. Micu. Horizon quantum mechanics: spherically symmetric and rotating sources. Foundations of Physics 48 (2018), 1204-1218.

10. R. Casadio, A. Giusti and R. Rahim. Horizon quantum mechanics for spheroidal sources. Europhysics Letters 121 (2018), 60004.

11. R. Casadio, A. Giusti and J. Mureika. Lower dimensional corpuscular gravity and the end of black hole evaporation. arXiv:1805.10444.

12. A. Giugno and A. Giusti. Domestic corpuscular inflaton. arXiv:1806.11168.

13. R. Casadio, A. Giugno, A. Giusti and M. Lenzi. Quantum Formation of Primordial Black holes. arXiv:1810.05185.

14. R. Rahim, A. Giusti and R. Casadio. The marginally trapped surfaces in spheroidal space-times. Int. J. Mod. Phys. D 28 (2019), 1950021. 


\subsection{Report on academic activities}

Since November 1, 2015 I have been a PhD student in Physics (XXXI cycle) at the University of Bologna, under the supervision of Prof. Roberto Casadio. Starting from January 26, 2017 (date of the last signature of the Cotutelle Agreement) I have become a joint PhD student between the University of Bologna and the Ludwig-Maximilians-Universität München. Since then, I have been carrying out my research activities under the supervision of Prof. Roberto Casadio (University of Bologna) and Prof. Georgi Dvali (LudwigMaximilians-Universität München).

During the last three years, I have focussed on two different research lines: semiclassical gravity, under the supervision of Prof. Casadio and Prof. Dvali, and mathematical aspects of theoretical and applied physics. The latter, in particular, consists of a series of side projects mostly unrelated to the main scopes of my $\mathrm{PhD}$ studies. As a result of this endeavour I have been able to publish 30 scientific article (26 of which have already been accepted for publication in international journals), 14 of which directly related to the content of this dissertation. It is also worth stressing that, upon evaluation of the quality and extent of my scientific production, I was awarded the 2017 Augusto Righi prize by the Italian Physical Society (Società Italiana di Fisica - SIF). Concerning my side work in mathematical physics, I have contributed to the formulation and consequent development of the research project "Analysis of complex biological systems" (Principal Investigator: Dr. Luigi Funzo, University of Naples, Federico II), financed by the Italian National Group for Mathematical Physics (GNFM) and the Italian National Institute of High Mathematics (INdAM) within the scheme of the "Young Researchers Project" award.

Within the scopes of my PhD studies, I have also attended and completed five $\mathrm{PhD}$ courses (Advanced Statistics, Complements of Quantum Mechanics, Complements of Condensed Matter Physics, Complements of Nuclear and Subnuclear Physics, Quantum Physics and Gravitation), a summer school on Mathematical Physics (Ravello Summer School, September 2016), several mandatory seminar activities promoted by the PhD programme in Physics, an online course on "safety measures in the workplace", an online course aimed at improving "soft skills" and several seminars (both at the University of Bologna and LMU Munich) on theoretical physics. I have also had the opportunity to attend (around) 15 international conferences and workshops, 5 of which as Invited Speaker. 


\subsection{Complete List of Publications}

1. R. Garra, A. Giusti, F. Mainardi and G. Pagnini. Fractional relaxation with timevarying coefficient. Fract. Calc. Appl. Anal. 17 (2014), 424-439.

2. T. De Lorenzo, A. Giusti and S. Speziale. Non-singular rotating black hole with a time delay in the center. Gen. Rel. Grav. 48 (2016), 31.

3. A. Giusti and F. Mainardi. A dynamic viscoelastic analogy for fluid-filled elastic tubes. Meccanica 51 (2016) 2321.

4. A. Giusti and F. Mainardi. On infinite series concerning zeros of Bessel functions of the first kind. Eur. Phys. J. Plus 131 (2016), 206.

5. R. Casadio, A. Giugno and A. Giusti. Matter and gravitons in the gravitational collapse. Physics Letters B 763 (2016), 337-340.

6. I. Colombaro, A. Giusti and F. Mainardi. A class of linear viscoelastic models based on Bessel functions. Meccanica 52 (2017), 825.

7. R. Casadio, A. Giugno and A. Giusti. Global and local horizon quantum mechanics. Gen. Rel. Grav. 49 (2017), 32.

8. I. Colombaro, A. Giusti and F. Mainardi. On the propagation of transient waves in a viscoelastic Bessel medium. Z. Angew. Math. Phys. 68 (2017), 62.

9. A. Giusti. On infinite order differential operators in fractional viscoelasticity. Fract. Calc. Appl. Anal. 20 (2017), 854-867.

10. R. Casadio, A. Giugno, A. Giusti and O. Micu. Horizon quantum mechanics of rotating black holes. Eur. Phys. J. C 77 (2017), 322.

11. I. Colombaro, A. Giusti and F. Mainardi. On transient waves in linear viscoelasticity. Wave Motion 74 (2017), 191-212.

12. R. Casadio, A. Giugno, A. Giusti and M. Lenzi. Quantum corpuscular corrections to the Newtonian potential. Phys. Rev. D 96 (2017), 044010.

13. A. Giusti and I. Colombaro. Prabhakar-like fractional viscoelasticity. Comm. Nonlin. Sci. Num. Sim. 56 (2018), 138-143.

14. M. Cadoni, R. Casadio, A. Giusti, W. Mück and M. Tuveri. Effective Fluid Description of the Dark Universe. Physics Letters B 776 (2018), 242-248.

15. A. Giusti. Dispersion relations for the time-fractional Cattaneo-Maxwell heat equation. J. Math. Phys. 59 (2018), 013506. 
16. R. Garra, A. Giusti and F. Mainardi. The fractional Dodson diffusion equation: a new approach. Ricerche di Matematica 67 (2018), 899-909.

17. A. Giusti. A comment on some new definitions of fractional derivative. Nonlinear Dynamics 93 (2018), 1757-1763.

18. R. Casadio, A. Giugno and A. Giusti. Corpuscular slow-roll inflation. Phys. Rev. D 97 (2018), 024041.

19. V. A. Diaz and A. Giusti. Fractional bosonic strings. J. Math. Phys. 59 (2018), 033509 .

20. A. Giugno, A. Giusti and A. Helou. Horizon quantum fuzziness for non-singular black holes. Eur. Phys. J. C 78 (2018), 208.

21. I. Colombaro, A. Giusti and S. Vitali. Storage and dissipation of energy in Prabhakar viscoelasticity. Mathematics 6 (2018), 15.

22. M. Cadoni, R. Casadio, A. Giusti and M. Tuveri. Emergence of a dark force in corpuscular gravity. Phys. Rev. D 97 (2018), 044047.

23. R. Casadio, A. Giugno, A. Giusti and O. Micu. Horizon quantum mechanics: spherically symmetric and rotating sources. Foundations of Physics 48 (2018), 1204-1218.

24. R. Casadio, A. Giusti and R. Rahim. Horizon quantum mechanics for spheroidal sources. Europhysics Letters 121 (2018), 60004.

25. I. Colombaro, R. Garra, A. Giusti and F. Mainardi. Scott-Blair models with timevarying viscosity. Applied Mathematics Letters 86 (2018), 57-63.

26. R. Casadio, A. Giusti and J. Mureika. Lower dimensional corpuscular gravity and the end of black hole evaporation. arXiv:1805.10444.

27. A. Giugno and A. Giusti. Domestic corpuscular inflaton. arXiv:1806.11168.

28. L. Frunzo, R. Garra, A. Giusti and V. Luongo. Modelling biological systems with an improved fractional Gompertz law. arXiv:1807.05937.

29. R. Casadio, A. Giugno, A. Giusti and M. Lenzi. Quantum Formation of Primordial Black holes. arXiv:1810.05185.

30. R. Rahim, A. Giusti and R. Casadio. The marginally trapped surfaces in spheroidal space-times. Int. J. Mod. Phys. D 28 (2019), 1950021. 


\title{
Chapter 2
}

\section{Semiclassical gravity and related issues}

\author{
Gravity slows down time. The closer \\ you are to the source, the slower time \\ will move. If you're standing in your \\ garden, you're head is travelling faster \\ through time than your feet. Don't \\ they teach you this stuff at space \\ school?
}

The Doctor

The aim of this Chapter is to review some general (well established) results of the semiclassical approach to the theory of gravitation. Given that the first rudimentary implementation of the corpuscular model of gravity was presented for black holes, in this Chapter we will focus mostly on this context by recalling known effects such as the one predicted by Hawking in the seventies [11. Nonetheless, one should not forget that the study of quantum effects on curved (classical) backgrounds has led to major implications for the modern description of the early Universe (see e.g. 221,23, 25, 28]).

\subsection{Black hole termodynamics and the need for a quan- tum theory}

The most evident hints, coming from GR, concerning the need for a quantum theory of gravitation are the so called laws of black hole mechanics [29].

First of all, it is worth recalling that these four laws tell us that the main features of black holes (of the Kerr-Newman class), at the dynamical level, are synthetized by seven quantities: the Arnowitt-Deser-Misner (ADM) mass $M$, the horizon's surface gravity $\kappa$, the horizon area $A$, the angular velocity $\Omega$, the (Komar) total angular momentum $J$, the electrostatic potential $\Phi$ and the electric charge $Q$. To be more precise, let us consider a 
stationary, analytic, asymptotically flat vacuum or electrovacuum ${ }^{1}$ black hole spacetime. For such a spacetime it is known (see [30|) that the (future) event horizon $\mathscr{H}^{+}$is a Killing horizon. Now, the relevance of the previous assumptions is due to the fact that they allow to relate the notion of event horizon to the one of Killing vector field, and thus to the concept of surface gravity. Indeed, if $\mathscr{K}$ is a Killing horizon defined by the vanishing of the norm of a certain Killing field $k$, then

$$
\left.\nabla_{k} k\right|_{\mathscr{K}}=\kappa k
$$

where $\kappa$ is the so called surface gravity of $\mathscr{K}$. Thus, by means of Raychaudhuri's equation, it is easy to prove the following statement

Theorem 1 (Zeroth law, [30]). Assuming the dominant energy condition for a stationary black hole spacetime, then the surface gravity is constant on $\mathscr{H}^{+}$.

Another interesting problem in classical black hole physics consist in understanding what happens if we slightly modify the parameters $(M, J, Q)$ for a black hole of the KerrNewman family. Naively, one would expect a variation in some of the remaining "main quantities" discussed above. Indeed, if we consider some linearized metric perturbations of the Kerr-Newman metric, namely $g(M+\delta M, J+\delta J, Q+\delta Q)-g(M, J, Q)$ for some small variations $\delta M, \delta J$ and $\delta Q$, one can prove that

Theorem 2 (First law, 29,31,32]). For any non-singular, asymptotically flat perturbation of a stationary, axisymmetric black hole with bifurcate horizon, we have that

$$
\frac{\kappa c^{2}}{8 \pi G_{\mathrm{N}}} \delta A=c^{2} \delta M-\Omega \delta J+\Phi \delta Q,
$$

where $G_{\mathrm{N}}$ is Newton's gravitational constant and c denotes the speed of light.

One the same line of thought, after analysing how a Kerr-Newman black hole reacts to a slight perturbation of $(M, J, Q)$, one could wonder what happens to these parameters if we throw in some matter, or if we let two black holes collide, once the systems has stabilized. The solution to this important conundrum was first provided by Hawking in 1972, through the following result

Theorem 3 (Second law - Hawking's area theorem, 29, 30, 33]). Let ( $\mathcal{M}, g$ ) a strongly asymptotically predictable spacetime satisfying the Einstein equation with a matter content satisfying the null energy condition. Let $\mathcal{U} \subset \mathcal{M}$ be a globally hyperbolic region for which $\overline{J^{-}\left(\mathscr{I}^{+}\right)} \subset \mathcal{U}$. Furthermore, let $\Sigma_{1}$ and $\Sigma_{2}$ be spacelike Cauchy surfaces for $U$ with $\Sigma_{2} \subset$ $J^{+}\left(\Sigma_{1}\right)$. Then, denoting by $H_{1} \equiv \mathscr{H}^{+} \cap \Sigma_{1}$ and $H_{2} \equiv \mathscr{H}^{+} \cap \Sigma_{2}$, we have that

$$
\operatorname{Area}\left(H_{2}\right) \geq \operatorname{Area}\left(H_{1}\right)
$$

Or, in a less frightening form

\footnotetext{
${ }^{1}$ Provided that the matter component is governed by hyperbolic equations.
} 
Proposition 1. The area $A$ of the event horizon of a black hole does not decrease with time, i.e. $\delta A \geq 0$.

A last interesting and rather useful result coming from black hole mechanics deals with the relation between the surface gravity of a black hole and reversible (and irreversible) transformations 29, 34]. Specifically one finds that

Theorem 4 (Third law, 29,35]). For a black hole, it is not possible to reduce $\kappa$ to zero by a finite sequence of operations.

It is worth remarking that, despite the fact that original formulation of the third law by Bardeen, Carter and Hawking [29] dates back to 1973, a precise mathematical formulation (and proof) of the latter was first presented, in 1986, by Israel in [35].

Let us now consider a charged thermodynamic system that undergoes an isochoric process. Such a process is governed by the first law of thermodynamics, that reads

$$
\mathrm{d} E=T \mathrm{~d} S+\mu \mathrm{d} N-\Phi \mathrm{d} Q,
$$

where $E$ represents the internal energy of the system, $T$ is the temperature, $S$ is the entropy of the system, $\mu$ is the chemical potential, and $N$ is the number of constituents. Moreover, we also known that if this system is isolated, then this process must increase the system's entropy. Besides, because of the third law of thermodynamics, there is no thermodynamic transformation that can reduce the entropy of a system to its absolute-zero value in a finite number of operations.

Form this very simple example one can immediately spot a strong similarity between the laws of black hole mechanics and the fundamental pillars of thermodynamics. Indeed, if we make the replacements:

$$
E=M c^{2}, \quad T=\alpha \kappa, \quad S=\beta \frac{A}{8 \pi}, \quad \mu=\eta \Omega, \quad N=\eta^{-1} J,
$$

for some dimensionful constants $\alpha, \beta, \eta$, we find a one to one map between the laws of black hole mechanics and the laws of thermodynamics [36]. This formal analogy suggests that black holes can be treated as thermodynamic objects. A stronger bound between black holes and thermodynamics, that goes beyond a mere formal analogy, is provided by Bekenstein's argument [37|. Indeed, let us assume that an observer decides to throw an object, carrying a certain amount of information, into a black hole. If the black hole carries no thermodynamic entropy, and the only feature of the latter are the one specified by the no-hair theorem (or uniqueness theorem) [38, 39], then, once the black hole has settled down to equilibrium, the entropy of the Universe would have decreased according to the observer, who remains outside the hole, potentially violating the second law of thermodynamics. This potential violation is related to the fact that the observer has no way to infer anything about the interior entropy of the black hole, since the two regions of the spacetime are causally disconnected. For this reason, Bekenstein in 1973 suggested that black holes had to be proper thermodynamic objects equipped with an entropy [40] 
proportional to their area, like in (2.4). This entropy function is now known in the literature as Bekenstein-Hawking entropy.

The only issue with this picture is that, if a black hole is a thermodynamics system, with a given entropy and temperature, then it must emit radiation. But this is inconsistent with the classical notion of black hole. This issue is somehow resolved by considering quantum mechanical matter fields propagating on a classical black hole background. This line of thought is actually what led to the theoretical discovery of the Hawking effect [11], in 1974.

\subsection{Quantum fields on black hole spacetimes}

As discussed in Section 2.1, Bekenstein's entropic picture for black holes begs for a quantum mechanical description of some aspects of Einstein's gravity. Quantum field theory (QFT) on curved spacetimes [41, 42 provides a rigorous mathematical framework for studying quantum matter fields propagating on classical backgrounds, provided that one works in a setting for which purely quantum gravitational effects can be neglected.

The fundamental length scale for quantum gravity is usually set to the Planck length $\ell_{\mathrm{P}}=\sqrt{\hbar G_{\mathrm{N}} / c^{3}} \sim 10^{-35} \mathrm{~m}$ or, as an energy scale, to the Planck mass $m_{\mathrm{P}}=\sqrt{\hbar c / G_{\mathrm{N}}} \sim$ $10^{19} \mathrm{GeV} / \mathrm{c}^{2}$. This scale can be understood as a minimum localization scale, indeed if we wish to localize a quantum particle in a region $\Delta x \lesssim \ell_{\mathrm{P}}$ we have to equip it with an energy $\Delta E \gtrsim \hbar c / \Delta x$, because of Heisenberg's uncertainty principle. Then the gravitational radius for the particle would exceed the size of the particle itself, i.e. $R_{\mathrm{H}}=2 G_{\mathrm{N}} \Delta E / c^{2} \gtrsim$ $\ell_{\mathrm{P}}^{2} / \Delta x \gtrsim \Delta x$, signaling that gravity prevents us from being able to resolve scales shorter than the Planck length. One can also provide a sort of quantum field theoretic justification for identifying the Planck length as the scale of quantum gravity. Indeed, if we consider pure Einstein's gravity as an effective (classical) field theory of gravitation, we have that the Einstein-Hilbert action, i.e.

$$
S_{\mathrm{EH}}=-\frac{c^{4}}{16 \pi G_{\mathrm{N}}} \int \mathrm{d}^{4} x \sqrt{-\operatorname{det} g} R=-\frac{c \hbar}{16 \pi \ell_{\mathrm{P}}^{2}} \int \mathrm{d}^{4} x \sqrt{-\operatorname{det} g} R
$$

with $R$ representing the Ricci scalar, plays the role of an "effective action" for the gravitational field. If we now consider a spacetime with curvature radius $L$ (i.e. $R \sim L^{-2}$ ) and four volume $L^{4}$, then $S_{\mathrm{EH}}(L) \sim \hbar\left(L / \ell_{\mathrm{P}}\right)^{2}$ that suggests that quantum curvature fluctuations are substantially suppressed if $L>\ell_{\mathrm{P}}$, whereas they would pile up and give non-negligible contributions for $L \lesssim \ell_{\mathrm{P}}$. When studying quantum field theory on curved backgrounds one then have to assume to be working in a regime which is fairly far from the Planck scale. This means that we have to keep ourselves away from high curvature regions of the spacetime, such as regions near black hole singularities.

Given these premises, we can now enter more deeply into the key feature of QFT on curved spacetime. The main idea of this approach is to try to put together the fundamental principles of the general theory of relativity (GR) and quantum field theory (on Minkowski space) in the most coherent way possible. 
As far as GR is concerned, it is rather easy to spot on which basic principle it is based. Indeed, the two key feature of GR are the assumption the a spacetime is a Lorentzian manifold $(\mathcal{M}, g)$, with $g$ being a metric with Lorentz signature, and that both the metric and matter are dynamical objects, whose evolution is locally determined by Einstein's field equations. The last requirement can be rephrased in a more rigorous form by demanding a well-posed initial value problem for the field equations.

Finding the fundamental bricks on which QFT is built is, however, a bit trickier (see [41]). Therefore we will not attempt to provide the most general description of the fundamental feature of QFT on the Minkowski space, but rather we will try to identify some minimal principles that can be consistently generalized to curved spaces.

First of all, as we shall see later on, the notions of particle and vacuum state have almost no meaning for general spacetimes, so we shall abandon them as key concepts. Besides, also the Poincaré invariance presents some problems for general spacetime, but it still provides a major requirement at the local level.

For sure, an idea that can be easily carried along to general spacetimes is that the fundamental constituents of matter are represented by tensor fields. In the flat case these fields are infinite dimensional representations of the Lorentz group, however this idea must make sense only at the local level when considering general spaces. Another key principle of QFT on Minkowski is that quantum fields are operator-valued distributions that have to satisfy some commutation relations. Thus we can take as a basic principle that these fields have to be regarded as operator-valued distributions in an algebra. Finally, quantum fields are usually supposed to satisfy some microlocal conditions (see [41]), such as having an hamiltonian bounded from below.

All that being said, let us focus our attention on the case of a massless scalar field $\Phi(x)$ on a classical background spacetime with a metric $g_{\mu \nu}(x)$. Let us then consider a spacetime $(\mathcal{M}, g)$ which is globally hyperbolic and that can be foliated (ADM three-plusone decomposition [43|) by "fixed-time" Cauchy surfaces $\Sigma_{t}=\{t=$ const. $\}$. The action for a massless real Klein-Gordon field on $(\mathcal{M}, g)$ reads

$$
S_{\Phi}=-\frac{1}{2} \int_{\mathcal{M}} \mathrm{d}^{4} x \sqrt{-\operatorname{det} g} g^{\mu \nu} \partial_{\mu} \Phi \partial_{\nu} \Phi,
$$

whose equations of motion will then read

$$
g^{\mu \nu} \nabla_{\mu} \nabla_{\nu} \Phi=0,
$$

where $\nabla$ denotes the covariant derivative and $[\Phi]=$ energy $^{1 / 2}$ length $^{-1 / 2}$. In order to continue with the quantization of the free real scalar field we have to compute the conjugate momentum $\Pi(x)$ to the field $\Phi(x)$, which is defined by

$$
\Pi(x) \equiv \frac{\delta S_{\Phi}}{\delta\left(\partial_{0} \Phi(x)\right)}=-\sqrt{-\operatorname{det} g} g^{0 \mu} \partial_{\mu} \Phi(x),
$$

then we impose the (equal time) canonical commutation relations, i.e.

$$
\left[\Phi(t, \boldsymbol{x}), \Pi\left(t, \boldsymbol{x}^{\prime}\right)\right]=i \hbar \delta^{(3)}\left(\boldsymbol{x}-\boldsymbol{x}^{\prime}\right), \quad\left[\Phi(t, \boldsymbol{x}), \Phi\left(t, \boldsymbol{x}^{\prime}\right)\right]=\left[\Pi(t, \boldsymbol{x}), \Pi\left(t, \boldsymbol{x}^{\prime}\right)\right]=0 .
$$


Given this setting, we shall now try to build the Hilbert space on which these operators act. If we denote $\mathcal{A}$ is the space of complex solutions of $(2.8)$, then $\forall f_{1}, f_{2} \in \mathcal{A}$ we can define a scalar product

$$
\left(f_{1}, f_{2}\right)=\frac{i}{\hbar} \int_{\Sigma_{0}} \mathrm{~d}^{3} x \sqrt{\operatorname{det}^{(3)} g} n^{\mu}\left(f_{1}^{*} \partial_{\mu} f_{2}-f_{2}^{*} \partial_{\mu} f_{1}\right),
$$

for some $\Sigma_{0}$ with normal vector $n$ and induced metric ${ }^{(3)} g$. Furthermore, if we define a current $J^{\mu}:=-(i / \hbar)\left(f_{1}^{*} \partial^{\mu} f_{2}-f_{2}^{*} \partial^{\mu} f_{1}\right)$ one can easily show that $\nabla^{\mu} J_{\mu}=0$, i.e. $J^{\mu}$ is conserved. This conservation property makes the scalar product 2.11 independent of the choice of $\Sigma_{0}$, that can now be replaced by any constant time Cauchy surface $\Sigma_{t}$. It is also easy to see that 2.11) is an hermitian non-degenerate form, although it is not positive, in general.

One of the main issues for general spacetimes is that, unless we restrict ourselves to stationary cases, we do not have a preferred choice for the decomposition of $\mathcal{A}$ into solutions with positive and negative frequencies, i.e. for which the squared norm induced by (2.11) is, respectively, positively or negatively defined. Once we make the choice for the space of positive frequency solutions of (2.8), let us call it $\mathcal{P}$, we can immediately make the decomposition $\mathcal{A}=\mathcal{P} \oplus \mathcal{N}$, with $\mathcal{N}$ being the corresponding space of negative frequency solutions of (2.8). Given this decomposition one can define the creation and annihilation operators associated to $f \in \mathcal{P}$ as

$$
a(f)^{\dagger}:=-\left(f^{*}, \Phi\right), \quad a(f):=(f, \Phi)
$$

then, from the canonical commutation relations we immediately infer that

$$
\left[a\left(f_{1}\right), a\left(f_{2}\right)^{\dagger}\right]=\left(f_{1}, f_{2}\right), \quad\left[a\left(f_{1}\right), a\left(f_{2}\right)\right]=0, \quad\left[a\left(f_{1}\right)^{\dagger}, a\left(f_{2}\right)^{\dagger}\right]=0,
$$

for all $f_{1}, f_{2} \in \mathcal{P}$. We can then define the vacuum state corresponding to our choice of $\mathcal{P}$, i.e. $\left|0_{\mathcal{P}}\right\rangle$, as

$$
a(f)\left|0_{\mathcal{P}}\right\rangle=0, \quad \forall f \in \mathcal{P},
$$

together with the normalization $\left\langle_{\mathcal{P}} 0 \mid 0_{\mathcal{P}}\right\rangle=1$.

At this point the construction of the $N$-particle Fock space is rather straightforward, indeed if $f_{1}, \ldots, f_{N} \in \mathcal{P}$ are $N$ positive frequency "modes", then the corresponding $N$ particle Fock state is given by $\left|f_{1}, \ldots, f_{N}\right\rangle_{\mathcal{P}} \propto a\left(f_{1}\right) \cdots a\left(f_{N}\right)\left|0_{\mathcal{P}}\right\rangle$, up to normalization. Of course, if we now choose a different space of positive frequency solutions, $\left|0_{\mathcal{P}}\right\rangle$ will not be the vacuum for this new "mode decomposition". Moreover, the new vacuum state will not even belong to the same Hilbert space defined starting from $\mathcal{P}$. This fact is actually one of the reasons that leads to the particle production in non-stationary spacetimes.

To see the particle creation effect we first have to see how different choices for the decomposition of $\mathcal{A}$ are related. Thus, let us consider two distinct choices for the space of positive frequency solutions and denote them by $\mathcal{P}$ and $\mathcal{P}^{\prime}$. Now, on each space we can 
choose an orthonormal basis ${ }^{2}$, say $\left\{f_{i}\right\}$ and $\left\{f^{\prime}{ }_{i}\right\}$ respectively, for which we have the two decomposition of the real scalar field,

$$
\Phi=\sum_{k}\left[f_{k} a\left(f_{k}\right)+f_{k}^{*} a\left(f_{k}\right)^{\dagger}\right], \quad \Phi=\sum_{k}\left[f_{k}^{\prime} a\left(f_{k}^{\prime}\right)+f_{k}^{\prime *} a\left(f_{k}^{\prime}\right)^{\dagger}\right]
$$

These tow basis are relate to one another through the so called Bogoliubov transformation, i.e.

$$
f_{i}^{\prime}=\sum_{j}\left[\alpha_{i j} f_{j}+\beta_{i j} f_{j}^{*}\right]
$$

which also tell us how the creation and annihilation operators transform when switching from $\mathcal{P}$ to $\mathcal{P}^{\prime}$, i.e.

$$
a\left(f_{i}^{\prime}\right)=\sum_{j}\left[\alpha_{i j}^{*} a\left(f_{j}\right)-\beta_{i j}^{*} a\left(f_{j}\right)^{\dagger}\right] .
$$

Let us now consider a spacetime $\mathcal{M}$ that starts in a stationary phase $\mathcal{M}_{\text {in }}$, undergoes a non-stationary regime $\mathcal{M}_{\mathrm{NS}}$ and ultimately settles down to a new stationary phase $\mathcal{M}_{\text {out }}$, i.e. $\mathcal{M}=\mathcal{M}_{\text {in }} \cup \mathcal{M}_{\mathrm{NS}} \cup \mathcal{M}_{\text {out }}$. Such a behavior can potentially be realized (ideally) by several different physical settings. An example could be given by a particle (or a planet) falling into a Schwarzschild black hole. Indeed, when they are far away from one another the spacetime is substantially stationary then, when the hole swallows the particle, the whole system goes through a non-stationary phase that stabilizes, after some time, to a Schwarzschild spacetime with an increased mass. The assumption of global hyperbolicity tell us that any solution of (2.8) in any of the two patches $\mathcal{M}_{\text {in/out }}$ can be uniquely extended to the whole spacetime. This signals that we have two possible choices for the state of positive frequency solutions of (2.8), due to the fact that $\mathcal{M}_{\text {in/out }}$ are stationary and therefore we have a preferred way to choose $\mathcal{P}_{\text {in }}$ and $\mathcal{P}_{\text {out }}$.

As argued above, in general the two vacuum states $\left|0_{\mathcal{P}_{\text {in }}}\right\rangle$ and $\left|0_{\mathcal{P}_{\text {out }}}\right\rangle$ will differ, and they might not even belong to the same Hilbert space. If one now assume that the initial state of the system does not contain any modes, i.e. $|i n\rangle=\left|0_{\mathcal{P}_{i n}}\right\rangle$, it is rather instructive to see how many "late-time" modes can be found in $|i n\rangle$. Considering a mode $f_{i}^{\text {out }}$ of the basis of $\mathcal{P}_{\text {out }}$ and denoting the number operator of the "out modes" by $\widehat{N}\left(f_{i}^{\text {out }}\right) \equiv a\left(f_{i}^{\text {out }}\right)^{\dagger} a\left(f_{i}^{\text {out }}\right)$, by means of the Bogoliubov transformation one finds

$$
\left\langle\mathcal{P}_{\text {in }} 0\left|\widehat{N}\left(f_{i}^{\text {out }}\right)\right| 0_{\mathcal{P}_{\text {in }}}\right\rangle=\sum_{j}\left|\beta_{i j}\right|^{2},
$$

so, if $\widehat{N}^{\text {out }}=\sum_{i} \widehat{N}\left(f_{i}^{\text {out }}\right)$ then

$$
\left\langle\mathcal{P}_{\text {in }} 0\left|\widehat{N}^{\text {out }}\right| 0_{\mathcal{P}_{\text {in }}}\right\rangle=\operatorname{Tr}\left(\beta \beta^{\dagger}\right),
$$

\footnotetext{
${ }^{2}$ Which implies that $\left[a\left(f_{h}\right), a\left(f_{k}\right)^{\dagger}\right]=\delta_{h k}$.
} 
that proves the particle creation effect [11,44 for non-stationary spacetimes, as anticipated.

Following a similar line of thought, in 1974 Hawking [11] was able to prove that black holes are (theoretically) not inert objects, but rather they emit thermal radiation known as Hawking radiation. Originally Hawking considered the propagation of massless free real scalar field on a classical dynamical background provided by a gravitational collapse to form a Schwarzschild black hole. Assuming that the initial state of the system was prepared in such a way that the scalar field was initially in its vacuum state, he then computed the number of particles at infinity measured at late times. Specifically, Hawking found that black hole radiates to infinity with a spectrum given by

$$
\left\langle\mathcal{P}_{\text {in }} 0\left|\widehat{N}^{\text {out }}\right| 0_{\mathcal{P}_{\text {in }}}\right\rangle=\sum_{i} \frac{\Gamma_{i}}{\exp \left(2 \pi \epsilon_{i} / \hbar c \kappa\right)-1},
$$

where $\Gamma_{i}$ is the so-called greybody factor that measures the fraction of a mode of frequency $\epsilon_{i} / \hbar$ that would be absorbed by the black hole.

From 2.19 one can infer several interesting things. First of all, one can easily deduce that a black hole emits black body radiation at a temperature, called Hawking temperature,

$$
T_{\mathrm{H}}=\frac{\hbar c \kappa}{2 \pi k_{\mathrm{B}}},
$$

with $k_{\mathrm{B}}$ being the Boltzmann constant and $\kappa$ denoting the surface gravity of the black hole. Secondly, 2.20 allows us to properly fix the proportionality coefficients in (2.5), which means that we can uniquely identify the Bekenstein-Hawking entropy with

$$
S_{\mathrm{BH}}=\frac{k_{\mathrm{B}} A}{4 \ell_{\mathrm{P}}^{2}} .
$$

Furthermore, if one considers the case of a Schwarzschild black hole, it is easy to see that $\kappa=c^{2} / 4 G_{\mathrm{N}} M$ and thus $T_{\mathrm{H}}=\hbar c^{3} / 8 \pi k_{\mathrm{B}} G_{\mathrm{N}} M$. In this case the first law of black hole thermodynamics reduces to $c^{2} \mathrm{~d} M=T_{\mathrm{H}} \mathrm{d} S_{\mathrm{BH}}$, that can be used to explicitly compute the heat capacity for a Schwarzschild black hole, i.e.

$$
C_{V}=\frac{\mathrm{d} E}{\mathrm{~d} T}=c^{2}\left(\frac{\mathrm{d} T_{\mathrm{H}}(M)}{\mathrm{d} M}\right)^{-1}=-\frac{8 \pi k_{\mathrm{B}} G_{\mathrm{N}}}{c \hbar} M^{2}<0,
$$

thus, black holes have negative heat capacity.

Despite this extreme coherence between the results for black hole thermodynamics and quantum field theoretic computations on curved backgrounds, there is a nasty issue hiding in the dark. Indeed, let us recall the Stefan-Boltzmann law, which states that the total energy radiated per unit surface area of a black body, across all frequencies, per unit time is proportional to the fourth power of the black body's temperature. Then, for a Schwarzschild black hole we have

$$
\frac{\mathrm{d} E}{\mathrm{~d} t}=-\sigma A_{\mathrm{H}} T_{\mathrm{H}}^{4}
$$


with $\sigma$ denoting the Stefan-Boltzmann constant and $A_{\mathrm{H}}$ representing the area of the horizon. Hence, recalling that $E=M c^{2}, A_{\mathrm{H}}=4 \pi R_{\mathrm{H}}^{2}$ and that $T_{\mathrm{H}}=\hbar c^{3} / 8 \pi k_{\mathrm{B}} G_{\mathrm{N}} M$ one can easily infer that

$$
\frac{\mathrm{d} M}{\mathrm{~d} t}=-\frac{\hbar c^{4}}{15360 \pi G_{\mathrm{N}}^{2} M^{2}},
$$

which tells us that the energy carried by the Hawking radiation is extracted from the black hole itself and, thus, that the black hole slowly evaporates away because of the Hawking effect. Furthermore, one can even estimate the time that would be needed for the black hole to evaporate completely (provided that we neglect the backreaction of the spacetime to the radiation), indeed if we integrate the latter equation we get

$$
\tau=\frac{5120 \pi G_{\mathrm{N}}^{2}}{\hbar c^{4}} M_{\text {initial }}^{3}
$$

However, the idea that a black hole can potentially evaporate completely poses a serious problem form a quantum mechanical perspective. Indeed, let us assume that we prepare some distribution of matter in a pure state. Then we let this matter collapse to form a black hole. If we trust Hawking's model all the way through the complete evaporation of the black hole, at the end of the process we would be left only with thermal radiation in our system, which is a mixed state. But this would violate a fundamental principle of quantum mechanics, namely the requirement for a unitary evolution of quantum states. This issue is what is known in the literature as information paradox [45-48].

It is now worth remarking that the second law of black hole thermodynamics tells us that the Bekenstein-Hawking entropy must not decrease classically, however it can decrease quantum mechanically via the Hawking evaporation process. Nonetheless, thermal radiation itself carries an entropy, so the total entropy (radiation + Bekenstein-Hawking) does not decrease. These remarks lead to the so called generalized second law of black hole thermodynamics [40], which states that the total entropy (black hole + matter) must not decrease in any physical process. If we try to interpret the Bekenstein-Hawking entropy in statistical terms, one can easily compute the corresponding number of microstates which are responsible for such an entropy, i.e. $N=\exp \left(S_{\mathrm{BH}} / k_{\mathrm{B}}\right)$. A rather common view depicts these microstates as localized near the black hole horizon, which suggests an interpretation of the entropy-area relation as an hint to the fact that the information contained in a black hole is substantially stored on its surface, rather than in its causally inaccessible interior (see e.g. [49,50]). In this statistical perspective, the notion of entanglement entropy plays also a key role. One of the main results coming from these ideas is that the "black hole subsystem" is maximally entangled with the "radiation subsystem" 48,51. Nonetheless, half way thorough the process of evaporation (Page time, time when a black hole has half of its initial entropy) a major issue emerges. Indeed, continuing with the evaporation the black hole would keep piling up entanglement entropy by this process exceeding the Bekenstein entropy bound [52,53. If one then tries to prevent this issue by assuming the radiation subsystems, at early and late times, to be maximally entangled, then this forces 
bipartite entanglements to evolve into tripartite states. This is, however, not possible if we assume a unitary time evolution for the system. Hence, the idea is that when the Page time is reached either the assumption of unitary evolution or the equivalence principle should fail.

\subsection{Remarks on gravity as a quantum field theory}

In Section 2.2 we have discussed what happens if we quantize matter fields on a curved classical background. In this section, instead, we will try to provide a bottom-up picture of gravity based on a quantum field theoretic perspective. To be more precise, we will try to describe gravity as a QFT of Spin-2 fields, the gravitons, working in the weak-field limit (or, in other words, in the weak gravity regime). As it will appear clear later on, this picture presents several issues, the most important of which is the fact that Einstein's gravity turns out to be non-renormalizable [54 56]. Just as a general remark, the following discussion will be based mostly on Donoghue's seminal works $[57,59$. . Besides, from now on we will set the value of the speed of light $c=1$, for sake of simplicity.

Let us assume to be interested in building a theory of gravity, without referring to general relativity at any time. Our first goal would be to find the appropriate field that allows to reproduce the experimentally observed gravitational effects.

First of all, one would be interested in recovering the Newtonian potential

$$
V_{\mathrm{N}}(r)=-G_{\mathrm{N}} \frac{m_{1} m_{2}}{r}
$$

given two masses $m_{1}$ and $m_{2}$, from the non-relativistic limit of such a theory, since it seems to work quite well if we wish to model most of the feature of the Solar system. Now, Newton's potential clearly resembles the Coulomb one. However, the gravitational potential is always attractive, whereas the Coulomb potential can be either attractive or repulsive. The latter is actually a feature of electrodynamics, that tells us that Spin1 fields, like the photon, cannot represent an appropriate mediator for the gravitational interaction. Hence, we are left to see which field between the Spin-0 and Spin-2 fits best for our task, since higher spin fields are difficult to include in the QFT picture in a consistent way. If we consider the Spin-0 case, one can easily spot some reasons for concern. Indeed, it is rather apparent that the source of the gravitational field should be the total energy density, and thus by the stress-energy tensor $T_{\mu \nu}$. Therefore, in a scalar field theory, the only allowed coupling would be given by $\mathcal{L}_{\text {int }} \sim \varphi T_{\mu}^{\mu}$. However, inspecting the case of an electromagnetic field as a source we have,

$$
T^{\mu \nu}=\frac{1}{\mu_{0}}\left[F^{\mu \alpha} F^{\nu}{ }_{\alpha}-\frac{1}{4} \eta^{\mu \nu} F_{\alpha \beta} F^{\alpha \beta}\right],
$$

with $\mu_{0}$ denoting the vacuum permeability and $F_{\mu \nu}$ representing the electromagnetic field strength, form which one cane easily conclude that $T_{\mu}^{\mu}=0$. Thus, such a coupling would immediately violate the equivalence principle, since there would be no interaction between 
light and gravity. Hence, the only option that remains available, within QFT, is the Spin-2 field.

Now, let us try to quantize the theory of a Spin-2 field emerging from the weak-field limit of the Einstein-Hilbert action 2.6). So assuming the ansatz $g_{\mu \nu}=\eta_{\mu \nu}+h_{\mu \nu}$, with $\eta_{\mu \nu}$ denoting the flat metric and $\left|h_{\mu \nu}\right| \ll 1$, then (2.6) can be rewritten as

$$
\begin{aligned}
S_{\mathrm{EH}} & =-\frac{1}{2 \chi} \int \mathrm{d}^{4} x \sqrt{-\operatorname{det} g} R= \\
& \simeq-\frac{1}{4 \chi} \int \mathrm{d}^{4} x\left(\frac{1}{2} \partial_{\mu} h_{\nu \rho} \partial^{\mu} h^{\nu \rho}-\partial_{\mu} h_{\nu \rho} \partial^{\nu} h^{\mu \rho}+\partial_{\mu} h \partial_{\rho} h^{\mu \rho}-\frac{1}{2} \partial_{\mu} h \partial^{\mu} h\right)
\end{aligned}
$$

where $\chi \equiv 8 \pi G_{\mathrm{N}}$ and $h \equiv \eta^{\mu \nu} h_{\mu \nu}$. Form the latter one can immediately recognize the massless Fierz-Pauli lagrangian 60,61, i.e.

$$
\mathcal{L}_{\mathrm{FP}}=-\frac{1}{2} \partial_{\mu} h_{\nu \rho} \partial^{\mu} h^{\nu \rho}+\partial_{\mu} h_{\nu \rho} \partial^{\nu} h^{\mu \rho}-\partial_{\mu} h \partial_{\rho} h^{\mu \rho}+\frac{1}{2} \partial_{\mu} h \partial^{\mu} h .
$$

Thus, computing the variation of the action 2.28 with respect to $h_{\mu \nu}$ one finds,

$$
\begin{aligned}
G_{\mu \nu} & \simeq \frac{1}{2} \varepsilon_{\mu \nu \alpha \beta} h^{\alpha \beta} \\
& \equiv \frac{1}{2}\left(-\square h_{\mu \nu}+\eta_{\mu \nu} \square h+\partial_{\mu} \partial^{\lambda} h_{\lambda \nu}+\partial_{\nu} \partial^{\lambda} h_{\lambda \mu}-\eta_{\mu \nu} \partial^{\lambda} \partial^{\rho} h_{\lambda \rho}-\partial_{\mu} \partial_{\nu} h\right) \\
& =0,
\end{aligned}
$$

or, starting from the Fierz-Pauli lagrangian (2.29), simply $\mathcal{E}_{\mu \nu \alpha \beta} h^{\alpha \beta}=0$.

Clearly, the decomposition $g_{\mu \nu}=\eta_{\mu \nu}+h_{\mu \nu}$ is not unique because of the diffeoinvariance of the theory. Here we are restricting ourselves to the class of diffeomorphisms that preserves the condition $\left|h_{\mu \nu}\right| \ll 1$, nonetheless the set of equivalent decompositions of this kind is still pretty large. Thus, in order to proceed with the quantization one have to fix the gauge, e.g. let us consider the harmonic (or de Donder) gauge $g^{\mu \nu} \Gamma_{\mu \nu}^{\alpha}=0$ or equivalently $\partial_{\mu} h_{\nu}^{\mu}-(1 / 2) \partial_{\nu} h=0$. Now, once the gauge has been fixed, the equations of motion for the free graviton 2.30 read

$$
\square h_{\mu \nu}=0 .
$$

The latter has traveling-wave solutions with four-momentum $p^{2}=0$, i.e.

$$
h_{\mu \nu} \sim \epsilon_{\mu \nu}(\boldsymbol{p}) e^{ \pm i p \cdot x / \hbar},
$$

where $p \cdot x \equiv p^{\mu} x_{\mu}=-E_{\boldsymbol{p}} t+\boldsymbol{p} \cdot \boldsymbol{x}$, with $\boldsymbol{p} \cdot \boldsymbol{x}$ denoting the Euclidean scalar product on $\mathbb{R}^{3}$ and with $\epsilon_{\mu \nu}(\boldsymbol{p})=\epsilon_{\nu \mu}(\boldsymbol{p})$ denoting the polarization tensor.

Despite the assumption of the de Donder gauge, we have not yet been able to completely fix the redundancy of our description of the free graviton. Indeed, if we make a coordinate transformation such that $x^{\mu} \rightarrow x^{\mu}+\xi^{\mu}(x)$, with $\xi^{\mu}(x)$ such that $\square \xi_{\mu}=0$. Thus, it turns out that we can chose $\xi_{\mu}$ in such a way that $h^{0 \mu}=0, h_{i}^{i}=0$ and $\partial^{i} h_{i j}=0$ completely 
fixing the gauge, from which we get some restrictions on the polarization tensor, namely $\epsilon_{0 \mu}=0, \epsilon_{i}^{i}=0$ and $p^{i} \epsilon_{i j}=0$. Since the free graviton has two helicity states one has that $\epsilon_{\mu \nu}(\boldsymbol{p})$ is replaced by $\epsilon_{\mu \nu}(\boldsymbol{p}, \lambda)$ with $\lambda= \pm$, that keeps track of the helicity, with the normalization $\epsilon_{\mu \nu}(\boldsymbol{p}, \lambda) \epsilon^{\mu \nu}\left(\boldsymbol{p}, \lambda^{\prime}\right)=\delta_{\lambda \lambda^{\prime}}$. Now, the general plane-wave expansion for $h_{\mu \nu}$ reads

$$
h_{\mu \nu}=\sum_{\lambda=+,-} \int \mathrm{d} \mu(\boldsymbol{p})\left[a(\boldsymbol{p}, \lambda) \epsilon_{\mu \nu}(\boldsymbol{p}, \lambda) e^{i p \cdot x / \hbar}+\mathrm{h} . \mathrm{c} .\right] .
$$

Remark. It is important to observe that we are employing the mostly plus convention for the Lorentz signature, i.e. $(-+++)$, that implies $\eta_{\mu \nu} \equiv \operatorname{diag}(-1,1,1,1)$.

At this point, we have to promote $a(\boldsymbol{p}, \lambda)$ and $a(\boldsymbol{p}, \lambda)^{*}$ to distribution-valued operators $a(\boldsymbol{p}, \lambda)$ and $a(\boldsymbol{p}, \lambda)^{\dagger}$ with canonical commutation relations

$$
\left[a(\boldsymbol{p}, \lambda), a\left(\boldsymbol{q}, \lambda^{\prime}\right)^{\dagger}\right]=\delta^{(3)}(\boldsymbol{p}-\boldsymbol{q}) \delta_{\lambda \lambda^{\prime}} .
$$

In order to compute the Feynman propagator for the graviton one has to invert the kinetic term of the action 2.29 , that gives (in the momentum space)

$$
i D_{\alpha \beta \mu \nu}^{(F)}(p)=\frac{i P_{\alpha \beta \mu \nu}}{p^{2}-i \varepsilon}
$$

with $P_{\alpha \beta \mu \nu}$ denoting the projection operator, defined as

$$
P_{\alpha \beta \mu \nu} \equiv \sum_{\lambda} \epsilon_{\mu \nu}(\boldsymbol{p}, \lambda) \epsilon_{\mu \nu}^{*}(\boldsymbol{p}, \lambda)=\frac{1}{2}\left(\eta_{\alpha \mu} \eta_{\beta \nu}+\eta_{\alpha \nu} \eta_{\beta \mu}-\eta_{\alpha \beta} \eta_{\mu \nu}\right) \text {. }
$$

Given all these premises, we can now introduce an interaction between a massive scalar field $\Phi$ and the graviton. As we said before, the graviton should interact with matter fields through their stress-energy tensor, thus recalling that

$$
T_{\mu \nu}^{(\Phi)}=\partial_{\mu} \Phi \partial_{\nu} \Phi-\frac{1}{2} \eta_{\mu \nu}\left(\partial^{\lambda} \Phi \partial_{\lambda} \Phi+m_{\Phi}^{2} \Phi^{2}\right)
$$

one can introduce the coupling with matter in the action as

$$
S=\int \mathrm{d}^{4} x\left(\frac{1}{4 \chi} \mathcal{L}_{\mathrm{FP}}+\frac{1}{2} h^{\mu \nu} T_{\mu \nu}^{(\Phi)}\right)
$$

From this action one can then read off the Feynman rules for the graviton, see e.g. 62 65.

It is interesting to compute the amplitude for the scattering of two scalars mediated by a graviton at the tree-level, namely the amplitude for the Feynman graph in Figure 2.1. 


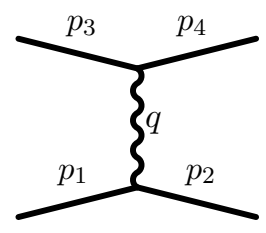

Figure 2.1: Tree-level scattering of two $\Phi$ fields of mass $m$ mediated by a graviton.

Specifically, in the non-relativistic limit $p^{\mu} \sim(m, \mathbf{0})$, one finds that this amplitude reads

$$
\mathcal{M}=-\chi \frac{m^{2}}{q^{2}},
$$

that, thanks to the Born approximation and recalling that $\chi=8 \pi G_{\mathrm{N}}$, once we invert back to $r$-space we find

$$
V(r)=-G_{\mathrm{N}} \frac{m^{2}}{r}
$$

which is exactly Newton's law, see e.g. [66]. However, this approach has a major issue. If we consider a loop-diagrams like the one in Figure 2.2, such diagrams cannot be renormalized within the Einstein-Hilbert framework, since it would require terms with four derivative of $h_{\mu \nu}$ in the action, see e.g. [54 [56, 66]. Nonetheless, if we focus on the case of pure gravity $\left(T_{\mu \nu}=0\right)$ one can find that this theory is finite at one loop, even thought this holds only in four dimensions because of the Gauss-Bonnet term 67

$$
\sqrt{-\operatorname{det} g} \mathcal{G}=\sqrt{-\operatorname{det} g}\left(R_{\alpha \beta \mu \nu} R^{\alpha \beta \mu \nu}-4 R_{\alpha \beta} R^{\alpha \beta}+R^{2}\right)
$$

which can be recast as a four divergence of a topological current and serve the purpose of restoring the one-loop finiteness of gravity without affecting the equations of motion. However, as shown by Goroff and Sagnotti in [55], if we proceed with two-loops computations we find that, even for pure gravity in four dimensions, the emerging divergent graphs cannot be cured by means of the renormalization of the Einstein-Hilbert action. This can be summarized by saying that Einstein's gravity is a non-renormalizable theory.

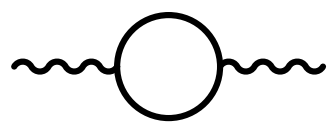

Figure 2.2: $\Phi$-loop in the graviton propagator. 


\title{
Chapter 3
}

\section{Alternative perspectives on quantum effects in gravity}

\author{
Che cos'è il genio? È fantasia, \\ intuizione, decisione e velocità \\ d'esecuzione.
}

Il Perozzi

As discussed in Chapter 2, semiclassical gravity is commonly understood as the study of the dynamics of quantum fields as they propagates on classical curved backgrounds, assuming that the contribution of the backreaction is negligible. Besides, gravity itself has a quantum field theoretic description in terms of massless Spin-2 tensor fields on the Minkowski space, in the weak-field limit. Both these approaches provide an insightful view on quantum mechanical effects in gravity, however they also predict some serious conceptual issues: the information loss paradox and non-renormalizability.

Now, it is important to remark that, from a purely quantum field theoretic perspective, the non-renormalizability of gravity does not represent a massive problem for the theory per se. Indeed, if one follows the Wilsonian viewpoint, it is easy to conclude that a nonrenormalizable theory, like gravity, is still a perfectly fine low-energy effective field theory, provided that we keep away from its cut-off scale $\Lambda \sim m_{\mathrm{P}}$. Indeed, computing $N$-point amplitudes for gravity (in the weak field limit) [57 one finds

$$
\mathcal{M}_{N}=\mathcal{M}_{N}^{(0)}(E)\left[1+c_{1} \frac{E^{2}}{m_{\mathrm{P}}^{2}}+c_{2} \frac{E^{2}}{m_{\mathrm{P}}^{2}} \log \left(\frac{E^{2}}{m_{\mathrm{P}}^{2}}\right)+\cdots\right]
$$

where $\mathcal{M}_{N}^{(0)}(E)$ is the bare $N$-point amplitude and $E$ is the energy scale of the scattering process. Clearly, this theory seems to work rather well provided that $E \ll m_{\mathrm{P}}$, and it even allows to compute some explicit quantum corrections to classical spacetimes, see e.g. [57, 58, 68. There are, however, some conceptual issues with the extreme low-energy limit of this approach, since gravitational effects may build up to the point that the integrated 
curvature may be large even in regions where the local curvature is not. We invite the interested reader to refer to [57,69].

In this section we will review two approaches to quantum effects in gravity which offer an alternative perspective on the matters presented in Chapter 2 .

\subsection{Horizon quantum mechanics}

The aim of this section is to review the main features of the horizon quantum mechanics (HQM) formalism, paying particular attention to static spherically symmetric and rotating spacetimes. As a general information, it is important to remark that the discussion presented here is based mostly on $[70-84]$, thus we invite the interested reader to refer to these articles for further details on the formalism.

\subsubsection{Trapped surfaces and event horizons in general relativity}

One of the key prediction of GR is the existence of objects for which the curvature of the spacetime that surrounds them is so high that even the light is unable to escape their gravitational pull. These exotic objects are known as black holes and their most distinctive feature, together with the emergence of curvature singularities, is the notion of event horizon that comes with them. Naively, one can understand an event horizon as the region that causally separates the interior of the black hole from the rest of the universe, namely it is a region beyond which events cannot affect an external observer.

Now, let us try to be a bit more precise with the definitions of black hole and event horizon. First let us define some preliminary notions 30,85

Definition 1. Let $(\mathcal{M}, g)$ be a time-orientable spacetime, and let $U \subset \mathcal{M}$. Then,

1. Chronological future of $U$ :

$$
I^{+}(U):=\left\{p \in \mathcal{M} \mid \exists \gamma_{t} \text { future-directed timelike curve , } \gamma_{t=0} \in U, t \geq 0\right\}
$$

and similarly for the chronological past of $U$, i.e. $I^{-}(U)$;

2. Causal future of $U$ :

$$
J^{+}(U):=\left\{p \in \mathcal{M} \mid \exists \gamma_{t} \text { future-directed causal curve , } \gamma_{t=0} \in U, t \geq 0\right\}
$$

and similarly for the causal past of $U$, i.e. $J^{-}(U)$;

Definition 2 (Asymptotically simple spacetimes). Let $(\mathcal{M}, g)$ be a time-orientable spacetime with no $C^{1}$ closed timelike curves. Then, $(\mathcal{M}, g)$ is asymptotically simple if $\exists(\overline{\mathcal{M}}, \bar{g})$ spacetime with boundary $\mathscr{I}$ whose interior is conformal to $(\mathcal{M}, g)$ with $\bar{g}=\Omega^{2}(x) g, \Omega>0$ on $\mathcal{M}$, that satisfy:

(i) $\left.\Omega\right|_{\mathscr{I}}=0$ and $\left.\mathrm{d} \Omega\right|_{\mathscr{I}} \neq 0$; 
(ii) Every maximally extended null geodesic in the interior of $M$ intersects $\mathscr{I}$ in exactly two points.

Then following $|30|$, one has that $\mathscr{I}$ consists of two null hypersurfaces $\mathscr{I}^{+}$and $\mathscr{I}^{-}$, respectively known as future null infinity and past null infinity, i.e. $\mathscr{I}=\mathscr{I}^{+} \cup \mathscr{I}^{-}$.

We are then ready to provide a formal definition for both black holes and event horizons, i.e.

Definition 3 (Black hole). Let $(\mathcal{M}, g)$ be an asymptotically flat spacetime, then a black hole region is defined as

$$
\mathcal{B H}:=\mathcal{M} \backslash\left\{\mathcal{M} \cap J^{-}\left(\mathscr{I}^{+}\right)\right\} .
$$

Definition 4 (Event horizon). Let $(\mathcal{M}, g)$ be an asymptotically flat black hole spacetime, then the future event horizon is defined as

$$
\mathscr{H}^{+}:=\mathcal{M} \cap \dot{J}^{-}\left(\mathscr{I}^{+}\right),
$$

where $\dot{J}^{-}\left(\mathscr{I}^{+}\right)$denotes the boundary of $J^{-}\left(\mathscr{I}^{+}\right)$in $\mathcal{M}$.

Remark. Similar definitions can be given for the white hole region and the past event horizon.

Now, from these definitions one can clearly infer that, in order to properly identify the notion of event horizon we need to know the whole future evolution of the system. Another important ingredient, that plays a key role in the singularity theorems [86, 87], is the notion of marginally outer trapped surface (MOTS).

First of all, let $(\mathcal{N}, g)$ be an asymptotically flat spacetime and let $\ell$ and $n$ be, respectively, the tangent vector to the outgoing null geodesics and the tangent vector to the ingoing null geodesics. Furthermore, let $\Sigma \subset \mathcal{M}$ be a two-dimensional space-like surface formed by spatial foliations of the null hypersurface generated by $\ell$ and $n$. Then, denoting the induced metric on $\Sigma$ by $\left(\left.g\right|_{\Sigma}\right)_{\mu \nu} \equiv q_{\mu \nu}=g_{\mu \nu}+\ell_{\mu} n_{\nu}+n_{\mu} \ell_{\nu}$ (see e.g. 82, 83.) then one can define the expansion scalars associated with outgoing and ingoing geodesics as

$$
\Theta_{\ell}=q^{\mu \nu} \nabla_{\mu} \ell_{\nu}, \quad \Theta_{n}=q^{\mu \nu} \nabla_{\mu} n_{\nu}
$$

Definition 5 (MOTS). $\Sigma$ is a marginally outer trapped surface if

$$
\Theta_{\ell}=0, \quad \Theta_{n}<0, \quad \text { on } \Sigma .
$$

Remark. It is worth remarking that,

$$
\Theta_{\ell}=\frac{1}{\sqrt{q}} \mathfrak{L}_{\ell} \sqrt{q}, \quad \Theta_{n}=\frac{1}{\sqrt{q}} \mathfrak{L}_{n} \sqrt{q},
$$

with $\mathfrak{L}$ denoting the Lie derivative and $q:=\operatorname{det}\left(q_{\mu \nu}\right)$. Then, recalling that $q$ is a scalar, one also finds that $\Theta_{\ell}=(1 / \sqrt{q}) \ell^{\mu} \partial_{\mu} \sqrt{q}$ and $\Theta_{n}=(1 / \sqrt{q}) n^{\mu} \partial_{\mu} \sqrt{q}$. 


\subsubsection{Spherical symmetry and quasi-local mass}

Let us now focus our attention on the spacial case of spherically symmetric spacetimes, as they provide a framework in which the notion of quasi-local energy and MOTS are well understood and appear to be strictly intertwined.

First, let us recall that a spacetime $(\mathcal{M}, g)$ is said to be spherically symmetric if and only if it admits an $S O(3)$ group of isometries. If we denote by $S^{2}(R)$ a sphere of radius $R>0$ in the Euclidean space, this is a 2-manifold that, in spherical coordinates, has an induced metric $g_{S^{2}}$ given by the line element

$$
\mathrm{d} \sigma^{2}=R^{2} \mathrm{~d} \Omega^{2}=R^{2}\left(\mathrm{~d} \theta^{2}+\sin ^{2} \theta \mathrm{d} \phi^{2}\right) .
$$

From the last expression, one can immediately see that $g_{S^{2}}$ admits three Killing vector fields, namely

$$
\begin{aligned}
& \xi_{1}=-\sin \phi \partial_{\theta}-\cot \theta \cos \phi \partial_{\phi}, \\
& \xi_{2}=\cos \phi \partial_{\theta}-\cot \theta \sin \phi \partial_{\phi}, \\
& \xi_{3}=\partial_{\phi}
\end{aligned}
$$

such that

$$
\left[\xi_{a}, \xi_{b}\right]=-\varepsilon_{a b c} \xi_{c},
$$

i.e. closing the $\mathfrak{s o}(3)$ algebra. Now, the condition for $(\mathcal{M}, g)$ to be a spherically symmetric spacetime is $S O(3) \triangleright g=g$, or equivalently

$$
\mathfrak{L}_{\varpi} g=0, \quad \varpi=\sum_{i} a_{i} \xi_{i}, \quad a_{i} \in \mathbb{R}, \quad \forall i \in\{1,2,3\},
$$

with $\mathfrak{L}_{\varpi}$ denoting the Lie derivative with respect to the vector field $\varpi$. From the last condition one can easily infer that the most general line element for a spherically symmetric spacetime, in spherical coordinates, is given by

$$
\mathrm{d} s^{2}=g_{A, B}\left(x^{1}, x^{2}\right) \mathrm{d} x^{A} \mathrm{~d} x^{B}+r^{2}\left(x^{1}, x^{2}\right) \mathrm{d} \Omega^{2},
$$

where $A, B=1,2, g_{A, B}\left(x^{1}, x^{2}\right)$ is equipped with a Lorentzian signature and $r^{2}\left(x^{1}, x^{2}\right)$ denotes the area radius.

Given all these premises, we can now define the Misner-Sharp [88] or quasi-local mass 89 91] as

$$
m:=\frac{r}{2 G_{\mathrm{N}}}\left(1-g^{A B} \nabla_{A} r \nabla_{B} r\right) .
$$

It is now worth recalling a few properties of this object:

1. If the spacetime is static, then $m$ is constant on each round sphere. 
2. If we choose $x^{1}=t$ and $x^{2}=r$ as local coordinates on $g_{A B}$, then we find

$$
g^{r r}=1-\frac{2 G_{\mathrm{N}} m}{r},
$$

and thus, for a generic spherically symmetric space we have

$$
\mathrm{d} s^{2}=-e^{-2 \Phi}\left(1-\frac{2 G_{\mathrm{N}} m}{r}\right) \mathrm{d} t^{2}+\left(1-\frac{2 G_{\mathrm{N}} m}{r}\right)^{-1} \mathrm{~d} r^{2}+r^{2} \mathrm{~d} \Omega^{2},
$$

for some function $\Phi=\Phi(t, r)$.

3. If we plug (3.14) into in the Einstein equations, we get

$$
G_{0}^{0}=8 \pi G_{\mathrm{N}} T_{0}^{0} \equiv-8 \pi G_{\mathrm{N}} \rho,
$$

which implies

$$
\frac{\mathrm{d} m}{\mathrm{~d} r}=4 \pi r^{2} \rho,
$$

with $\rho=\rho(t, r)$ denoting the energy density of the matter source. Hence, we have that

$$
m(t, r)=4 \pi \int_{0}^{r} \rho(t, \bar{r}) \bar{r}^{2} \mathrm{~d} \bar{r} .
$$

This last result tells us that the Misner-Sharp mass can be interpreted as the total energy contained in a sphere of radius $r$. Besides, since the integral is performed using a flat measure, i.e. $4 \pi r^{2} \mathrm{~d} r$, no information about the background geometry is required in order to compute this quantity.

4. If the spacetime is static, then

$$
\lim _{r \rightarrow \infty} m(r)=M,
$$

where $M$ is the $A D M$ mass.

Another important feature of the quasi-local mass, in spherical symmetry, is that it allows to locate MOTS in a very simple way. Indeed, one can prove that

Theorem 5. Let $(\mathcal{M}, g)$ be a spherically symmetric asymptotically flat spacetime, then all MOTS are solutions of $2 G_{\mathrm{N}} m(t, r)=r$ (or, equivalently, are roots of $\left.g^{r r}=0\right)$.

However, if the spherical symmetry is spoiled no general notion of quasi-local mass with such (physical) properties is known, even though a few proposals have appeared in the literature, see e.g. [92]. 


\subsubsection{HQM for spherical systems}

Let us start with a discussion of the HQM formalism, in both its global and local formulations, for static and spherically symmetric black holes. This section, in particular, is based on $\mid 78$ and hence we invite the interested reader to refer to this article for the specific details of the analysis summarized here.

The aim of this technique is to provide a fully fledged quantum mechanical description of MOTS and, to do that, the key idea is to investigate what happens to these surfaces if one assumes the matter component that generates the spacetime to be a quantum mechanical object. In other words, we are interested in the effects on MOTS due to the quantum mechanical nature of the energy-momentum tensor. Now, if the source behave like a purely quantum mechanical system, then quantities like the quasi-local mass and the ADM mass should be promoted to quantum observables. Hence, it is reasonable to expect that the geometry of the spacetime, understood in terms of the notion of gravitational (or Schwarzschild) radius, will inherit some degree of quantum fuzziness from the nature of the source. In other words, we expect to be able to describe, within a certain degree of approximation, the gravitational radius as quantum observable as well.

Since in gravity both the gravitational field and its source are notions that appear strongly entangled to one another, then the Hilbert space for the whole system can be defined, in a very general manner, as

$$
\mathcal{H}:=\mathcal{H}_{S} \otimes \mathcal{H}_{G}
$$

with $\mathcal{H}_{S}$ and $\mathcal{H}_{G}$ denoting the Hilbert space for the source and for the geometry, respectively.

Now, in this framework the simplest way to reproduce the strict intertwining between matter and gravity, at the quantum level, is obtained by considering an entangled state

$$
\mathcal{H} \ni|\Psi\rangle=\sum_{\alpha, \beta} C\left(E_{\alpha}, R_{\mathrm{H} \beta}\right)\left|E_{\alpha}\right\rangle\left|R_{\mathrm{H} \beta}\right\rangle
$$

where the quantum states of the "matter" correspond to the spectral decomposition of a certain hamiltonian operator $\widehat{H}$ (precisely, $\widehat{H} \otimes \mathbb{I}_{G}$ ), whereas the quantum states of the geometry are labelled in terms of the spectral decomposition of a gravitational radius operator $\widehat{R}_{\mathrm{H}}$ (namely, $\mathbb{I}_{S} \otimes \widehat{R}_{\mathrm{H}}$ ). Or, in simpler terms, the ADM mass and the Schwarzschild radius have been lifted to operators on $\mathcal{H}$. However, not all states of $\mathcal{H}$ are physically relevant. Indeed, within this formalism, the physical states of $\mathcal{H}$ are the one that can be written as in (3.19) and such that

$$
\left(\widehat{H}-\frac{1}{2 G_{\mathrm{N}}} \widehat{R}_{\mathrm{H}}\right)|\Psi\rangle_{\text {phys }}=0,
$$

i.e. that the one that allow to recover the relation between the ADM mass and the gravitational radius. If we then plug the definition 3.19 in the constraint 3.20 , one finds

$$
C\left(E_{\alpha}, R_{\mathrm{H} \beta}\right)=C\left(E_{\alpha}, 2 G_{\mathrm{N}} E_{\alpha}\right) \delta_{\alpha \beta}
$$


Then, in this picture, if one wishes to recover all the information about the source, one just need to trace out the gravitational degrees of freedom, i.e.

$$
|\Psi\rangle_{\mathrm{S}}=\sum_{\gamma} C_{S}\left(E_{\gamma}\right)\left|E_{\gamma}\right\rangle, \quad C_{S}\left(E_{\gamma}\right) \equiv C\left(E_{\gamma}, 2 G_{\mathrm{N}} E_{\gamma}\right)
$$

hence recovering the spectral decomposition of the source, as expected. Moreover, an this is rather more instructive, tracing out the source one finds

$$
|\Psi\rangle_{\mathrm{H}}=\sum_{\gamma} C_{S}\left(R_{\mathrm{H} \gamma} / 2 G_{\mathrm{N}}\right)\left|R_{\mathrm{H} \gamma}\right\rangle
$$

The latter is known in the literature as the horizon wave-function (HWF) and represents the key ingredient of the HQM formalism.

The power of this approach resides in the possibility of providing a probabilistic notion of black hole. Indeed,

Definition 6. (Probabilistic definition of black hole) Let $\widehat{H}$ and $\widehat{R}_{\mathrm{H}}$ be operators with continuous spectra on $\mathcal{H}$ (for sake of simplicity, these arguments can easily be extended also to the case of discrete spectra). Then:

(i) HWF in the gravitational radius representation

$$
\Psi_{\mathrm{H}}\left(R_{\mathrm{H}}\right):=\left\langle R_{\mathrm{H}} \mid \Psi_{\mathrm{H}}\right\rangle=\mathcal{N}_{\mathrm{H}} C_{S}\left(R_{\mathrm{H} \gamma} / 2 G_{\mathrm{N}}\right),
$$

with $\mathcal{N}_{\mathrm{H}}$ denoting a normalization constant.

(ii) Horizon probability density function

$$
\mathcal{P}_{\mathrm{H}}\left(r_{\mathrm{H}}\right):=4 \pi r_{\mathrm{H}}^{2}\left|\Psi_{\mathrm{H}}\left(r_{\mathrm{H}}\right)\right|^{2} .
$$

(iii) Black hole probability density function

$$
\mathcal{P}_{<}\left(r<r_{\mathrm{H}}\right):=\mathcal{P}_{\mathrm{S}}\left(r<R_{\mathrm{H}}\right) \mathcal{P}_{\mathrm{H}}\left(R_{\mathrm{H}}\right),
$$

where

$$
\mathcal{P}_{\mathrm{S}}\left(r<r_{\mathrm{H}}\right)=4 \pi \int_{0}^{R_{\mathrm{H}}} \bar{r}^{2}\left|\Psi_{\mathrm{S}}(\bar{r})\right|^{2} \mathrm{~d} \bar{r} .
$$

(iv) Black hole probability

$$
P_{\mathrm{BH}}:=\int_{0}^{\infty} \mathcal{P}_{<}\left(r<r_{\mathrm{H}}\right) \mathrm{d} r_{\mathrm{H}} .
$$

Remark. It is worth remarking that 3.24 represents the probability density function that one would detect a gravitational radius of size $r_{\mathrm{H}}$ associated with the "particle" in the quantum state $|\Psi\rangle_{\mathrm{S}}$. Besides, 3.25 denotes the probability density for a quantum "particle" to be a black hole, i.e. that the quantum size of the source falls within $r_{\mathrm{H}}$. 
So far we have discussed what happens if we consider spacetimes for which we have control on the global structure of the geometry and of which we know the whole evolution of the system. However, as stressed in Section 3.1.2, a much more interesting concept is represented by the notion of MOTS, which appear naturally equipped with local features.

In order to study MOTS with the HQM we need a local notion of gravitational radius, i.e. related to localised energy eigenmodes (that would naturally be related to discrete energy quantum numbers). Luckily, for spherical system we have a well defined notion of "local energy" for a self-gravitating system, which is ultimately given by the quasi-local mass. Thus, one can readily modify the HQM formalism for spherical systems in order to provide a tool for investigating MOTS at the quantum level. Indeed, if we focus on what happens within a sphere of radius $r$, the role the ADM mass is now played by the quasi-local mass $m(r)$, as discussed in Section 3.1.2. Hence, if we make the replacement $m(r) \mapsto \widehat{H}(r)$, with $\widehat{H}(r)$ denoting a sort of local Hamiltonian operator for the source, and proceed as above one finds

$$
\widehat{H}(r)=\sum_{\alpha} P_{\alpha}(r) E_{\alpha}\left|E_{\alpha}\right\rangle\left\langle\left. E_{\alpha}\left|, \quad P_{\alpha}(r):=4 \pi \int_{0}^{r} \bar{r}^{2}\right| \Psi_{E_{\alpha}}(\bar{r})\right|^{2} \mathrm{~d} \bar{r} .\right.
$$

Remark. It is important to stress that

$$
\lim _{r \rightarrow \infty} P_{\alpha}(r)=1,
$$

only if $\Psi_{E_{\alpha}} \in L_{2}\left(\mathbb{R}^{+}\right)$, namely for localised energy eigenmodes. Of course, this specific restriction turns out to be not necessary in the global case since the norm of these modes never enters explicitly in the analysis involving the hamiltonian operator.

Now, if we keep following the same guidelines that we presented for the global case, we find ourselves with enough ingredients to provide a local (quantum-mechanical) characterization of MOTS, specifically one finds

$$
\left\langle r_{\mathrm{H}}\left|\widehat{r}_{\mathrm{H}}(r)\right| r_{\mathrm{H}}\right\rangle=r, \quad r_{\mathrm{H} \alpha}(r):=P_{\alpha}(r) R_{\mathrm{H} \alpha},
$$

which is the local quantum version the condition $g^{r r}=0$ in terms of the quasi-local mass.

Example 1 (Single Gaussian wave-packet sourcing Schwarzschild). Let us consider a massive quantum particle, at rest in the origin of the reference frame, described by a spherically symmetric Gaussian wave-function, i.e.

$$
\Psi_{\mathrm{S}}(r)=\frac{1}{\left(\ell^{2} \pi\right)^{3 / 4}} \exp \left(-\frac{r^{2}}{2 \ell^{2}}\right)
$$

where $r=|\boldsymbol{x}|$ and assuming $\ell=\lambda_{m}=\ell_{\mathrm{P}} m_{\mathrm{P}} / m$ the Compton(-de Broglie) wavelength of the particle. The Fourier transform turns a function in the coordinate space $f(\boldsymbol{x})$ into one in the momentum space $\widetilde{f}(\boldsymbol{p})=\mathscr{F}[f(\boldsymbol{x}) ; \boldsymbol{p}]$, hence we can easily infer the form of the wave-function for the source 3.30 in the momentum space, that reads

$$
\widetilde{\Psi}_{\mathrm{S}}(\boldsymbol{p})=\frac{1}{\left(m^{2} \pi\right)^{3 / 4}} \exp \left(-\frac{|\boldsymbol{p}|^{2}}{2 m^{2}}\right) \text {. }
$$


If we assume, for simplicity, the validity of the relativistic flat mass-shell relation, i.e. $E^{2}=|\boldsymbol{p}|^{2}+m^{2}$, then (3.31) entails the spectral decomposition of the source since, roughly speaking, one has that

$$
\Psi_{\mathrm{S}}(\boldsymbol{x}) \sim \int \mathrm{d}^{3} p e^{i \boldsymbol{x} \cdot \boldsymbol{p}} \widetilde{\Psi}_{\mathrm{S}}(\boldsymbol{p}) \sim \int \mathrm{d} E \varphi_{E}(\boldsymbol{x}) C_{\mathrm{S}}(E),\left.\quad C_{\mathrm{S}}(E) \sim \widetilde{\Psi}_{\mathrm{S}}(\boldsymbol{p})\right|_{|\boldsymbol{p}|^{2}=E^{2}-m^{2}},
$$

with $\varphi_{E}(\boldsymbol{x})$ denoting the eigenfunctions of an appropriate hamiltonian operator.

At this point, the constraint (3.20) tell us that $R_{\mathrm{H}}=2 \ell_{\mathrm{P}} m / m_{\mathrm{P}}$ and $r_{\mathrm{H}}=2 \ell_{\mathrm{P}} E / m_{\mathrm{P}}$. Hence, plugging the flat mass-shell relation into (3.31), implementing the last remarks coming from the constraint and fixing the normalization on finds that

$$
\Psi_{\mathrm{H}}\left(r_{\mathrm{H}}\right)=\frac{1}{4 \ell_{\mathrm{P}}^{3}} \sqrt{\frac{m_{\mathrm{P}}^{3} \ell_{\mathrm{P}}^{3}}{\pi m^{3} \Gamma(3 / 2,1)}} \Theta\left(r_{\mathrm{H}}-R_{\mathrm{H}}\right) \exp \left(-\frac{m_{\mathrm{P}}^{2}}{8 m^{2} \ell_{\mathrm{P}}^{2}} r_{\mathrm{H}}^{2}\right),
$$

with the Heaviside distribution appearing in order to implement the condition $E \geq m$.

Now, if we compute all the quantities introduced in Definition 6, then we con plot the black hole probability $P_{\mathrm{BH}}$ as a function of the mass of the particle, see Figure 3.1. From Figure 3.1 one can immediately see that such a quantum particle is likely to be a black hole provided that its mass $m \gtrsim m_{\mathrm{P}}$ or, in other terms, $\lambda_{m} \lesssim \ell_{\mathrm{P}}$. This results appears to be perfectly consistent with what one would expect from a naive argument based sorely on semiclassical gravity and the uncertainty principle. Thus this example provides an important consistency check for the HQM formalism.

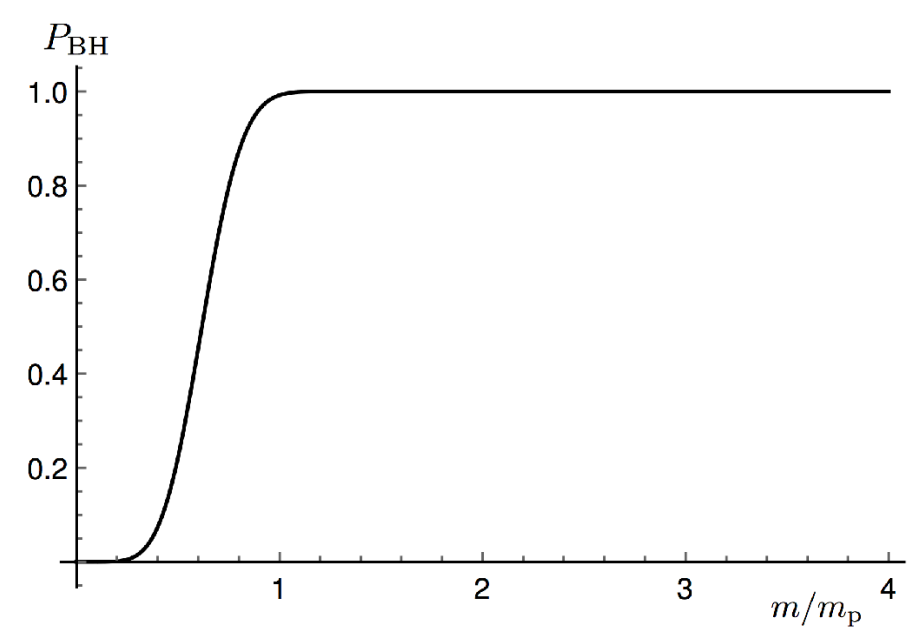

Figure 3.1: Plot of $P_{\mathrm{BH}}$ as a function of the mass of the particle.

Remark. This formalism has been applied to several different spherically symmetric scenarios, therefore we invite the interested reader to refer to [72 77 ) for many other insightful examples. 


\subsubsection{HQM for rotating systems}

A generalization of the HQM to Kerr black holes was first introduced in [79]. Let us recall some of the key features of rotating black holes belonging to the Kerr family. First of all, the Kerr spacetime 93, 94 is a solution of the vacuum Einstein equations given by the metric

$$
\begin{aligned}
\mathrm{d} s^{2} & =-\left(1-\frac{r_{s} r}{\Sigma}\right) \mathrm{d} t^{2}+\frac{\Sigma}{\Delta} \mathrm{d} r^{2}+\Sigma \mathrm{d} \theta^{2} \\
& +\left(r^{2}+a^{2}+\frac{r_{s} r a^{2}}{\Sigma} \sin ^{2} \theta\right) \sin ^{2} \theta \mathrm{d} \phi^{2}-\frac{2 r_{s} r a \sin ^{2} \theta}{\Sigma} \mathrm{d} t \mathrm{~d} \phi
\end{aligned}
$$

with $M$ denoting the ADM mass, $r_{s}=2 G_{\mathrm{N}} M$ the Schwarzschild "parameter", $a=J / M$ the angular momentum density corresponding to the total angular momentum $J$ of the system, $\Sigma=r^{2}+a^{2} \cos ^{2} \theta$ and $\Delta=r^{2}-r_{s} r+a^{2}$.

Studying the structure of MOTS in this spacetime [95, one can easily see that its event horizon is determined by the condition $\Delta(r)=0$, whose solutions are given by

$$
R_{\mathrm{H}}^{( \pm)}=G_{\mathrm{N}}\left(M \pm \sqrt{M^{2}-\frac{J^{2}}{M^{2}}}\right) .
$$

Furthermore, it is also worth remarking that in the early ' 70 , Carter and Robinson independently provided proofs of the uniqueness theorem [96, 97] for Kerr black holes, that states

Theorem 6 (No-hair for rotating black holes). The only possible stationary, axisymmetric and asymptotically flat black hole solutions of the vacuum Einstein equations are the Kerr solutions (3.33) subject to the constraint $M^{2}-a^{2}>0$.

Therefore, black holes of this kind are completely characterized by two real parameters: $(M, J)$.

Following the argument presented in Section 3.1.3, the natural way to proceed would involve promoting $(M, J)$ to operators on a suitable Hilbert space $\mathcal{H}$. However, one should be extremely careful while going down this road since the notion of angular momentum in quantum mechanics differs a lot from the classical one. Hence, the correct way of tackling this problem is to assume the existence of a complete set of commuting operators related to the quantum source $\left\{\widehat{H}, \widehat{J}^{2}, \widehat{J}_{z}\right\}$, respectively the Hamiltonian, the total angular momentum squared and the angular momentum along the axis of rotation. Then, the quantization procedure can be implemented by means of the replacements

$$
\begin{gathered}
M \mapsto \widehat{H}=\sum_{a, j, m} E_{a j}|\operatorname{aj} m\rangle\langle\operatorname{aj} m|, \\
J^{2} \mapsto \widehat{J}^{2}=m_{\mathrm{p}}^{4} \sum_{a, j, m} j(j+1)|a j m\rangle\left\langle a j m\left|\equiv \sum_{a, j, m} \lambda_{j}\right| a j m\right\rangle\langle a j m|,
\end{gathered}
$$




$$
\widehat{J}_{z}=m_{\mathrm{p}}^{2} \sum_{a, j, m} m|a j m\rangle\left\langle a j m\left|\equiv \sum_{a, j, m} \xi_{m}\right| a j m\right\rangle\langle a j m| .
$$

where $j \in \mathbb{N}_{0} / 2, m \in \mathbb{Z} / 2$, with $|m| \leq j$, and $a \in \mathcal{J}$, with $\mathcal{J}$ a discrete set of labels that can be either finite of infinite.

Remark. Clearly here the states $|a j m\rangle$ belong to $\mathcal{H}_{\mathrm{S}}$ and we are omitting the tensor product with the identity operator of $\mathcal{H}_{\mathrm{G}}$ for operators acting only on $\mathcal{H}_{\mathrm{S}}$ for sake of simplicity.

Following the procedure outlined in Section 3.1.3 for spherically symmetric spacetimes, the quantum state for the whole system can here be defined as a triple entangled state

$$
|\Psi\rangle=\sum_{a, j, m} \sum_{\alpha, \beta} C\left(E_{a j}, \lambda_{j}, \xi_{m}, R_{\mathrm{H}_{\alpha}^{(+)}}^{(+)} R_{\mathrm{H}_{\beta}}^{(-)}\right)|a j m\rangle|\alpha\rangle_{+}|\beta\rangle_{-}
$$

where $|\alpha\rangle_{ \pm}$are the eigenstates of $\widehat{R}_{\mathrm{H}}^{( \pm)}$.

Now, one can generalize the constraint equation 3.20 to the axisymmetric case by requiring the physically relevant states of $\mathcal{H}$ to satisfy

$$
\left(\widehat{R}_{\mathrm{H}}^{(+)}-\widehat{\mathcal{O}}^{+}\right)|\Psi\rangle_{\text {phys }}=0, \quad\left(\widehat{R}_{\mathrm{H}}^{(-)}-\widehat{\mathcal{O}}^{-}\right)|\Psi\rangle_{\text {phys }}=0,
$$

with

$$
\widehat{\mathcal{O}}^{ \pm}:=\widehat{H} \pm\left(\widehat{H}^{2}-\widehat{J}^{2} \widehat{H}^{-2}\right)^{1 / 2}
$$

provided that $\widehat{\mathcal{O}}^{ \pm}$is positively semi-definite. One can then follow Definition 6 and introduce an HWF for each horizon and, consequently, compute the black hole probability. We shall see an example of application for this technique in Section 4.4.3.

Before concluding this section, it is important to remark that, differently from the spherically symmetric case, we cannot easily perform a local analysis of quantum mechanical effects for the horizons since we lack of a general and well defined notion of quasi-local mass for general spaces.

\subsection{Selfcompletion via Classicalization}

The main feature of effective field theory (EFT) is that it represents a set of general techniques whose main goal is to single out the most suitable (quantum) degrees of freedom that would serve as an adequate reference to describe a system at a given energy scale. From a QFT perspective, we understand as "suitable degrees of freedom" the one that allow us to describe the system by means of a perturbative picture, i.e. to work in a weak coupling regime. Of course, any EFT is naturally equipped with a certain domain of applicability (usually expressed as an energy scale), beyond which the theory breaks down and we therefore need to "complete" this description by means of a more powerful and general theory. 
As a working example, let us consider Fermi's interaction [98,99]. This theory is given by a Dirac Lagrangian together with a four-fermion interaction term, i.e. $\mathcal{L}_{\text {int }} \sim G_{F}(\bar{\Psi} \Psi)^{2}$. Now, from a naive dimensional analysis one can immediately see that $[\Psi]=\operatorname{mass}^{1 / 2}$. length $^{-1}$ (from the kinetic term) which implies $\left[G_{F}\right]=\left[G_{\mathrm{N}}\right]=$ length $\cdot$ mass $^{-1}$ or, in Planck units (i.e. $c=\hbar=G_{\mathrm{N}}=k_{\mathrm{B}}=1$ ), $\left[G_{F}\right]=\operatorname{mass}^{-2}$ (since in this set of units energy $=$ mass $=$ momentum $=$ length $^{-1}$ ). Hence, we can write $G_{F}=\hbar / \Lambda^{2}$ where $\Lambda$ represent an intrinsic energy scales of the theory.

In QFT it is well known that theories involving interactions whose coupling constants have negative mass dimensions (in Planck units) are non-renormalizable. This is the case of Fermi theory, indeed once the center of mass energy of a certain process approaches $\Lambda$, then all the orders of the perturbative expansion of an arbitrary amplitude $\mathcal{M}$ become relevant leading to a violation of unitarity (i.e. the cross-section diverges). In other words, since all the terms of the perturbative expansion have to be taken into account, the degrees of freedom that we have adopted, so far, for describing the system are no more weaklyinteracting, but they have rather entered a strong coupling regime. Hence, these degrees of freedom do not provide, in principle, a suitable description of the physics for energies $E \gtrsim \Lambda$, and we therefore need an UV-completion of the theory.

In the (standard) Wilsonian approach [100,101], the UV-completion of a theory is achieved by integrating in some new degrees of freedom in a way that, ultimately, allows to recover a weak coupling regime. An example of application of this approach is the formulation of the electroweak theory, which represents the UV-completion of the Fermi theory of weak interactions. Indeed, the sick behaviour of Fermi's interaction in the UV is cured by introducing three vector bosons $\left(W^{ \pm}\right.$and $\left.Z^{0}\right)$ acting as mediators of the weak interaction. Hence, one can understand Fermi theory as the low energy limit of the more general electroweak theory. However, in this work we wish to consider a non-Wilsonian approach, based on the ideas of selfcompletion and classicalization, see e.g. 102 104. Roughly speaking, the new scheme can be summarized as follows:

Let us assume that there exists an energy scale $\Lambda$ beyond which the relevant degrees of freedom of our effective theory become strongly coupled. Then, instead of introducing some new degrees of freedom to restore the weak coupling regime, one can assume that the theory completes itself by producing some high-multiplicity states of the same particles that were already in the theory. Each of these constituents would then be extremely soft and weakly interacting with one another. Moreover, since the occupation number of the newly produced soft quanta is extremely high, the resulting state would approximately behave classically.

In this picture, the role of the potential "new degrees of freedom", that one would expect in the Wilsonian approach, is then taken up by the collective effect of the many weakly-interacting soft quanta produced in the process, which also act as fundamental constituents of the resulting quasi-classical final state. This is why we can say that such a theory selfcompletes in the UV by classicalization.

It was argued in 102, 105, 106 that Einstein's gravity might represent a perfect playground for the classicalization scheme. Indeed, the idea that many-particle states might represent the right path toward a quantum mechanical description of self-gravitating sys- 
tems is something that emerges naturally, for example, from the HQM formalism. As a matter of fact, if we recall the discussion in Example 1, one can readily compute the uncertainty in the horizon radius as,

$$
\Delta R_{\mathrm{H}}^{2}:=\left\langle\Psi_{H}\left|\widehat{R}_{\mathrm{H}}^{2}\right| \Psi_{H}\right\rangle-\left\langle\Psi_{H}\left|\widehat{R}_{\mathrm{H}}\right| \Psi_{H}\right\rangle^{2}
$$

thus, for the single particle case one finds [77,

$$
\Delta R_{\mathrm{H}} \sim \lambda_{m}^{-1} \sim m \sim R_{\mathrm{H}}
$$

This tell us that if $m \gg m_{\mathrm{P}}$, then the magnitude of the quantum fluctuations of the horizon are of the size of the horizon itself. This is clearly not acceptable since, in this regime, black holes are expected to behave (semi-)classically. Therefore, this very simple argument suggests that the single particle description of black holes, at the quantum level, breaks down when the energy scale exceeds the Planck energy.

Let us then provide a qualitative description of black hole formation from high energy scattering processes based on the classicalization scheme (see e.g. 107,108]). Hence, for the sake of argument, let us consider a collision of two elementary particles with center of mass energy $\sqrt{s} \gg m_{\mathrm{P}}$. We shall also assume the validity of Thorne's Hoop Conjecture (see e.g. [109, 110] for the classical formulation and $[72$ for its quantum mechanical extension), which states that a back hole forms in a collision if the two colliding objects fall within their "black disk", i.e. if the system reaches a point where it occupies a region of size $r$ such that $r \lesssim R_{\mathrm{H}}(s) \equiv \ell_{\mathrm{P}} \sqrt{s} / m_{\mathrm{P}}$. Then, if all the aforementioned assumptions are fulfilled, one can argue that the main features of classicalization for gravity are enclosed in a $2 \rightarrow N$ scattering process, where the two high energy quanta scatter producing $N \gg 1$ soft virtual gravitons with typical quantum size $\lambda_{\mathrm{G}} \simeq R_{\mathrm{H}}(s)$ (or energy $\epsilon_{\mathrm{G}}=\hbar / \lambda_{\mathrm{G}} \simeq \hbar / R_{\mathrm{H}}(s)$ ). Now, because of the conservation of energy, one finds that $\sqrt{s}=N \epsilon_{\mathrm{G}}$ which implies $N \simeq s / m_{\mathrm{P}}^{2} \gg 1$.

Denoting with $\alpha(\boldsymbol{p}):=G_{\mathrm{N}}|\boldsymbol{p}|^{2} / \hbar$ the dimensionless effective coupling for a momentum exchange $\boldsymbol{p}$ (for a precise discussion of the running of the gravitational coupling see e.g. [111-113|), one can immediately see that the effective coupling of the $N$ gravitons in the final state is given by

$$
\alpha_{\mathrm{G}}=\frac{G_{\mathrm{N}}\left|\boldsymbol{p}_{\mathrm{G}}\right|^{2}}{\hbar}=\frac{\hbar G_{\mathrm{N}}}{\lambda_{\mathrm{G}}^{2}} \simeq \frac{m_{\mathrm{P}}^{2}}{s} \simeq \frac{1}{N},
$$

where, in the second equality, we used the de Brogile relation $\lambda_{\mathrm{G}}=\hbar /\left|\boldsymbol{p}_{\mathrm{G}}\right|$. The latter tells us that while the system is globally in a strong coupling regime, as indicated by the collective (dimensionless) coupling $g=\alpha_{\mathrm{G}} N \simeq 1$, each constituent interacts very weakly with the other fundamental elements of ths system. In other words, the system of $N$ gravitons resulting from the scattering, in the lenguage of Bose-Einstein condensates, is at its quantum critical point $[114-116]$. This means that, despite the fact that the constituents of the final state are weakly interacting, non-perturbative effects due to the collective interaction among the gravitons become extremely relevant and lead to the global (approximately) classical behaviour of the system. 
It is worth remarking that the rate of the $2 \rightarrow N$ process has been computed, in the kinematic regime, in $[106$ and its behaviour is given by

$$
\Gamma_{2 \rightarrow N} \sim \alpha_{\mathrm{G}}^{N} N ! \sim e^{-N}, \quad N \gg 1 .
$$

Such an exponential suppression is indeed perfectly consistent with the expected entropy suppression factor that one would find for a transition from a two-particle state to a black hole micro-state, in the traditional semiclassical picture. Indeed, since the BekensteinHawking entropy scales as $S_{\mathrm{BH}} \sim R_{\mathrm{H}}^{2} \sim N$, thus $\mathcal{M}(2 \rightarrow N) \sim e^{-S_{\mathrm{BH}}} \sim e^{-N}$. Besides, one can also observe that $S_{\mathrm{BH}} \sim N \sim 1 / \alpha_{\mathrm{G}}$, namely the entropy scales like the inverse of the coupling, this implies that $\mathcal{M}(2 \rightarrow N) \sim e^{-1 / \alpha_{\mathrm{G}}}$.

\subsubsection{Black Hole's Quantum N-Portrait}

Starting from the previous discussion, we can draw an interesting (non-geometric) quantum picture for black holes 107,108.

For the sake of argument, let us try to form a graviton wave-packet made of as many gravitons as possible, let us say $N$, with a typical wavelength $\lambda_{\mathrm{G}} \gg \ell_{\mathrm{P}}$. Clearly, since

$$
\alpha_{\mathrm{G}}=\frac{\hbar G_{\mathrm{N}}}{\lambda_{\mathrm{G}}^{2}}=\frac{\ell_{\mathrm{P}}^{2}}{\lambda_{\mathrm{G}}^{2}} \ll 1,
$$

this bunch of gravitons will interact very weakly with one another. However, if you put together several gravitons, each of them will start perceiving a collective potential produced by the other $N-1$ constituents of the system.

The collective binding potential felt by each graviton, at first order of approximation, can be estimated to be given by $U \simeq-\alpha_{\mathrm{G}} N \hbar / \lambda_{\mathrm{G}}$, for $r \simeq \lambda_{\mathrm{G}}$. Besides, each graviton will have kinetic energy given by $K \simeq\left|\boldsymbol{p}_{\mathrm{G}}\right| \simeq \hbar / \lambda_{\mathrm{G}}$. Hence, if we want to form a bound state out of these $N$ gravitons one just needs to require the energy balance $K+U \simeq 0$ (marginally bound condition) for each graviton, which implies

$$
\alpha_{\mathrm{G}} N \simeq 1
$$

which is the same scaling that we found in the classicalization picture. Thus, given a black hole of ADM mass $M$, we can understand it, in this "corpuscular picture", as a self-sustained bound state (or Bose-Einstein condensate) of $N$ gravitons with typical wavelength $\lambda_{\mathrm{G}} \simeq R_{\mathrm{H}}$ and mutual interaction strength $\alpha_{\mathrm{G}}=1 / N$. Now, since $\alpha_{\mathrm{G}}=\ell_{\mathrm{P}}^{2} / \lambda_{\mathrm{G}}^{2}$, then $\lambda_{\mathrm{G}} \simeq \sqrt{N} \ell_{\mathrm{P}}$ which implies $M \simeq \sqrt{N} m_{\mathrm{P}}$. If we then put everything together, we get the so called scaling laws of corpuscular black holes, i.e.

$$
M=\sqrt{N} m_{\mathrm{P}}, \quad \lambda_{\mathrm{G}} \simeq \sqrt{N} \ell_{\mathrm{P}}, \quad \alpha_{\mathrm{G}} \simeq \frac{1}{N} .
$$

Another important thing to stress is that, as discussed above, the quantum criticality condition $\alpha_{\mathrm{G}} N \simeq 1$ tells us that what we are dealing with is a self-sustained bound state on 
the verge of a quantum phase transition. Furthermore, because of the quantum criticality, the system is characterized by collective Bogoliubov modes, lightly gapped i.e. $\Delta \epsilon \sim 1 / N$, which are responsible for carrying the entropy of the black hole [114].

Now, since this bound state is formed at the verge of decay, so it cannot really hold on to its constituents. Thus the system is continuously loosing gravitons through a quantum depletion process, as one would expect for an interacting homogeneous Bose-Einstein condensate [117]. Besides, this leakage behaves like a corpuscular quantum precursor of Hawking's radiation. Indeed, let us consider the $2 \rightarrow 2$ scattering of two constituent gravitons in which one of them gains enough energy to leave the ground state, namely leaves the condensate in this case. The rate for such a process is simply given by $\Gamma \sim \alpha_{\mathrm{G}}^{2} N(N-1) \epsilon_{\mathrm{G}} / \hbar$ (see [107, 108|), that yields

$$
\Gamma \simeq \frac{1}{\sqrt{N} \ell_{\mathrm{P}}}+\mathcal{O}\left(\frac{1}{\ell_{\mathrm{P}} N^{3 / 2}}\right)
$$

form which we get the depletion law

$$
\frac{\mathrm{d} N}{\mathrm{~d} t} \simeq-\frac{1}{\sqrt{N} \ell_{\mathrm{P}}}+\mathcal{O}\left(\frac{1}{\ell_{\mathrm{P}} N^{3 / 2}}\right),
$$

that, through the scaling laws (3.45), can be rewritten in terms of the ADM mass $M$ as

$$
\frac{\mathrm{d} M}{\mathrm{~d} t} \simeq-\frac{m_{\mathrm{P}}^{3}}{\ell_{\mathrm{P}} M^{2}}+\mathcal{O}\left(\frac{m_{\mathrm{P}}^{5}}{\ell_{\mathrm{P}} M^{3}}\right) \simeq-\frac{T^{2}}{\hbar}+\mathcal{O}\left(\frac{T^{4}}{\hbar m_{\mathrm{P}}^{2}}\right),
$$

where $T:=\hbar / G_{\mathrm{N}} M=\hbar / \sqrt{N} \ell_{\mathrm{P}}$. The result in (3.47) then matches Hawking's argument in the semiclassical limit, i.e. $N \rightarrow \infty, \ell_{\mathrm{P}} \rightarrow 0$, keeping $\sqrt{N} \ell_{\mathrm{P}}$ and $\hbar$ finite.

The major difference between Hawking's theory and the corpuscular picture is that, in the latter case, Hawking's radiation has nothing to do with thermodynamics, since we are dealing with a cold Bose-Einstein condensate and the temperature of the system is, actually, zero. In other words, corpuscular black holes evaporate though a cold quantum depleation process. Nonetheless, the fact that we recover a thermal spectrum in the semiclassical limit comes as a direct consequence of both combinatorics and the fact that the gravitons that deplete are soft 107.118. 


\section{Chapter 4}

\section{Corpuscular Black Holes}

In this Chapter we shall now try to refine the qualitative arguments introduced in Section 3.2 and 3.2 .1 by means of some effective toy models where the gravitons are replaced with massless scalar fields. Specifically, first we shall provide a more accurate description of black holes as self-sustained bound states, as well as of their relation with the emergence of non-trivial causal structures of the spacetime. To do that, we will employ the HQM formalism, since it allows to link the quantum features of the geometry of the spacetime to the behaviour of the quantum source, namely the self-sourcing of the graviton field in this case. Then, following the same general argument, we will discuss in detail the emergence of the Hawking effect in view of the quantum criticality of the system. After this general analysis, we will discuss what are the expected effect of this corpuscular model when dealing with the gravitational collapse of a matter source to form a black hole. Extending these argument, we will then study an effective quantum description of the static gravitational potential, for spherically symmetric systems, up to the first order in the post-Newtonian expansion. Further, we will provide a detailed study of the emerging geometrical structure of a rotating corpuscular black hole by means of the HQM formalism discussed in Section 3.1.4. Finally, we will conclude this Chapter by presenting some results concerning the physics of corpuscular black holes in lower dimensional spaces.

\subsection{Black holes as self-sustained quantum states}

The first precise characterization of the link between the notion of self-sustained bound states of soft gravitons and critical self-gravitating systems can be traced back to the pivotal works [119 121] by Casadio et al, that we will try to summarize here.

As suggested above, the simplest way to proceed in order to provide a more in-depth description of the quantum portrait for black holes, discussed in Section 3.2.1, is to use massless scalar fields as toy gravitons. So, let us consider a massless scalar field $\Phi(x)$ coupled to a (scalar) current $J(x)$ on the Minkowski space. The equation of motion for $\Phi(x)$ will then read

$$
\square \Phi(x)=J(x), \quad \square:=\eta^{\mu \nu} \partial_{\mu} \partial_{\nu},
$$


where $[\Phi]=$ length $^{-1}$.

Since, for the sake of simplicity, we will try to recover the classical and semiclassical features of the Schwarzschild solution, we shall consider a system which is both static and spherically symmetric. These two conditions should then reflect on the form of the source $J(x)$, namely we shall require that $\partial_{t} J(x)=0$ and $J(x) \equiv J(t, \boldsymbol{x})=J(t,|\boldsymbol{x}|)$.

Denoting with $k^{\mu}=\left(k^{0}, \boldsymbol{k}\right)$ the rescaled momentum and recalling that the radial Fourier transform of function $f(\boldsymbol{x})=f(r)$, with $r \equiv|\boldsymbol{x}|$, on $\mathbb{R}^{3}$ reads

$$
\widetilde{f}(\boldsymbol{k})=\int \mathrm{d}^{3} x e^{-i \boldsymbol{k} \cdot \boldsymbol{x}} f(\boldsymbol{x})=4 \pi \int_{0}^{\infty} j_{0}(\xi r) f(r) r^{2} \mathrm{~d} r \equiv \widetilde{f}(\xi),
$$

where $\xi \equiv|\boldsymbol{k}|, j_{0}(\xi r)=\sin (\xi r) / \xi r$ is the spherical Bessel function of the first kind of order $\nu=0$ and $[\boldsymbol{k}]=[\xi]=$ length $^{-1}$ (that is why we call it a rescaled momentum, since $[k]=[p / \hbar])$. Thus, the four dimensional Fourier transform reduces to

$$
\widetilde{f}(k)=\int \mathrm{d}^{4} x e^{-i k \cdot x} f(x)=4 \pi \int \mathrm{d} t e^{i k^{0} t} \int_{0}^{\infty} j_{0}(\xi r) f(t, r) r^{2} \mathrm{~d} r \equiv \int \mathrm{d} t e^{i k^{0} t} \widetilde{f}(t, \xi) .
$$

Remark. It is also worth stressing that $[\widetilde{f}(\boldsymbol{k})]=$ length $^{3} \cdot[f(\boldsymbol{x})]$ and $[\widetilde{f}(k)]=$ length $^{4} \cdot[f(x)]$.

All that said, the staticity of source then implies $k^{0} \widetilde{J}(k)=0$, which in turn is solved by $\widetilde{J}(k)=\delta\left(k^{0}\right) f(\boldsymbol{k})$ with $f^{*}(\boldsymbol{k})=f(-\boldsymbol{k})$ (reality conditions). For sake of simplicity, we will make the identification $f(\boldsymbol{k}) \equiv \widetilde{J}(\boldsymbol{k})$ that, for the time being, only represents the three dimensional Fourier transform of a certain function $J(\boldsymbol{x})$ on $\mathbb{R}^{3}$. The requirement of spherical symmetry then implies that $\widetilde{J}(\boldsymbol{k})=\widetilde{J}(\xi)$, thus the momentum representation of a static spherically symmetric scalar source $J(x)$ is given by $\widetilde{J}(k)=\delta\left(k^{0}\right) \widetilde{J}(\xi)$.

It is now easy to see that, form this very simple classical field theoretic setting one can recover an asymptotic Newtonian behaviour. Indeed, considering a Gaussian source, i.e. $J(r) \propto \exp \left[-r^{2} /\left(2 \sigma^{2}\right)\right]$, and solving for $\Phi(x)$ one finds $\Phi_{\mathrm{C}}(x)=\Phi_{\mathrm{C}}(r) \propto-(1 / r) \operatorname{erf}(r / \sqrt{2} \sigma)$, with erf denoting the error function $[122]$. It is then easy to see that $\Phi(r) \sim-1 / r$ as $r \gg \sigma$ thus, with suitable dimensional factors, one can make the identification $V_{N} \simeq$ $4 \pi G_{\mathrm{N}} M \Phi_{\mathrm{C}}(r)$ for $r \gg \sigma$. This means that, already with this very simple classical scalar theory, we recover a behaviour akin to Newton's gravity for a static and spherical symmetric source. Thus, this setting allows us to roughly mimic gravity with a masseless scalar field theory.

At the quantum level, classical solutions are recovered through (quantum) coherent states. Specifically, if we consider the (rescaled) Hamiltonian density in the momentum space

$$
: \mathcal{H}:=\xi{a^{\prime}}^{\dagger} a^{\prime}{ }_{\boldsymbol{k}}+\mathcal{E}_{g}, \quad \mathcal{E}_{g}=-\frac{|\widetilde{J}(\xi)|^{2}}{2 \xi^{2}}
$$

with $\mathcal{E}_{g}$ denoting the energy density of the ground state and $a^{\prime}{ }_{\boldsymbol{k}}$ the creation-annihilation operators shifted with respect to the standard one according to $a^{\prime}{ }_{\boldsymbol{k}}=a_{\boldsymbol{k}}+\widetilde{J}(\xi) / \sqrt{2 \xi^{3}}$. 
The ground state $|g\rangle$ is then defined according to $a_{\boldsymbol{k}}^{\prime}|g\rangle=0$ which, in terms of the standard creation-annihilation operators, reads $a_{\boldsymbol{k}}|g\rangle=-\left(\widetilde{J}(\xi) / \sqrt{2 \xi^{3}}\right)|g\rangle \equiv \alpha_{g}(\boldsymbol{k})|g\rangle$. Thus $|g\rangle$ is an "eigenstate" of $a_{\boldsymbol{k}}$, as expected for a coherent state. For such a state one can immediately compute the number of constituent quanta, which is given by

$$
N \simeq \int \mathrm{d}^{3} k\left\langle g\left|a_{\boldsymbol{k}}^{\dagger} a_{\boldsymbol{k}}\right| g\right\rangle \simeq \int \mathrm{d}^{3} k\left|\alpha_{g}(\boldsymbol{k})\right|^{2}
$$

Besides, it is easy to see that

$$
\langle g|\widetilde{\Phi}(k)| g\rangle=\widetilde{\Phi}_{\mathrm{C}}(\xi),
$$

thus recovering the classical result.

If we now wish to describe a star-like object of ADM mass $M$ and energy density $\rho(r)$ with this simplified model, we just need make the identification $J(r)=\rho(r) / M$ for the scalar current. The Newtonian potential energy for this star is then given by $U_{M}=M V_{\mathrm{N}}$, where $V_{\mathrm{N}}$ is determined through its classical relation with $\Phi_{C}$ and, therefore, by means of the quantum coherent state $|g\rangle$. It is then worth remarking that, for a sufficiently large mass $M$, the constituents of $|g\rangle$ carry an average energy $\epsilon \sim U_{M} / N$ while their total number $N \sim M^{2}$.

All that said, the black hole quantum $N$-portrait inherently assumes that the contribution of matter becomes negligible once the black hole has formed, whereas the confinement of the system is sourced by the self-interaction amongst the constituents. The latter can roughly be accounted for by means of a modified kinetic operator in (4.1). Hence, we just need to recast the marginally bound condition in a field theoretic form. Specifically, if take $\rho(r)=-N U_{\epsilon} /\left(4 \pi R^{3} / 3\right)$, i.e. the (positive) average total potential energy over the volume of the 2 -sphere of radius $R$ where the source has support (confinement), then

$$
J(r) \simeq-\frac{N G_{\mathrm{N}} \epsilon}{R^{3}} \Phi_{\mathrm{C}}(r)
$$

If we now take Eq. 4.1 to the momentum space and we then replace $J(r)$ with the expression (4.5), we get

$$
\xi^{2} \simeq \frac{N G_{\mathrm{N}} \epsilon}{R^{3}}
$$

Thus, recalling that $M=N \epsilon$ (i.e. the total energy equals the sum of the energies of the constituents) and denoting by $R_{\mathrm{H}}=2 G_{\mathrm{N}} M$ the gravitational radius associated with the mass $M$, we can rewrite the last equation as

$$
\xi^{2} \simeq \frac{R_{\mathrm{H}}}{R^{3}} .
$$

This clearly tell us that a self-sustained quantum system should only contain quanta with rescaled momentum

$$
\xi \simeq \frac{1}{R} \sqrt{\frac{R_{\mathrm{H}}}{R}} .
$$


If we then take the limit $R \rightarrow R_{\mathrm{H}}^{+}$, recalling that the typical wavelength of the constituents is $\lambda=\hbar /|\boldsymbol{p}|=1 /|\boldsymbol{k}| \equiv 1 / \xi$ and that $\epsilon \simeq|\boldsymbol{p}|=\hbar \xi$, we get

$$
\lambda \simeq R_{\mathrm{H}} \simeq \sqrt{N} \ell_{\mathrm{P}}, \quad \text { or, equivalently, } \quad \epsilon \simeq \frac{m_{\mathrm{P}}}{\sqrt{N}}
$$

as expected in for a corpuscular black hole.

It is now important to study the causal structure generated by this system. To do that we will employ the techniques of the HQM discussed in Section 3.1.3, for spherical systems.

First, in order to implement the formalism, we shall characterize the quantum state of the source. In this case, the system is made of $N$ marginally bound scalar particles, labelled by the index $i=1, \ldots, N$. The marginally bound condition can be implemented in the single-particle spectrum by requiring each quanta to have a single-particle Hilbert space containing a ground state $|\epsilon\rangle$, of energy $\epsilon=m_{\mathrm{P}} / \sqrt{N}$, and a continuous component of (quasi-)gapless excitations $\left|\omega_{i}\right\rangle$, of energy $\omega_{i}>\epsilon$. These quantum hair are meant to represent the quantum criticality of the system, i.e. the fact that the system is on the verge of a phase transition and quantum fluctuations can push the constituents out of the ground state (quantum depletion). Specifically, the single-particle wave-function for each constituent of the system, i.e. $\forall i=1, \ldots, N$, takes the form

$$
\left|\Psi_{\mathrm{S}}^{(i)}\right\rangle=\frac{|\epsilon\rangle+\gamma\left|\psi_{\text {cont. }}^{(i)}\right\rangle}{\sqrt{1+\gamma^{2}}}
$$

with $\gamma \geq 0$ denoting the likelihood for a particle to belong in the continuous part of the spectrum rather than in the ground state, and

$$
\left|\psi_{\text {cont. }}^{(i)}\right\rangle=\int_{\epsilon}^{\infty} \mathrm{d} \omega_{i} \varpi_{i}\left(\omega_{i} \mid \epsilon\right)\left|\omega_{i}\right\rangle
$$

where $\varpi_{i}\left(\omega_{i} \mid \epsilon\right)$ denotes the energy distribution function for the states in the continuum.

Then the total Hamiltonian and the $N$-particle wave-function for the system will be given by

$$
\widehat{H}=\bigoplus_{i=1}^{N} \widehat{H}^{(i)}, \quad\left|\Psi_{\mathrm{S}}\right\rangle=\bigotimes_{i=1, \ldots, N}^{\mathcal{S}}\left|\Psi_{\mathrm{S}}^{(i)}\right\rangle
$$

where $\widehat{H}^{(i)}$ is a single-particle Hamiltonian, characterized as above, and $\otimes^{\mathcal{S}}$ is a symmetrized tensor product (since we are dealing with a bosonic $N$-particle state). Therefore, the total Hamiltonian has a spectrum given by a discrete part, namely

$$
|M\rangle=\bigotimes_{i=1, \ldots, N}^{\mathcal{S}}|\epsilon\rangle, \quad \widehat{H}|M\rangle=M|M\rangle \text { with } M=N \epsilon
$$

and a continuous component such that $\widehat{H}|E\rangle=E|E\rangle$, with $E>M$. 
Since in the corpuscular model we want most of our constituent gravitons to be in the ground state, at least for most of the life of a black hole of mass $M \gg m_{\mathrm{P}}$, it is reasonable to work in the limit $\gamma \ll 1$, as in [119]. A more general discussion can the be found in 120,121. The coefficients $C_{\mathrm{S}}(E)$, defined in Section 3.1.3, then read [119,

$$
C_{\mathrm{S}}(E<M)=0, \quad C_{\mathrm{S}}(E=M) \simeq 1, \quad C_{\mathrm{S}}(E>M) \simeq \gamma \varpi_{i}\left(\omega_{i} \mid \epsilon, M\right) .
$$

Finally, specifying $\varpi_{i}\left(\omega_{i} \mid \epsilon\right)$ one can explicitly compute the probability for this system to form a black hole. Specifically, it is easy to see, for a Gaussian distribution centered around $\epsilon$, or for a thermal spectrum at a temperature $T \simeq \epsilon \simeq T_{\mathrm{H}}$, that $P_{\mathrm{BH}} \simeq 1$, computed according to Definition 6. This confirms the emergence of a non-trivial causal structure for such critical self-sustained states. Moreover, analyzing the system, separately, with both a Gaussian distribution centered around $\epsilon$ and a thermal spectrum at a temperature $T \simeq \epsilon$ tell us a lot more then just some general features of its causal structure. Indeed, in both cases, one finds that

$$
\frac{\Delta E}{\langle\widehat{H}\rangle_{\mathrm{S}}} \sim \frac{\gamma}{N}, \quad \frac{\Delta R_{\mathrm{H}}}{\left\langle\widehat{R}_{\mathrm{H}}\right\rangle_{\mathrm{H}}} \sim \frac{1}{N}
$$

in the limit $N \gg 1$, where $\Delta E^{2}:=\left\langle\widehat{H}^{2}\right\rangle_{\mathrm{S}}-\langle\widehat{H}\rangle_{\mathrm{S}}^{2}$ and $\Delta R_{\mathrm{H}}^{2}:=\left\langle\widehat{R}_{\mathrm{H}}^{2}\right\rangle_{\mathrm{H}}-\left\langle\widehat{R}_{\mathrm{H}}\right\rangle_{\mathrm{H}}^{2}$. This means that, for a black hole of mass $M \gg m_{\mathrm{P}}$ (i.e. $N \gg 1$ ), the quantum corrections to the classical GR picture are subject to a $1 / N$ suppression, which can be traced back to the softness of the constituents as anticipated in Section 3.2.1. Besides, the fact that, in the large $N$ limit, we recover the same thermal behaviour of the Hawking radiation, even if we consider quantum hair equipped with a non-thermal distribution. This confirms that the Hawking effect, in this model, is an emerging phenomena that can be traced back to combinatorics and the softness of the constituents.

Finally, it is worth remarking that this system has been fully characterized, at the thermodynamic level, in [120]. In this paper the authors computed the (effective) corpuscular corrections to the Bekenstein-Hawking entropy, whose behaviour is given by $S-S_{\mathrm{BH}} \sim \log N$ in the large $N$ limit, as well as the specific heat of the system.

\subsection{Gravitational collapse}

In this Section we will try to provide a simplified corpuscular description of the gravitational collapse following the general argument presented in 123.

First, let us first introduce a very naive model for astrophysical objects, for which we assume that a compact stellar object can be described in terms of $N_{\mathrm{B}}$ identical constituents, to which we refer to as baryons for simplicity, each one with a rest mass $\mu$. In particular, we wish to investigate spherically symmetric configurations of radius $R$ of this sort, assuming that we can neglect any emission of radiation. This last assumption guarantees the conservation of the total energy of this system, which in turns is reflected in the conservation of the ADM mass $M$. 
It is worth remarking that the conservation of energy, in classical gravity, is inherently linked to the time reparametrization invariance which, at the Hamiltonian level, is associated to the Hamiltonian constraint of GR $43,63,124,125]$. Thus, for an asymptotically flat spacetime, the Hamiltonian constraint of GR reads [63.124],

$$
H=H_{\mathrm{B}}+H_{\mathrm{G}}=M
$$

where $H_{\mathrm{B}}$ and $H_{\mathrm{G}}$ are the (super-)Hamiltonians for matter and gravity, respectively, while $M$ is the ADM mass (coming from boundary terms).

Now, there are two interesting cases for a self-gravitating spherically symmetric system described as above:

- Stable star, i.e. $R=R_{\mathrm{S}}$;

- Black hole, i.e. $R=R_{\mathrm{H}}=2 G_{\mathrm{N}} M$.

Note that, for the first case, the Hamiltonian constraint simply leads to the TolmanOppenheimer-Volkoff equation 126, 127.

Let us now try to present a general argument based on an energy balance for these selfgravitating objects. Hence, let us start from an initial configuration where all the baryons in the system are extremely far apart. Thus, the energy of the initial configuration is given by

$$
H_{\text {in }}=M \simeq \mu N_{\mathrm{B}} .
$$

If we then shrink the system down to a sphere of radius $R$ we find that

$$
H(R)=M+K_{\mathrm{B}}(R)+U_{\mathrm{BG}}(R)+U_{\mathrm{BB}}(R),
$$

where $K_{\mathrm{B}}(R)$ is the kinetic energy of the baryons, $U_{\mathrm{BG}}$ denotes the interaction potential energy between baryons mediated by the gravitons $\left(U_{\mathrm{BG}}(R) \leq 0\right)$ and $U_{\mathrm{BB}}(R) \geq 0$ is an additional repulsive interaction among baryons. In particular, the last term is responsible for the necessary pressure that allows the system to sustain static configurations.

In a purely classical framework $H_{\text {in }}=H(R)$, which implies

$$
K_{\mathrm{B}}(R)+U_{\mathrm{BG}}(R)+U_{\mathrm{BB}}(R)=0,
$$

however, baryons and gravitons are intrinsically quantum objects. So, let us consider the case of a static system set-up of size R, i.e. $K_{\mathrm{B}}(R)=0$. Hence, a Newtonian estimate for the baryon-baryon interaction mediated by gravitons gives,

$$
U_{\mathrm{BG}}(R) \simeq \mu N_{\mathrm{B}} V_{\mathrm{N}}(R) \simeq-\frac{G_{\mathrm{N}} M^{2}}{R},
$$

with $V_{\mathrm{N}}(R)$ denoting the Newtonian potential.

In Section 4.1 we have argued that the Newtonian potential can be obtained as a result of a coherent state of soft virtual gravitons [119]. Besides, for a self-gravitating object of 
ADM mass $M$, it was also shown that the number of quanta in this state is approximately given by

$$
N_{\mathrm{G}} \simeq \frac{M^{2}}{m_{\mathrm{P}}^{2}} \simeq \frac{R_{\mathrm{H}}^{2}}{\ell_{\mathrm{P}}^{2}},
$$

regardless for the size $R$ of the system, namely $N_{\mathrm{G}}$ does not depend on $R$.

In this picture, the gravitational interaction between baryons can now be written in terms of gravitons as

$$
U_{\mathrm{BG}}=-N_{\mathrm{G}} \epsilon_{\mathrm{G}},
$$

where $\epsilon_{\mathrm{G}}$ is the typical energy (positive) of the constituents of the coherent state while the minus sign tell us that the collective interaction is attractive.

Comparing 4.19) and (4.21), one finds

$$
\epsilon_{\mathrm{G}}(R) \simeq \frac{\hbar}{R}
$$

namely, the typical energy of the constituents of the coherent state is completely determined by its typical size $R$. In other words, while the total number of constituents of the state does not depend on the size of the system, the typical energy of its building blocks does.

Now, since gravitons self-interact, we should include a graviton-graviton interaction term, $U_{\mathrm{GG}}(R)$, in the total energy 4.17 , hence

$$
H(R)=M+K_{\mathrm{B}}(R)+U_{\mathrm{BG}}(R)+U_{\mathrm{GG}}(R)+U_{\mathrm{BB}}(R) .
$$

Since each of these $N_{\mathrm{G}}$ gravitons will interact with the coherent state trough the collective potential $V_{\mathrm{N}}$, we can then give a Newtonian estimate of the total energy contribution due to the interaction of each constituent with the collective state, i.e.

$$
U_{\mathrm{GG}}(R) \simeq N_{\mathrm{G}}\left[-\epsilon_{\mathrm{G}}(R)\right] V_{\mathrm{N}}(R) \simeq G_{\mathrm{N}}^{2} \frac{M^{3}}{R^{2}}
$$

which has the form of a post-Newtonian correction.

Note that,

$$
\left|\frac{U_{\mathrm{GG}}(R)}{U_{\mathrm{BG}}(R)}\right| \sim \frac{R_{\mathrm{H}}}{R},
$$

which is typically very small for a large star, i.e. $R \gg R_{\mathrm{H}}$. This means that the contribution of the self-interaction becomes relevant for very compact objects, i.e. $R \sim R_{\mathrm{H}}$.

It is now interesting to see what happens if we shrink the size $R$ of the system down to its gravitational radius $R_{\mathrm{H}}$. What we find is that,

$$
U_{\mathrm{GG}}\left(R_{\mathrm{H}}\right) \simeq-U_{\mathrm{BG}}\left(R_{\mathrm{H}}\right) \simeq M
$$


that leads to

$$
\alpha_{\mathrm{G}} N_{\mathrm{G}} \sim 1
$$

which is exactly the expected quantum criticality condition (where, again, $\alpha_{\mathrm{G}}=\epsilon_{\mathrm{G}}^{2}\left(R_{\mathrm{H}}\right) / m_{\mathrm{P}}^{2}$ is the dimensionless effective graviton coupling). Besides, since the general condition (4.20) implies $R_{\mathrm{H}} \simeq \sqrt{N_{\mathrm{G}}} \ell_{\mathrm{P}}$, it is easy to see that

$$
\epsilon_{\mathrm{G}}\left(R_{\mathrm{H}}\right) \simeq \frac{m_{\mathrm{P}}}{\sqrt{N_{\mathrm{G}}}}
$$

thus recovering the scaling of the typical energy (effective mass) of the gravitons in the condensate.

For sake of completeness, it is worth remarking that we have employed some simple Newtonian arguments to infer the form of $U_{\mathrm{BG}}$ and $U_{\mathrm{GG}}$. However, one could potentially improve the vindication of the results presented above by taking profit of some more accurate potentials for self-gravitating system, such as the harmonic [128 or the PöschlTeller 129 potential.

\subsection{Corpuscular post-Newtonian theory}

We shall now try to refine the arguments presented in Section 4.2 by constructing an effective theory for the gravitational potential of a static spherical symmetric system up to the first post-Newtonian correction. We will than map this emerging theory into a scalar field theory, from which we can recover the classical Newtonian potential as a result of a quantum coherent state. Beside, we further analyse the form of the corrections to the coherent state required to properly accommodate the expected (classical) post-Newtonian correction.

It is worth remarking that this Section is mostly based on $130-132$ so, for the details on the computations, we invite the interested reader to refer to these articles.

\subsubsection{Classical effective scalar theory}

Let us start by considering the post-Newtonian expansion of the Schwarzschild metric at the level of the Einstein-Hilbert action (2.6) coupled to some matter Lagrangian density $\mathcal{L}_{\mathrm{M}}$, i.e.

$$
S=S_{\mathrm{EH}}+\int \mathrm{d}^{4} x \sqrt{-\operatorname{det} g} \mathcal{L}_{\mathrm{M}}
$$

In this framework, the post-Newtonian approximation is obtained by working in the weak field regime, i.e. $g_{\mu \nu}=\eta_{\mu \nu}+h_{\mu \nu}$ with $\left|h_{\mu \nu}\right| \ll 1$, together with the assumption that the matter component of the system moves with a non-relativistic characteristic velocity. For the sake of accuracy, it is important to stress that all these assumptions have to hold in the 
chosen reference frame for static observers. Under these assumptions, and in this reference frame, all the key information about the gravitational filed are carried by $h_{00}(\boldsymbol{x})$. Besides, the energy-momentum tensor for the non-relativistic matter component is given in terms of the energy density by $T_{\mu \nu} \simeq \rho(\boldsymbol{x}) u_{\mu} u_{\nu}$, with $u \equiv \partial_{t}$ (or, in other words, $u^{\mu}=\delta_{0}^{\mu}$ ). This is true since we are not interested in the matter dynamics, but rather only on how this static distribution of matter generates the gravitational field in the non-relativistic limit in which the matter pressure is negligible [133, 134]. Now, it is easy to see that this $T_{\mu \nu}$ follows from a very simple matter Lagrangian density, i.e. $\mathcal{L}_{\mathrm{M}} \simeq-\rho(\boldsymbol{x})$. Recalling that the equations of motion for $h_{\mu \nu}$, in the de Donder gauge, read

$$
-\square h_{\mu \nu}=16 \pi G_{\mathrm{N}}\left(T_{\mu \nu}-\frac{1}{2} \eta_{\mu \nu} T\right), \quad T:=\eta^{\mu \nu} T_{\mu \nu},
$$

then, within the framework discussed above, we find

$$
\Delta h_{00}(\boldsymbol{x})=-8 \pi G_{\mathrm{N}} \rho(\boldsymbol{x}),
$$

with $\Delta$ denoting the Laplace operator, that reduces to the Poisson equation for the Newtonian potential $V_{\mathrm{N}}(\boldsymbol{x})$,

$$
\Delta V_{\mathrm{N}}(\boldsymbol{x})=4 \pi G_{\mathrm{N}} \rho(\boldsymbol{x}),
$$

once we make the identification $h_{00}(\boldsymbol{x}) \equiv-2 V_{\mathrm{N}}(\boldsymbol{x})$.

Starting from this last result, it is now easy to formulate an effective scalar field theory for the gravitational potential. Indeed, considering a static spherically symmetric system we have that $V_{\mathrm{N}}(\boldsymbol{x}) \equiv V_{\mathrm{N}}(r)$ and $\rho(\boldsymbol{x}) \equiv \rho(r)$, with $r$ denoting the radial coordinate. Now, since the construction of effective theories from expansions of the Einstein-Hilbert action coupled to matter, beyond the linear order, presents several technical and conceptual difficulties, the formulation of the model presented here will be built strating from the (massless) Fierz-Pauli action (2.29), see [130] for further details. Hence, we obtain the (approximated) Lagrangian

$$
\begin{aligned}
L\left[V_{\mathrm{N}}\right] & \simeq \int \mathrm{d}^{3} x \mathcal{L}_{\mathrm{FP}+\mathrm{M}} \simeq 4 \pi \int_{0}^{\infty} r^{2} \mathrm{~d} r\left(\frac{1}{32 \pi G_{\mathrm{N}}} h_{00} \Delta h_{00}+\frac{h_{00}}{2} T_{00}\right) \\
& \simeq 4 \pi \int_{0}^{\infty} r^{2} \mathrm{~d} r\left(\frac{1}{8 \pi G_{\mathrm{N}}} V_{\mathrm{N}} \Delta V_{\mathrm{N}}-\rho V_{\mathrm{N}}\right) \\
& \simeq-4 \pi \int_{0}^{\infty} r^{2} \mathrm{~d} r\left[\frac{1}{8 \pi G_{\mathrm{N}}}\left(V_{\mathrm{N}}^{\prime}\right)^{2}+\rho V_{\mathrm{N}}\right],
\end{aligned}
$$

where the prime denotes the derivative with respect to $r$. It is now easy to see that the Poisson equation is obtained from 4.33 by varying the resulting action with respect to $V_{\mathrm{N}}(r)$.

In order to push this effective model beyond the Newtonian limit, we need to include the self-sourcing of the gravitational filed, namely adding non-linear terms to the Lagrangian 
(4.33). The first step in this direction is to compute the effective Hamiltonian from (4.33), i.e.

$$
\begin{aligned}
H\left[V_{\mathrm{N}}\right]=-L\left[V_{\mathrm{N}}\right] & \simeq 4 \pi \int_{0}^{\infty} r^{2} \mathrm{~d} r\left(-\frac{1}{8 \pi G_{\mathrm{N}}} V_{\mathrm{N}} \Delta V_{\mathrm{N}}+\rho V_{\mathrm{N}}\right) \\
& \simeq 4 \pi \int_{0}^{\infty} r^{2} \mathrm{~d} r\left[\frac{1}{8 \pi G_{\mathrm{N}}}\left(V_{\mathrm{N}}^{\prime}\right)^{2}+\rho V_{\mathrm{N}}\right]
\end{aligned}
$$

If we then evaluate the first line on-shell (namely, plugging in the Poisson equation) we get the Newtonian potential energy, i.e.

$$
U_{\mathrm{N}}(R) \simeq 2 \pi \int_{0}^{R} r^{2} \mathrm{~d} r \rho(r) V_{\mathrm{N}}(r),
$$

understood as the energy due to the gravitational interaction of the matter distribution with the gravitational field within a 2-sphere of radius $R$. On the other hand, using again the Poisson equation, it is easy to spot that

$$
U_{\mathrm{N}}(R) \simeq-\frac{1}{2 G_{\mathrm{N}}} \int_{0}^{R} r^{2} \mathrm{~d} r\left[V_{\mathrm{N}}^{\prime}(r)\right]^{2},
$$

hence, if we now follow the arguments presented in Section 4.2, we have that the source of the self-gravitating features of the system is linked to the Newtonian energy density, namely

$$
J\left[V_{\mathrm{N}} ; r\right]=\frac{1}{4 \pi r^{2}} \frac{d}{d r} U_{\mathrm{N}}(r)=-\frac{\left[V^{\prime}{ }_{\mathrm{N}}(r)\right]^{2}}{8 \pi G_{\mathrm{N}}} .
$$

It is now worth remarking that a contribution of this form does indeed appear in the next-to-leading order of the expansion of the Einstein-Hilbert theory coupled to matter, see [130 for a detailed proof of this statement.

A more refined analysis of the post-Newtonian expansion of 4.29 , up to the next-toleading order contribution, then allows us to properly include the self-sourcing term $J[V ; r]$ into our effective scalar theory, giving rise to the improved Lagrangian

$$
\bar{L}[V]=4 \pi \int_{0}^{\infty} r^{2} \mathrm{~d} r\left[\frac{1}{8 \pi G_{\mathrm{N}}} V \Delta V-q_{\mathrm{B}} \rho V+2 q_{\Phi}\left(q_{\mathrm{B}} V \rho-2 J[V ; r] V\right)\right],
$$

or, in simpler terms,

$$
\bar{L}[V]=-4 \pi \int_{0}^{\infty} r^{2} \mathrm{~d} r\left[\frac{1}{8 \pi G_{\mathrm{N}}}\left(1-4 q_{\Phi} V\right)\left(V^{\prime}\right)^{2}+q_{\mathrm{B}} V \rho\left(1-2 q_{\Phi} V\right)\right],
$$

where $q_{\mathrm{B}}$ and $q_{\Phi}$ are just some dimensionless parameters to keep track of the coupling of $V$ with matter and the self-coupling, respectively. The corresponding equation of motion is then given by,

$$
\left(1-4 q_{\Phi} V\right) \Delta V=4 \pi q_{\mathrm{B}} G_{\mathrm{N}} \rho\left(1-4 q_{\Phi} V\right)+2 q_{\Phi}\left(V^{\prime}\right)^{2} .
$$


Then, the on-shell improved Hamiltonian reads,

$$
\bar{H}[V]=2 \pi \int_{0}^{\infty} \mathrm{d} r r^{2}[\underbrace{q_{\mathrm{B}} \rho V\left(1-4 q_{\Phi} V\right)}_{\text {Baryon-Graviton }}-\underbrace{\frac{3 q_{\Phi}}{2 \pi G_{\mathrm{N}}} V\left(V^{\prime 2}\right)}_{\text {Graviton-Graviton }}]+\mathcal{O}\left(q_{\Phi}^{2}\right),
$$

that can be split into two contributions, namely the one containing the matter density, i.e.

$$
U_{\mathrm{BG}}=2 \pi q_{\mathrm{B}} \int_{0}^{\infty} r^{2} \mathrm{~d} r \rho\left[V_{(0)}+q_{\Phi}\left(V_{(1)}-4 V_{(0)}^{2}\right)\right]+\mathcal{O}\left(q_{\Phi}^{2}\right),
$$

and a second one due to the self-sourcing,

$$
U_{\mathrm{GG}}=-3 q_{\Phi} \frac{\ell_{\mathrm{P}}}{m_{\mathrm{P}}} \int_{0}^{\infty} r^{2} \mathrm{~d} r V_{(0)}\left(V_{(0)}^{\prime}\right)^{2}+\mathcal{O}\left(q_{\Phi}^{2}\right) .
$$

Remark. Note that if we stet $q_{\mathrm{B}}=1$ and $q_{\Phi}=0$ we recover Eq. 4.36, as expected.

Now, since the problem concerning the general solution of 4.40$)$ is rather involved, we shall try to see what happens if we treat $q_{\Phi} V$ and $q_{\Phi}\left(V^{\prime}\right)^{2}$ as perturbations to the Poisson equation. Thus, let $V_{(0)}(r)$ be such that

$$
\Delta V_{(0)}=4 \pi G_{\mathrm{N}} q_{\mathrm{B}} \rho,
$$

then, let us expand $V(r)$ up to first order in the coupling $q_{\Phi}$, i.e.

$$
V(r)=V_{(0)}(r)+q_{\Phi} V_{(1)}(r)+\mathcal{O}\left(q_{\Phi}^{2}\right) .
$$

Plugging this ansatz into 4.40 we find

$$
\Delta V_{(1)}=2\left(V_{(0)}^{\prime}\right)^{2}
$$

whose solution gives the correction of order $\mathcal{O}\left(q_{\Phi}\right)$ to the Newtonian estimates.

Example 2 (Uniform matter distribution). Let us try to make some more explicit statements by considering a specific matter distribution. Specifically, let us consider a matter source which is uniformly distributed within a sphere of radius $R$, i.e.

$$
\rho(r)=\frac{3 M_{0}}{4 \pi R^{3}} \Theta(R-r)
$$

with $M_{0}$ the mass of the source and $\Theta$ the Heaviside theta distribution.

By employing the whole analysis presented above one finds a total gravitational energy

$$
U(R)=U_{\mathrm{BG}}+U_{\mathrm{GG}} \simeq-q_{\mathrm{B}}^{2} \frac{3 G_{\mathrm{N}} M^{2}}{5 R}+q_{\mathrm{B}}^{3} q_{\Phi} \frac{249 G_{\mathrm{N}}^{2} M^{3}}{175 R^{2}}+\mathcal{O}\left(q_{\Phi}^{2}\right),
$$

with $M$ being the ADM, given by

$$
M=M_{0}\left(1+q_{\Phi} q_{\mathrm{B}} \frac{12 \ell_{\mathrm{P}} M_{0}}{5 m_{\mathrm{P}} R}\right)+\mathcal{O}\left(q_{\Phi}^{2}\right)
$$


see [130 for all the details.

From Eq. 4.46 we immediately see that the total gravitational energy is consistent with the estimates in Section 4.2. Besides, the post-Newtonian energy (namely, the term of order $q_{\Phi}$ in this theory) is positive and becomes comparable to the Newtonian contribution (i.e. term of order $q_{\Phi}^{0}$ in this model) if

$$
R \simeq 1.2 R_{\mathrm{H}}
$$

where we set $q_{\mathrm{B}}=q_{\Phi}=1$. Thus, we recover the maximal packing condition for $R \sim R_{\mathrm{H}}$. Nonetheless, it is worth noting that this value of $R$ falls outside the regime of validity of our approximation [130,132]. However, some better working example can be found in [130].

\subsubsection{Quantum uplifting of the model}

Now, let us try to see how we can reproduce these classical results in a quantum theory. To do that we will quantize canonically an appropriate rescaled potential and then we will try to infer the form of the quantum state that appropriately yields expectation values close to the classical picture discussed above.

Since we wish to recast our effective theory 4.38 into a canonically normalized scalar field theory, we have to make the replacements

$$
\Phi=\frac{1}{\sqrt{G_{\mathrm{N}}}} V, \quad J_{\mathrm{B}}=4 \pi \sqrt{G_{\mathrm{N}}} \rho,
$$

which lead to a rescaled improved Lagrangian,

$$
\begin{aligned}
L[\Phi]=4 \pi \int_{0}^{\infty} r^{2} \mathrm{~d} r\left[\frac{1}{2} \Phi \square \Phi\right. & -q_{\mathrm{B}} J_{\mathrm{B}} \Phi\left(1-2 q_{\Phi} \sqrt{G_{\mathrm{N}}} \Phi\right) \\
& \left.+4 \pi q_{\Phi} \sqrt{G_{\mathrm{N}}}\left(\partial_{\mu} \Phi\right)^{2} \Phi\right],
\end{aligned}
$$

that we denote by $L$ instead of $\bar{L}$ for sake of simplicity and where we, again, assumed $\Phi=\Phi(t, r)$.

Let us first focus on the "Newtonian case", namely setting $q_{\Phi}=0$. With this assumption the (classical) equation of motion reduces to the Poisson equation

$$
\Delta \Phi_{\mathrm{C}}(r)=q_{\mathrm{B}} J_{\mathrm{B}}(r) .
$$

We can then bring this equation to the momentum space, denoting $k \equiv|\boldsymbol{k}|$, as

$$
\widetilde{\Phi}_{\mathrm{C}}(k)=-q_{\mathrm{B}} \frac{\widetilde{J_{\mathrm{B}}}(k)}{k^{2}},
$$

and we can consequently define the coherent state $|g\rangle$ as

$$
a_{\boldsymbol{k}}|g\rangle=\exp \left(i \gamma_{\boldsymbol{k}}(t)\right) g_{\boldsymbol{k}}|g\rangle
$$


with

$$
g_{\boldsymbol{k}}=\sqrt{\frac{k}{2 \hbar}} \widetilde{\Phi}_{\mathrm{C}}(k)=-q_{\mathrm{B}} \frac{\widetilde{J_{\mathrm{B}}}(k)}{\sqrt{2 \hbar k^{3}}},
$$

in strict analogy with the procedure laid down in Section 4.1. Now, it is easy to see that

$$
\langle g|\Phi(t, r)| g\rangle=\Phi_{\mathrm{C}}(r),
$$

assuming $\langle g \mid g\rangle=1$ and $\gamma_{\boldsymbol{k}}(t)=-k t$, see [130]. Besides, from the normalization it is also possible to read-off the number of quanta in the coherent state, namely

$$
N_{\mathrm{G}}=\int_{0}^{\infty} \frac{k^{2} \mathrm{~d} k}{2 \pi^{2}}\left\langle g\left|a_{\boldsymbol{k}}^{\dagger} a_{\boldsymbol{k}}\right| g\right\rangle=\int_{0}^{\infty} \frac{k^{2} \mathrm{~d} k}{2 \pi^{2}}\left|g_{\boldsymbol{k}}\right|^{2} .
$$

If we then consider a source of mass $M$ of finite spatial extension $R$, one finds [130]

$$
N_{\mathrm{G}} \sim \frac{M^{2}}{m_{\mathrm{P}}^{2}} \log \left(\frac{R_{\infty}}{R}\right),
$$

with $R_{\infty}=k_{0}^{-1} \gg R$ denoting an infrared cut-off connected with the size of the Universe $R_{\infty}$, within which the gravitational field is static. It is also important to observe that the dependence of $N_{\mathrm{G}}$ is clearly weaker on $R$ than on the mass $M$. This allows us to recover the scaling $N_{\mathrm{G}} \sim M^{2} / m_{\mathrm{P}}^{2}$ when the size of the Universe is arbitrarily large.

So far we have found that

$$
\sqrt{G_{\mathrm{N}}}\langle g|\Phi(t, r)| g\rangle=V_{\mathrm{N}}(r)=V_{(0)}(r),
$$

it is now interesting to see what happens to $V_{(1)}(r)$ at the quantum level. Clearly, we can rewrite Eq. (4.44) as,

$$
\Delta V_{(1)}=2 G_{\mathrm{N}}\left\langle g\left|\left(\Phi^{\prime}\right)^{2}\right| g\right\rangle .
$$

Besides, it is easy to see that,

$$
\begin{aligned}
2 G_{\mathrm{N}}\left\langle g\left|\left(\Phi^{\prime}\right)^{2}\right| g\right\rangle & =8 \ell_{\mathrm{P}}^{2}\left[\int_{0}^{\infty} \frac{k^{5 / 2} \mathrm{~d} k}{2 \sqrt{2} \pi^{2}} j_{1}(k r) g_{k}\right]^{2}+2 \ell_{\mathrm{P}}^{2} \int_{0}^{\infty} \frac{k^{3} \mathrm{~d} k}{4 \pi^{2}}\left[j_{1}(k r)\right]^{2} \\
& \equiv J_{g}+J_{0},
\end{aligned}
$$

with $j_{1}$ denoting the spherical of the first kind of order one.

It is then worth noting that the term $J_{0}$ is just a (diverging) vacuum contribution that can be removed by normal ordering, besides form Eq. 4.53) one can immediately infer that

$$
J_{g}=2 G_{\mathrm{N}}\left\langle g\left|\left(\Phi^{\prime}\right)^{2}\right| g\right\rangle=2\left(V_{(0)}^{\prime}\right)^{2},
$$


for any current sourcing the scalar field. This clearly underlines the fact that $|g\rangle$ represent a solid starting point for our perturbative quantum analysis. Indeed, it is now interesting to see what kind of modification of the coherent state $|g\rangle$, obtained from the Newtonian potential, are required in order to reproduce the first post-Newtonian correction, i.e.

$$
\sqrt{G_{\mathrm{N}}}\left\langle g^{\prime}|\Phi(t, r)| g^{\prime}\right\rangle=V_{(0)}(r)+q_{\Phi} V_{(1)}(r)
$$

for some $\left|g^{\prime}\right\rangle \simeq \mathcal{N}\left(|g\rangle+q_{\Phi}|\delta g\rangle\right)$ with $a_{\boldsymbol{k}}\left|g^{\prime}\right\rangle \simeq g_{\boldsymbol{k}}|g\rangle+q_{\Phi} \delta g_{\boldsymbol{k}}|\delta g\rangle$ and $|\mathcal{N}|^{2} \simeq 1-$ $2 q_{\Phi} \operatorname{Re}\langle\delta g \mid g\rangle$. Plugging in the approximate expressions for $\left|g^{\prime}\right\rangle$ we find,

$$
\left\langle g^{\prime}|\Phi(t, r)| g^{\prime}\right\rangle \simeq\langle g|\Phi| g\rangle+2 q_{\Phi} \operatorname{Re}\langle\delta g|\Phi| g\rangle-2 q_{\Phi}\langle g|\Phi| g\rangle \operatorname{Re}\langle\delta g \mid g\rangle
$$

that, because of 4.57) and 4.61, reduces to

$$
\operatorname{Re}\langle\delta g|\Phi| g\rangle-\langle g|\Phi| g\rangle \operatorname{Re}\langle\delta g \mid g\rangle \simeq \frac{V_{(1)}(r)}{2 G_{\mathrm{N}}}
$$

By applying the Laplace operator to both sides, we get

$$
\Delta \operatorname{Re}\langle\delta g|\Phi| g\rangle-\operatorname{Re}\langle\delta g \mid g\rangle \Delta\langle g|\Phi| g\rangle=\sqrt{G_{\mathrm{N}}}\left\langle g\left|\left(\Phi^{\prime}\right)^{2}\right| g\right\rangle
$$

where we have also taken profit of 4.60 .

We shall now try to solve (4.64) in order to identify the relation between the eigenvalues $\delta g_{\boldsymbol{k}}$ and the one corresponding to the Newtonian coherent state, i.e. $g_{\boldsymbol{k}}$. In order to simplify our discussion, let us assume that almost the whole set of toy gravitons belongs to one mode of wavelength $\bar{\lambda} \simeq R$, as in the previous Sections, then after some manipulations one finds

$$
\delta g_{\overline{\boldsymbol{k}}} \sim-\ell_{\mathrm{P}} \bar{k}^{5 / 2} g_{\overline{\boldsymbol{k}}}^{2}
$$

with $\bar{k} \simeq \delta \bar{k} \simeq 1 / R$

Example 3 (Gaussian source, see [130]). If one considers the case of a Gaussian source,

$$
J_{\mathrm{B}}(r)=\frac{4 \sqrt{G_{\mathrm{N}}} M_{0}}{\pi^{1 / 2} \sigma^{3}} \exp \left(-\frac{r^{2}}{\sigma^{2}}\right),
$$

setting $\bar{k} \simeq \sigma^{-1}$ one finds,

$$
\delta g_{\bar{k}} \sim \frac{R_{\mathrm{H}}}{\sigma} g_{\bar{k}}
$$

which means that $\delta g_{\bar{k}} \ll g_{\bar{k}}$ for a source that is much more extended than its gravitational radius $\left(R \simeq \sigma \gg R_{\mathrm{H}}\right.$ ), which is indeed consistent with the classical results we were trying to reproduce at the quantum mechanical level. 


\subsection{Rotating and non-singular black holes}

In this Section, after reviewing the harmonic model of corpuscular black holes by Casadio and Orlandi [128], we will employ the HQM formalism to analyse some features of the Kerr and Hayward spacetimes within the quantum $N$-portrait.

It is important to stress that the HQM plays a fundamental role in this study since, starting from an effective description of the state produced by the constituent gravitons, it allows to infer the likelihood for a certain effective geometry to properly describe the causal structure of the system.

\subsubsection{Quantum harmonic black holes}

As discussed in Section 4.1 and 4.2, in the corpuscular model black holes are understood as some sort of Bose-Einstein condensates of marginally bound soft virtual gravitons. The confinement of these particles is realized through their Newtonian potential energy $U_{\mathrm{N}}$ and the marginally bound condition is then given by

$$
\epsilon_{\mathrm{G}}+U_{\mathrm{N}} \simeq 0 \text {. }
$$

A first rough approximation for the binding potential is given by a square well potential

$$
U_{\text {s.w. }}(r) \simeq U_{\mathrm{N}}\left(\lambda_{\mathrm{G}}\right) \simeq-\frac{N \alpha_{\mathrm{G}} \hbar}{\lambda_{\mathrm{G}}} \Theta\left(\lambda_{\mathrm{G}}-r\right)
$$

with $\lambda_{\mathrm{G}}=\hbar / \epsilon_{\mathrm{G}} \simeq R_{\mathrm{H}}$ and $\alpha_{\mathrm{G}}=\ell_{\mathrm{P}}^{2} / \lambda_{\mathrm{G}}^{2}=\epsilon_{\mathrm{G}}^{2} / m_{\mathrm{P}}^{2}$. If we then plug this approximated potential into 4.68 we recover the scaling laws 3.45 .

A more accurate approximation for the binding potential energy can be expressed in a form analogous to the harmonic potential, i.e.

$$
U_{\mathrm{h}}(r)=\bar{U}(r) \Theta(d-r), \quad \bar{U}(r):=\frac{1}{2} \mu \omega^{2}\left(r^{2}-d^{2}\right),
$$

see Figure 4.1 .

Now, making the identification $\mu=\epsilon_{\mathrm{G}}$, the parameters $d$ and $\omega$ need to be chosen in a way that guarantees that the highest energy state available to the gravitons is a marginally bound one. Neglecting the finite size of this harmonic well, the corresponding time-independent Schrödinger equation reads

$$
-\frac{\hbar^{2}}{2 \epsilon_{\mathrm{G}}} \Delta \psi+\bar{U}(r) \psi=\mathcal{E} \psi
$$

with $\Delta$ denoting the Laplace operator in spherical coordinates. Solving the latter eigenvalue problem one finds,

$$
\psi_{n j m}\left(r, \theta, \phi ; \lambda_{\mathrm{G}}\right)=\mathcal{N} r^{l} \exp \left(-\frac{r^{2}}{2 \lambda_{\mathrm{G}}^{2}}\right){ }_{1} F_{1}\left(-n, l+3 / 2, r^{2} / \lambda_{\mathrm{G}}^{2}\right) Y_{l m}(\theta, \phi),
$$




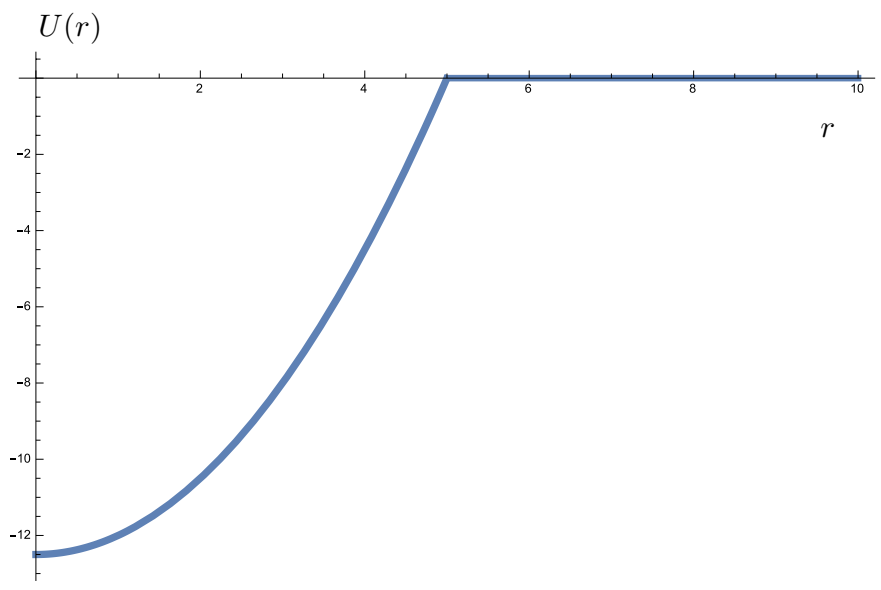

Figure 4.1: Harmonic potential with $\mu=\omega=1$ and $d=5$.

where $\mathcal{N}$ is a normalization constant, ${ }_{1} F_{1}$ the Kummer confluent hypergeometric function of the first kind $\left[122\right.$ and $Y_{l m}(\theta, \phi)$ are the usual spherical harmonics [122]. The corresponding eigenvalues are then given by,

$$
\varepsilon_{n l}=\hbar \omega\left(2 n+l+\frac{3}{2}\right)+\bar{U}(0)
$$

where $n$ is the radial quantum number.

Denoting by $n_{0}$ and $l_{0}$ the quantum number corresponding to the highest energy state of the well, i.e. $\mathcal{E}_{n_{0} l_{0}} \simeq 0$, we find that

$$
\bar{U}(0) \simeq-\hbar \omega\left(2 n_{0}+l_{0}+\frac{3}{2}\right),
$$

which, upon assuming $d \simeq \lambda_{\mathrm{G}}$, yields

$$
\omega \simeq \frac{2}{\lambda_{\mathrm{G}}}\left(2 n_{0}+l_{0}+\frac{3}{2}\right) .
$$

Thus, we conclude that

$$
\bar{U}(r)=2 \epsilon_{\mathrm{G}}\left(2 n_{0}+l_{0}+\frac{3}{2}\right)^{2} \frac{r^{2}-\lambda_{\mathrm{G}}^{2}}{\lambda_{\mathrm{G}}^{2}},
$$

and the corresponding eigenvalues are then given by

$$
\varepsilon_{n l} \simeq-2 \epsilon_{\mathrm{G}}\left(2 n_{0}+l_{0}+\frac{3}{2}\right)\left[2\left(n_{0}-n\right)+\left(l_{0}-l\right)\right],
$$

provided that $n \leq n_{0}$ and $l \leq l_{0}$. 


\subsubsection{Hayward spacetime}

One of the most famous examples of regular black holes 135 142 in semiclassical gravity is given by the Hayward spacetime [143], defined by the line element

$$
\mathrm{d} s^{2}=-f(r) \mathrm{d} v^{2}+2 \mathrm{~d} v \mathrm{~d} r+r^{2} \mathrm{~d} \Omega^{2}, \quad f(r)=1-\frac{2 G_{\mathrm{N}} M r^{2}}{r^{3}+2 G_{\mathrm{N}} M \ell^{2}},
$$

where $v$ denotes the advanced time coordinate, $r$ is the areal radius, $\mathrm{d} \Omega^{2}=\mathrm{d} \theta^{2}+\sin ^{2} \theta \mathrm{d} \varphi^{2}$, $M$ is the ADM mass of the system and $\ell$ denotes an UV cut-off responsible for the singularity resolution.

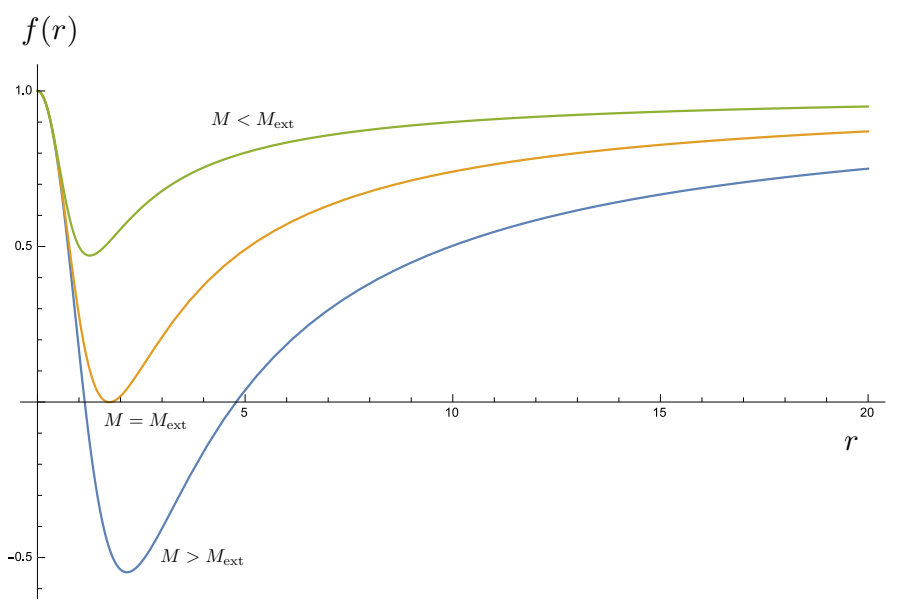

Figure 4.2: Hayward's metric function $f(r)$ for different values of the ADM mass $M$, keeping $\ell$ fixed.

It is also worth noting that

$$
f(r) \stackrel{r \rightarrow \infty}{\sim} 1-\frac{2 G_{\mathrm{N}} M}{r}, \quad f(r) \stackrel{r \rightarrow 0}{\sim} 1-\frac{r^{2}}{\ell^{2}}
$$

namely, we recover Schwarzschild for large $r$ whereas the central singularity gets removed by a de Sitter-like core, see 140,143.

The horizon structure is controlled by the inequality

$$
0 \leq \frac{3 \sqrt{3}}{4 G_{\mathrm{N}}} \ell \leq M
$$

and the horizons are then given by

$$
R_{\mathrm{H}}^{(j)}=\frac{4 G_{\mathrm{N}} M}{3} \cos \left[\frac{1}{3} \arccos \left(1-\frac{27 \ell^{2}}{8 G_{\mathrm{N}}^{2} M^{2}}\right)+\frac{2 \pi j}{3}\right]+\frac{2 G_{\mathrm{N}} M}{3}, \quad j=2,3
$$


from which it is easy to see that $R_{\mathrm{H}}^{(2)} \leq R_{\mathrm{H}}^{(3)}$, that allows us to make the identifications $R_{\mathrm{H}}^{(<)} \equiv R_{\mathrm{H}}^{(2)}$ and $R_{\mathrm{H}}^{(>)} \equiv R_{\mathrm{H}}^{(3)}$ for the inner and outer horizons, respectively. Besides, it is worth noting that the extremal configuration is reached when $R_{\mathrm{H}}^{(<)}=R_{\mathrm{H}}^{(>)}$and corresponds to a radius $R_{\mathrm{H}, \mathrm{ext}}=4 G_{\mathrm{N}} M / 3$ (see also Figure 4.2 ).

If we then consider a very small regular core, namely $\ell \ll G_{\mathrm{N}} M$, one can easily find

$$
R_{\mathrm{H}}^{(<)} \simeq \ell+\frac{\ell^{2}}{4 G_{\mathrm{N}} M}, \quad R_{\mathrm{H}}^{(>)} \simeq 2 G_{\mathrm{N}} M-\frac{\ell^{2}}{2 G_{\mathrm{N}} M} .
$$

Turning now our attention to the corpuscular model, let us prepare a bound state of $N$ toy gravitons held together by a collective harmonic potential (4.70) with $d \simeq \lambda_{\mathrm{G}} \simeq$ $\sqrt{N} \ell_{\mathrm{P}}$. With these assumptions one finds that the wave-function of each toy graviton in the marginally bound state can be expressed as

$$
\phi_{0}(r) \simeq\left(\frac{4 n_{0}+3}{\pi N \ell_{\mathrm{P}}^{2}}\right)^{3 / 4} \exp \left[-\left(2 n_{0}+\frac{3}{2}\right) \frac{r^{2}}{N \ell_{\mathrm{P}}^{2}}\right],
$$

since the total angular momentum of the Hayward space vanishes and we are therefore allowed to limit ourselves to the case $l=l_{0}=0$, for sake of simplicity. Thus, assuming the Hayward black hole to be the result of (sourced by) a coherent state of these toy gravitons [81, we have that the quantum state of the source, within the framework of the HQM, can therefore be approximated by

$$
\left|\Psi_{\mathrm{S}}\right\rangle \simeq \bigotimes_{i=1}^{N}\left|\phi_{0}^{(i)}\right\rangle=\left[\left|\phi_{0}\right\rangle\right]^{N}
$$

with $\left\langle r \mid \phi_{0}\right\rangle=\phi_{0}(r)$. Let us also remark that (4.84) is an eigenstate of the total Hamiltonian $\widehat{H}$, i.e.

$$
\left\langle\Psi_{\mathrm{S}}|\widehat{H}| \Psi_{\mathrm{S}}\right\rangle=M \simeq N \epsilon_{\mathrm{G}} .
$$

We can now compute the probability $P_{\mathrm{BH}}(\ell ; N)$ for this $N$-particle state to be a black hole. First, following Definition [6, it is easy to see that

$$
P_{\mathrm{S}}\left(r_{1}<R_{\mathrm{H}}, \ldots, r_{N}<R_{\mathrm{H}}\right)=\prod_{i=1}^{N} P_{\mathrm{S}}\left(r_{i}<R_{\mathrm{H}}\right) .
$$

Besides, we can approximate the total probability density, for the outer horizon, by (see [81)

$$
\mathcal{P}_{\mathrm{H}}^{(>)}\left(R_{\mathrm{H}}\right) \simeq \delta\left(R_{\mathrm{H}}-\left\langle\widehat{R}_{\mathrm{H}}^{(>)}\right\rangle\right),
$$

which is a direct consequence of the fact that we are interested in black holes of astrophysical size, i.e. $N \gg 1$, and thus the uncertainty over the horizon radius is negligible. Note that, using the scaling laws (3.45), we have that

$$
\left\langle\widehat{R}_{\mathrm{H}}^{(>)}\right\rangle \simeq 2 \sqrt{N} \ell_{\mathrm{P}}-\frac{\ell^{2}}{2 \sqrt{N} \ell_{\mathrm{P}}},
$$


in the limit $\ell \ll 2 G_{\mathrm{N}} M \simeq 2 \sqrt{N} \ell_{\mathrm{P}}$. We invite the interested reader to refer to 81 for a precise construction of $\widehat{R}_{\mathrm{H}}^{(\lessgtr)}$, which however is not that different from the one presented in Section 3.1 .4 for the rotating case.

After some simple computations one finds that

$$
P_{\mathrm{BH}}(\ell ; N) \simeq \prod_{i=1}^{N} P_{\mathrm{S}}\left(r_{i}<\left\langle\widehat{R}_{\mathrm{H}}^{(>)}\right\rangle\right) \simeq\left[P_{\mathrm{S}}\left(r<\left\langle\widehat{R}_{\mathrm{H}}^{(>)}\right\rangle\right)\right]^{N},
$$

that ultimately leads to

$$
\begin{aligned}
P_{\mathrm{BH}}(\ell ; N) \simeq & {\left[\frac{2}{\sqrt{\pi}} \gamma\left(\frac{3}{2}, 4\left(4 n_{0}+3\right)\right)\right]^{N-1} \times } \\
& {\left[\frac{2}{\sqrt{\pi}} \gamma\left(\frac{3}{2}, 4\left(4 n_{0}+3\right)\right)-\frac{8\left(4 n_{0}+3\right)^{3 / 2}}{\sqrt{\pi} N} e^{-4\left(4 n_{0}+3\right)} \frac{\ell^{2}}{\ell_{\mathrm{P}}^{2}}\right] }
\end{aligned}
$$

with

$$
\gamma(s, x)=\int_{0}^{x} \mathrm{~d} t t^{s-1} e^{-t}
$$

denoting the lower-incomplete Euler gamma function [122].

Notice that, if we set $\ell=0$ in 4.78 , we recover the Schwarzschild black hole. Thus, the quantity $\sigma(\ell ; N)=\left|P_{\mathrm{BH}}(0 ; N)-P_{\mathrm{BH}}(\ell ; N)\right|$ measures the breaking of a Schwarzschildlike black hole configuration due to quantum gravitational effects, implemented by the UV cut-off. Nonetheless, it was shown in [81] that $\sigma(\ell ; N) \ll 1$ in the considered regime, i.e. $\ell / \ell_{\mathrm{P}} \ll M / m_{\mathrm{P}} \sim \sqrt{N}$, for all fixed value of $n_{0}$ and $\forall N \gg 1$.

Turning our attention to the inner horizon, implementing a similar procedure to the one discussed above, one finds

$$
P_{\mathrm{IH}}(\ell ; N) \simeq\left\{\frac{2}{\sqrt{\pi}} \gamma\left[\frac{3}{2}, \frac{4 n_{0}+3}{N}\left(\frac{\ell}{\ell_{\mathrm{P}}}+\frac{\ell^{2}}{4 \sqrt{N} \ell_{\mathrm{P}}^{2}}\right)^{2}\right]\right\}^{N}
$$

This implies that

$$
\lim _{N \rightarrow \infty} P_{\mathrm{IH}}(\ell ; N)=0,
$$

for all fixed values of $n_{0}$. This means that, in the corpuscular picture, the formation of a non-singular black hole of the Hayward-type is rather unlikely.

\subsubsection{Kerr spacetime}

The case of spinning (chargeless) black holes, defined in terms of the line element 3.33 and with horizons given by (3.34), is a bit more involved at the quantum mechanical level [79].

Let us consider a Kerr black hole, of ADM mass $M$ and total angular momentum $J$, sourced by a system of marginally bound toy gravitons held together by an harmonic 
potential, in the framework of the quantum $N$-portrait of black holes. Hence, we can model the quantum state of the system as

$$
\left|\Psi_{\mathrm{S}}\right\rangle \equiv|M J\rangle=\bigotimes_{\alpha=1}^{N}|g\rangle_{\alpha}
$$

where $|g\rangle$ denotes the wave-function of each constituent toy graviton. Besides, it is also worth recalling that $\langle\widehat{H}\rangle \equiv\left\langle\Psi_{\mathrm{S}}|\widehat{H}| \Psi_{\mathrm{S}}\right\rangle=M \simeq N \epsilon_{\mathrm{G}}$.

As in Section 4.4.2, we can employ the harmonic potential 4.70, with $d \simeq \lambda_{\mathrm{G}} \simeq$ $\sqrt{N} \ell_{\mathrm{P}}$, to model the collective interaction that keeps the "condensate" together. Since the spacetime has a non-vanishing angular momentum, we are not allowed to simplify our discussion by ignoring the contribution of the quantum numbers $l$ and $m$, as we did in the Hayward case. Nonetheless, for the sake of simplicity, let us restrict ourselves to $n=n_{0}=0, l=l_{0}=2$ and $m= \pm 2$, from which we get

$$
\langle r, \theta, \phi \mid g\rangle=\psi_{02 \pm 2}\left(r, \theta, \phi ; \lambda_{\mathrm{G}}\right)=\frac{4}{\sqrt{15} \pi^{1 / 4} \lambda_{\mathrm{G}}^{7 / 2}} r^{2} \exp \left(-\frac{r^{2}}{2 \lambda_{\mathrm{G}}^{2}}\right) Y_{2 \pm 2}(\theta, \phi) \text {. }
$$

If we then denote by $N_{+}$the number of constituents with $m=+2$ (spin-up) and by $N_{-}=N-N_{+}$the number of constituents with $m=-2$ (spin-down), then assuming $N_{+} \geq N_{-}$one finds that

$$
\langle\widehat{J}\rangle \equiv\left\langle\Psi_{\mathrm{S}}\left|\widehat{J}^{2}\right| \Psi_{\mathrm{S}}\right\rangle=4\left(N_{+}-N_{-}\right)\left(N_{+}-N_{-}+1 / 2\right) m_{\mathrm{P}}^{2}=4 L^{2} N^{2} m_{\mathrm{P}}^{2},
$$

with

$$
L^{2}:=\left(\frac{N_{+}}{N}-\frac{N_{-}}{N}\right)\left(\frac{N_{+}}{N}-\frac{N_{-}}{N}+\frac{1}{2 N}\right) .
$$

In the limit $N \gg 1$, together with the assumption $N_{+} \geq N_{-}$(with $N_{+}=N_{-}$representing the non-rotating case), if we define $n_{+}:=N_{+} / N$ it is easy to see that $L^{2} \simeq\left(2 n_{+}-1\right)^{2}$, which implies

$$
\langle\widehat{J}\rangle \simeq 4\left(2 n_{+}-1\right)^{2} N^{2} m_{\mathrm{P}}^{2},
$$

in the large $N$ limit. Hence, the expectation values for the inner and outer horizons, as functions of $N$ and $n_{+}$, are then given by

$$
\left\langle\widehat{R}_{\mathrm{H}}^{( \pm)}\right\rangle=N \ell_{\mathrm{P}} \frac{\epsilon_{\mathrm{G}}}{m_{\mathrm{P}}}\left(1 \pm \sqrt{1-4 \frac{L^{2} m_{\mathrm{P}}^{4}}{N^{2} \epsilon_{\mathrm{G}}^{4}}}\right)
$$

see 79 and Section 3.1 .4 for a precise construction of $\widehat{R}_{\mathrm{H}}^{( \pm)}$.

Now, following the corpuscular picture, it is interesting to analyse the implications coming from assuming that all the constituents of our system lie inside the outer horizon $R_{\mathrm{H}}^{(+)}$. Thus, defining the parameter,

$$
\gamma\left(n_{+}, N\right):=\frac{\left\langle\widehat{R}_{\mathrm{H}}^{(+)}\right\rangle}{2 \lambda_{\mathrm{G}}}
$$


after some cumbersome manipulations we find (see [79]),

$$
P_{<}^{(+)}\left(r<\left\langle\widehat{R}_{\mathrm{H}}^{(+)}\right\rangle\right) \equiv P_{(+)}(\gamma)=\operatorname{erf}(2 \gamma)-\frac{4}{15 \sqrt{\pi}} \gamma\left(64 \gamma^{4}+40 \gamma^{2}+15\right) e^{-4 \gamma^{2}}
$$

by means of which we can compute,

$$
P_{\mathrm{BH}}\left(n_{+}, N\right)=\prod_{\alpha=1}^{N} P_{<}^{(+)}\left(r_{\alpha}<\left\langle\widehat{R}_{\mathrm{H}}^{(+)}\right\rangle\right)=\left[P_{(+)}(\gamma)\right]^{N},
$$

assuming $\mathcal{P}_{\mathrm{H}(+)}\left(R_{\mathrm{H}}\right)=\delta\left(R_{\mathrm{H}}-\left\langle\widehat{R}_{\mathrm{H}}^{(+)}\right\rangle\right)$, following the same reasoning of Section 4.4.2.

From this study we can conclude that a Kerr-like outer horizon is rather likely to form for a spinning corpuscular black hole, see [81] for details on the numerical analysis. However, if we repeat this argument for the inner horizon we find that its formation appears to be rather unlikely, particularly for large black holes.

\subsection{Lower dimensional corpuscular gravity}

It is a well known fact that gravity behaves in a very weird way if we consider lower dimensional spacetimes, i.e. $(1+d)$-spacetimes with spatial dimension $d<3$. The main motivation for investigating these kind of spaces is given by the fact that all approaches to quantum gravity predict a sort of effective dimensional reduction at very high energy, see e.g. $144-150$.

One of the key results coming from the dimensional reduction approach to gravity is that the gravitational constant gains a peculiar behaviour [147], namely

$$
G_{d}=2 \pi^{1-\frac{d}{2}} \Gamma\left(\frac{d}{2}\right) \frac{\ell_{d}^{d-1}}{\hbar} \simeq \frac{\ell_{d}^{d-1}}{\hbar}
$$

where $G_{d}$ and $\ell_{d}$ are, respectively, the gravitational constant and the Planck length for a $(1+d)$-dimensional spacetime. Specifically, it is worth remarking that $G_{3}=G_{\mathrm{N}}$ and $\ell_{3}=\ell_{\mathrm{P}}$.

By inspection of 4.101 it is trivial to notice the particularly strange behaviour of $G_{d}$ when $d=1$, i.e.

$$
G_{1} \simeq \frac{1}{\hbar}
$$

in other words, $G_{1}$ is dual to $\hbar$. Besides, it is found that the $d=1$ analogue to the Schwarzschild solution [144 is given by the line element

$$
\mathrm{d} s^{2}=-\left(1-2 G_{1} M r\right) \mathrm{d} t^{2}+\left(1-2 G_{1} M r\right)^{-1} \mathrm{~d} r^{2},
$$


which is obtained by solving Einstein's field equations in $(1+1)$-dimensions and where $M$ denotes the mass of the system. Form (4.103) it is now easy to compute the location of the horizon, which is given by

$$
R_{\mathrm{H} 1}=\frac{1}{2 G_{1} M} .
$$

It is mostly agreed upon that quantum mechanical laws should be (somehow) independent on the dimension of the spacetime in which they live in. Thus, quantities like the de Broglie or Compton wavelengths represent length scales which are typical of the object at hand and are independent of the nature of the spacetime. Now, recalling that the typical quantum size of an object of energy $E$, as stressed many times in this work, is given by $\lambda_{E} \simeq \hbar / E$, then we can immediately observe that,

$$
R_{\mathrm{H} 1}=\frac{1}{2 G_{1} M}=\frac{\hbar}{2 M} \simeq \lambda_{M} .
$$

This means that, in $d=1$, the typical quantum size of a black hole of mass $M$ and the size of the horizon are essentially indistinguishable features of the system. This statement is further supported by the fact that $d=1$ black holes do not admit a generalized uncertainty principle (GUP, see e.g. $771,151,152]$ ) [76]. The metric (4.104) turns out to be rather peculiar also from a thermodynamic perspective, indeed one finds that

$$
T_{\mathrm{H} 1} \sim M, \quad S_{\mathrm{BH} 1} \sim \log \left(\frac{M}{m_{\star}}\right),
$$

where $T_{\mathrm{H} 1}$ and $S_{\mathrm{BH} 1}$ denote the Hawking temperature and the entropy of the black hole, while $m_{\star}$ denotes an emerging mass scale (not necessarily related to $m_{\mathrm{P}}$ ). This clearly shows that the scaling of the entropy is not holographic.

In the (typical, $d=3$ ) corpuscular model of black holes we find that each graviton in the coherent state has a typical energy $\epsilon_{\mathrm{G}}$, while the total energy stored in the gravitational field (within a sphere of radius $R$ ) takes the form

$$
U_{3}(R) \simeq \frac{G_{\mathrm{N}} M^{2}}{R} \equiv \frac{G_{3} M^{2}}{R},
$$

with $\lambda_{\mathrm{G}}(R) \simeq \hbar / \epsilon_{\mathrm{G}}(R) \simeq R$. Hence, we can estimate the number of gravitons in the bound state as

$$
N_{3}=\frac{U_{3}(R)}{\epsilon_{\mathrm{G}}(R)} .
$$

If we then take $R_{3} \rightarrow 2 G_{\mathrm{N}} M$ we get the scaling law $\epsilon_{\mathrm{G}} \simeq m_{\mathrm{P}} / \sqrt{N}$, which is verified only in the critical limit (i.e. when the black hole has formed).

Now, since $\epsilon_{\mathrm{G}}$ is the typical quantum energy scale for the gravitons in the condensate, it should be insensitive to an effective dimensional reduction of the space. Thus, let us try 
to see what happens if we consider the $d=1$ black hole in (4.104), within the corpuscular picture (see $153 \mid$ ). In the $d=1$ case we find that the total gravitational binding energy reads,

$$
U_{1}(R) \simeq G_{1} M^{2} R .
$$

Thus, recalling that $\epsilon_{\mathrm{G}}(R) \simeq \hbar / R$, we find

$$
N_{1}=\frac{U_{3}(R)}{\epsilon_{\mathrm{G}}(R)} \simeq \frac{M^{2} R^{2}}{\hbar^{2}} .
$$

If we then take the limit $R \rightarrow R_{\mathrm{H} 1}=\left(2 G_{1} M\right)^{-1}$, we find $N_{1} \simeq 1$. Besides, if we compare this result with the $d=3$ case, namely $N_{3} \simeq M^{2} / m_{\mathrm{P}}^{2}$, we find that $N_{3} \sim 1$ only if $M \sim m_{\mathrm{P}}$. This suggests that $d=1$ black holes are strictly Planckian objects in the corpuscular picture and, since they should be made of roughly a single constituent, they are also intrinsically quantum. 


\section{Chapter 5}

\section{Corpuscular cosmology}

So far we have discussed how the classicalization scenario can help us understanding the strong gravity regime, within the frameworks of high energy scattering processes and the gravitational collapse. Specifically, we have been able to provide a fully quantum (effective) theory of black holes in terms of condensates of gravitons on the verge of a quantum phase transition. It is now interesting to see that, if we extend this simple picture to larger scales, one recovers some known results concerning the physics of the early Universe and galactic dynamics.

In this Chapter we will first present a formulation of the slow-roll inflationary scenario based on the corpuscular model, first presented in |154 and further discussed in 155, 156 within the framework of modified theories of gravity. The key feature of the latter, in particular, is that the expected exponential expansion is now a direct consequence of the graviton self-interaction, which is responsible for balancing the various contributions in the Hamiltonian constraint 155, 156], rather than coming from an additional condensate of some exotic degrees of freedom (inflaton), assisting both the expansion and the graviton depletion 154. Then, following a similar philosophy and assuming the existence of a cosmological condensate of gravitons, we will investigate the emergence of the observed dynamics at galactic scales. Precisely, we will describe the emergence of Modified Newtonian dynamics (MOND) effects, at galactic scales, as the reaction of the cosmological condensate to the local presence of baryonic matter [157,158.

\subsection{Corpuscular de Sitter space}

As fist step toward a corpuscular description of the large scale structure of the Universe one has to provide a fundamental justification for the observed cosmic expansion. The $\Lambda \mathrm{CDM}$ model (i.e. $\Lambda$ cold dark matter model) represents the reference picture for modelling the Universe, at large scales, and entails only contributions from baryonic matter, radiation, dark energy and cold dark matter. Specifically, this model is based on few cardinal assumptions [159]: the gravitational interaction is properly described in terms of Einstein's field equation with cosmological constant $\Lambda$, at cosmological scales; the dominant forms 
of energy and matter are distributed in a uniform and homogeneous way at these scales; matter and radiation are accurately described as perfect barotropic fluids. Under these assumptions, it is easy to see (see e.g. $|159|$ ) that the geometry of our Universe, at large scales, is well approximated by a Friedman-Lemaître-Robertson-Walker (FLRW) metric, i.e.

$$
\mathrm{d} s^{2}=-\mathrm{d} t^{2}+a^{2}(t)\left(\frac{\mathrm{d} r^{2}}{1-k r^{2}}+r^{2} \mathrm{~d} \Omega^{2}\right)
$$

where $t$ denotes the cosmic time, $k$ is the curvature parameter of the 3 -dimensional spacelike slices and $a(t)$ is the well known scale factor. If we then require this spacetime to be a solution of the Einstein equations with cosmological constant, and sourced by a perfect fluid - accounting for ordinary matter (baryonic), cold dark matter (non-baryonic) and radiation - we find that this set of equations reduces to the Friedman equations,

$$
\begin{aligned}
H^{2} & \equiv\left(\frac{\dot{a}}{a}\right)^{2}=\frac{8 \pi G_{\mathrm{N}}}{3} \rho-\frac{k}{a^{2}}, \\
\frac{\ddot{a}}{a} & =-\frac{4 \pi G_{\mathrm{N}}}{3}(\rho+3 p)
\end{aligned}
$$

where the dot represents the total time derivative, $H:=\dot{a} / a$ is the Hubble parameter, and $\rho$ and $p$ are, respectively, the energy density and pressure of the perfect barotropic (i.e. $\rho=\rho(p))$ fluid. It is now worth noting that the first Friedman equation (5.2) is not an evolution equation, since it involves only first order derivatives. Indeed, it can be shown (see e.g. 160|) that it implements the Hamiltonian constraint of gravity or, more precisely, (5.2) is the Hamiltonian constraint for the Einstein-Hilbert action with cosmological constant restricted to the class of maximally symmetric spacetimes.

Let us now consider the case of a flat Universe $(k=0$, flat $3 \mathrm{~d}$ slices) filled only with dark energy which, in the standard model of cosmology, is fully accounted for by the cosmological constant term $\Lambda$. The corresponding energy density is then given by

$$
\rho_{\Lambda}=-p_{\Lambda}=\frac{\Lambda}{8 \pi G_{\mathrm{N}}}
$$

for which we have that the Friedman equations reduce to

$$
\begin{aligned}
H^{2} & =\frac{\Lambda}{3}, \\
\ddot{a} & =\frac{\Lambda}{3},
\end{aligned}
$$

which are ultimately solved by

$$
a(t) \propto \exp \left(\sqrt{\frac{\Lambda}{3}} t\right)
$$


that describes an exponentially expanding Universe. The latter is known in the literature as the de Sitter solution. We can thus infer that in an era in which the dark energy is the dominating component, the spacetime undergoes a phase of exponential expansion. The existence of such a phase in the evolution of the Universe, known as cosmic inflation [21-24], is of paramount importance since it provides a natural solution for several issues like the horizon problem, the flatness problem and absence of stable magnetic monopoles. However, since our Universe is currently expanding, but not at an exponential rate, cosmic inflation should involve a mechanism that makes the inflationary scenario last long enough to solve all the problems mentioned above and then drives the system out of this phase, namely it should involve a sort of graceful exit.

We shall come back to cosmic inflation and its relation to the corpuscular model in Section 5.2. For the time being, let us just focus on the de Sitter Universe and see how we can relate it to the classicalization picture. In FLRW cosmology, the functional expression of the Hamiltonian constraint is proportional to the Hamiltonian through the lapse function $N(t)$, namely $H=N \mathcal{H}$ with $\mathcal{H}=0$ denoting the constraint (see e.g. $[160]$ ). One can then separate the contributions coming from the gravitational and matter parts of $\mathcal{H}$, thus rewriting the constraint as

$$
\mathcal{H}=\mathcal{H}_{\mathrm{G}}+\mathcal{H}_{\mathrm{M}}=0
$$

in full analogy with the discussion in Section 4.2, In the corpuscular picture $\mathcal{H}_{\mathrm{G}}$ will then represent the energy content due to the purely gravitational part of the system, whereas $\mathcal{H}_{\mathrm{M}}$ will give us information on the matter contribution to the total energy.

Now, going back to the first Friedman equation for the de Sitter space (5.5), if we integrate both sides of the latter over the volume within a sphere of the Hubble radius, defined as $L_{\Lambda}:=1 / H_{\Lambda}=\sqrt{3 / \Lambda}$, one immediately finds that the constraint equation reduces to

$$
L_{\Lambda} \simeq G_{\mathrm{N}} M_{\Lambda}, \text { with } M_{\Lambda} \simeq L_{\Lambda}^{3} \rho_{\Lambda}
$$

i.e. we recover a relation between the Hubble radius of de Sitter and the "energy" contribution of the cosmological constant inside an Hubble sphere that resembles the one connecting the gravitational radius and the ADM mass for a Schwarzschild black hole. We can then try to draw an analogy between the quantum $N$-portrait for black holes and the fundamental description of the de Sitter spacetime [154. Specifically, let us assume that the graviton self-interaction can induce a condensation of $N_{\Lambda}$ quanta of typical wavelength $\lambda_{\mathrm{G}} \simeq L_{\Lambda}$. These assumptions ultimately lead to the typical corpuscular scaling laws (3.45), as discussed in 154. An alternative derivation of these laws can be obtained by means of a simple energy balance argument, analogous to the one presented in Section 4.2 for the gravitational collapse and based on the Hamiltonian constraint of Cosmology. Precisely, one has that 155

$$
\mathcal{H}=\mathcal{H}_{\mathrm{G}}=0 .
$$


This is due to the fact that we are considering a Universe filled only with dark energy that, according to our assumptions, should be the result of a condensate of self-interacting gravitons. Hence we have that $\mathcal{H}_{\mathrm{M}}=0$.

Now, the coherent state resulting from the condensation of $N_{\Lambda}$ (assumed to be large) gravitons gives rise to a Newtonian potential energy, as discussed in Section 4.1 and 4.2 . that reads

$$
U_{\mathrm{N}}=M_{\Lambda} V_{\mathrm{N}}\left(L_{\Lambda}\right) \simeq-N_{\Lambda} \epsilon_{\mathrm{G}} \simeq-N_{\Lambda} \frac{\hbar}{L_{\Lambda}},
$$

where $\epsilon_{\mathrm{G}} \simeq \hbar / \lambda_{\mathrm{G}}$ is the typical energy of the constituent gravitons, as usual. Besides, due to the self-interacting nature of these quanta, each constituent will then interact with the collective state giving rise to a contribution similar (in form) to the post-Newtonian energy, i.e.

$$
U_{\mathrm{PN}} \simeq-N_{\Lambda} \epsilon_{\mathrm{G}} V_{\mathrm{N}} \simeq N_{\Lambda}^{3 / 2} \frac{m_{\mathrm{P}} \ell_{\mathrm{P}}^{2}}{L_{\Lambda}^{2}}
$$

Thus the Hamiltonian constraint can be rewritten as

$$
\mathcal{H}=\mathcal{H}_{\mathrm{G}} \simeq U_{\mathrm{N}}+U_{\mathrm{PN}}=0
$$

that yields,

$$
L_{\Lambda} \simeq \sqrt{N_{\Lambda}} \ell_{\mathrm{P}}
$$

Besides, recalling that $\lambda_{\mathrm{G}} \simeq \hbar / \epsilon_{\mathrm{G}} \simeq L_{\Lambda}$ and that $M_{\Lambda} \simeq L_{\Lambda} / G_{\mathrm{N}}$, it is easy to infer that

$$
\epsilon_{\mathrm{G}} \simeq \frac{m_{\mathrm{P}}}{\sqrt{N_{\Lambda}}} \equiv \epsilon_{\Lambda}, \text { and } M_{\Lambda} \simeq \sqrt{N_{\Lambda}} m_{\mathrm{P}}
$$

which represent the typical corpuscular scaling laws for a de Sitter space generated by the condensation of $N_{\Lambda}$ soft gravitons with $\lambda_{\mathrm{G}} \simeq L_{\Lambda}$.

It is now interesting to observe that the de Sitter space can also be obtained as an exact solution of a modified theory of gravity $161-168$. Indeed, if we consider

$$
S=\frac{1}{16 \pi G_{\mathrm{N}}} \int \mathrm{d}^{4} x \sqrt{-\operatorname{det} g} f(R), \quad \text { with } f(R)=\gamma \ell_{\mathrm{P}}^{2} R^{2},
$$

with $\gamma$ a dimensionless constant, one finds that the corresponding evolution equation, in a FLRW background, read

$$
12 R H^{2}=R^{2}-12 H \dot{R},
$$

which is solved by $a(t)=\exp \left(H_{\Lambda} t\right)$ with $H_{\Lambda}=\sqrt{\Lambda / 3}$. If we then expand (5.17) around the de Sitter solution $a(t)=\exp \left(H_{\Lambda} t\right)$, we find that 5.17 reduces to $H_{\Lambda}^{2}=\Lambda / 3$, as 
expected. Thus, integrating separately $H_{\Lambda}^{2}$ and $\Lambda / 3$ over the volume within a sphere of the Hubble radius $L_{\Lambda}$ we find that

$$
L_{\Lambda}^{3} H_{\Lambda}^{2} \simeq L_{\Lambda}^{3} \frac{\Lambda}{3} \simeq L_{\Lambda}
$$

It is now interesting to see that, upon replacing the scaling laws 5.14 and 5.14 into (5.11) and 5.12) one finds,

$$
U_{\mathrm{N}}=-\frac{m_{\mathrm{P}} L_{\Lambda}}{\ell_{\mathrm{P}}}, \quad U_{\mathrm{PN}}=\frac{m_{\mathrm{P}} L_{\Lambda}}{\ell_{\mathrm{P}}},
$$

that, once compared with (5.18), suggest us the following naive (but general) identifications,

$$
U_{\mathrm{N}}=-\frac{L_{\Lambda}^{3} H_{\Lambda}^{2}}{G_{\mathrm{N}}}, \quad U_{\mathrm{PN}}=\frac{L_{\Lambda}^{3} \Lambda}{3 G_{\mathrm{N}}} .
$$

These relations will serve as starting point to build a connection between the corpuscular model and Starobinsky's inflation, see Section 5.2.

Remark. Notice that in (5.16) we have changed the global sign of the action with respect to (2.6). This does not really change much if no matter contribution is considered. Indeed, these two representations are, in general, equivalent provided that we change the sign in the functional definition of the energy-momentum tensor.

\subsection{Slow-roll inflation and domestic inflaton}

In the previous Section have discussed how the de Sitter space can emerge in the corpuscular theory of gravity. However, it is rather clear that such a spacetime cannot suitably describe the whole dynamical history of our Universe. This is due to the fact that experimental observations clearly show that our Universe is indeed expanding, but with a much slower rate than the exponential one $[169,170 \mid$. Nonetheless, an initial phase of exponential expansion is the key to resolve many conceptual issues of modern cosmology [21,22]. Yet, in order to recover the correct picture of the Universe, at late times, one needs to find a mechanism that allows cosmic inflation to last long enough and then smoothly reduces the expansion rate of the spacetime. A widely accepted model that serves this purpose is the so called Starobinsky's model of inflation, or simply Starobinsky inflation [23, 24]. Specifically, this model of cosmic inflation is an $f(R)$ theory of gravity given by

$$
S=\frac{1}{16 \pi G_{\mathrm{N}}} \int \mathrm{d}^{4} x \sqrt{-\operatorname{det} g} f(R), \quad \text { with } f(R)=\alpha R+\gamma \ell_{\mathrm{P}}^{2} R^{2},
$$

where $\alpha, \gamma$ are two dimensionless constants. This action can be brought to the Einstein frame by means of a conformal transformation, namely $\bar{g}_{\mu \nu}=f^{\prime}(R) g_{\mu \nu}$ with the prime denoting the derivative with respect to the argument, and reads

$$
\bar{S}=\int d^{4} x \sqrt{-\operatorname{det} \bar{g}}\left[\frac{\bar{R}}{16 \pi G_{\mathrm{N}}}-\frac{1}{2} \bar{g}^{\mu \nu} \partial_{\mu} \varphi \partial_{\nu} \varphi-V(\varphi)\right]
$$


where the new scalar field $\varphi$ is defined by

$$
\varphi=\sqrt{\frac{3}{16 \pi G_{\mathrm{N}}}} \ln f^{\prime}(R)=\sqrt{\frac{3 m_{\mathrm{P}}}{16 \pi \ell_{\mathrm{P}}}} \log \left(\alpha+2 \gamma \ell_{\mathrm{P}}^{2} R\right)
$$

and the corresponding potential reads

$$
V(\varphi)=\frac{f^{\prime}(R(\varphi)) R(\varphi)-f(R(\varphi))}{16 \pi G_{\mathrm{N}} f^{\prime}(R(\varphi))^{2}}=\frac{m_{\mathrm{P}}}{64 \pi \ell_{\mathrm{P}}^{3} \gamma}\left[1-\alpha \exp \left(-\sqrt{\frac{16 \pi \ell_{\mathrm{P}}}{3 m_{\mathrm{P}}}} \varphi\right)\right]^{2} .
$$

Now, it is easy to see that

$$
\lim _{\alpha \rightarrow 0} V(\varphi)=\lim _{\varphi \rightarrow \infty} V(\varphi)=\frac{m_{\mathrm{P}}}{64 \pi \ell_{\mathrm{P}} \gamma},
$$

namely, in the limit $\alpha \rightarrow 0$ we recover the expected de Sitter behaviour, since the theory reduces to the pure quadratic gravity scenario (5.16). However, when $\alpha$ gets greater that zero the system becomes unstable and tends to depart from the de Sitter Universe. From Figure 5.1 one can see that if we start from a de Sitter configuration, namely we are on the plateau of the potential, where quadratic gravity dominates, once we turn on the Einstein-Hilbert part of the action, namely $R$, we see that the system starts slowly rolling down toward the minimum. Now, it is worth noting that, when the system leaves the plateau, the Universe undergoes a phase transition, from a quadratic gravity phase to an Einstein-Hilbert one.

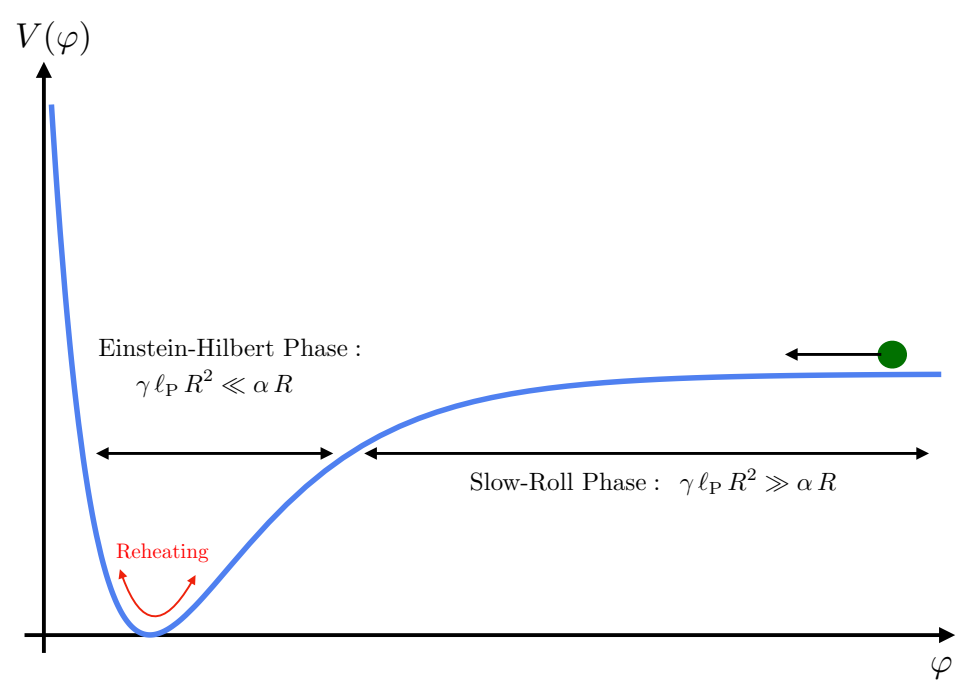

Figure 5.1: Starobinsky inflationary potential.

All these observations can be easily inferred from the equation of motion of the model, that for 5.21 reads

$$
6 \frac{\alpha}{\gamma \ell_{\mathrm{P}}^{2}} H^{2}+12 R H^{2}=R^{2}-12 H \dot{R}
$$


If we then consider a quasi-de Sitter ansatz, i.e. $H(t) \simeq H_{\Lambda}=1 / L_{\Lambda}$, one finds that the Hubble parameter is indeed slowly decreasing according to

$$
\dot{H} \simeq-\frac{\alpha}{\gamma \ell_{\mathrm{P}}^{2}},
$$

if $0<\alpha / \gamma \ll 1$. Recalling the definition of the first slow-roll parameter $\varepsilon$, i.e.

$$
\varepsilon:=-\frac{\dot{H}}{H^{2}} \sim G_{\mathrm{N}}\left(\frac{V^{\prime}(\varphi)}{V(\varphi)}\right)^{2},
$$

from the quasi-de Sitter ansatz, together with (5.27), one can easily find that

$$
\varepsilon \simeq \frac{\alpha L_{\Lambda}^{2}}{\gamma \ell_{\mathrm{P}}^{2}},
$$

which is required to satisfy $\varepsilon \ll 1$ on the plateau. However, at the end of inflation $\varepsilon \sim 1$ which implies

$$
\dot{H} \simeq-\frac{1}{L_{\Lambda}}, \quad \frac{\gamma}{\alpha} \simeq \frac{L_{\Lambda}^{2}}{\ell_{\mathrm{P}}^{2}} .
$$

Assuming that this initial quasi-de Sitter configuration can be thought of as a self-sustained marginally bound state of gravitons [155], with scaling laws as in (5.14) and (5.15), from 5.30 one can conclude that,

$$
\frac{\gamma}{\alpha} \simeq \frac{L_{\Lambda}^{2}}{\ell_{\mathrm{P}}^{2}} \simeq N_{\Lambda},
$$

which means that the coupling between ordinary and quadratic gravity, in a naive corpuscular perspective, is measured by the number of constituent gravitons. Besides, since $N_{\Lambda} \gg 1$ in order for classicalization to kick in, we also recover the expected condition $\gamma / \alpha \gg 1$. On top of this, using CMB data [171] one can even provide a very rough estimate of the number of constituents of the cosmological condensate, i.e. $N_{\Lambda} \simeq \gamma / \alpha \sim 10^{8}$.

Our aim is now to recover the same general results by means of a purely corpuscular argument. In Section 5.1 we described the emergence of the de Sitter space in quadratic gravity as a result of a corpuscular interpretation of the Hamiltonian constraint. If we do the same for the Einstein-Hilbert action $f(R)=\alpha R$ (in FLRW form) we find that the first Friedman equation is simply $H^{2}=0$. Then, taking profit of the identifications (5.20), one can easily conclude that the Hamiltonian constraint function for this scenario is simply given by $\mathcal{H} \sim U_{\mathrm{N}}$, namely it only contains the Newtonian contribution. Whereas, it is worth recalling that, in Section 5.1, we found that for $f(R)=\gamma \ell_{\mathrm{P}}^{2} R^{2}$ the Hamiltonian constraint function was given by $\mathcal{H} \sim U_{\mathrm{N}}+U_{\mathrm{PN}}$, or to be more specific, it also contains a "postNewtonian" contribution. Now, if we start with an initial phase of de Sitter expansion, then the system should approximately satisfy the energy balance condition

$$
\mathcal{H}_{\left(R^{2}\right)} \simeq U_{\mathrm{N}}+U_{\mathrm{PN}} \simeq 0
$$


corresponding to the effective metric action (5.16) of quadratic gravity. However, in order to accurately describe our Universe, we are required to include the contribution of ordinary (Einstein-Hilbert) gravity, for which we have $\mathcal{H}_{(R)} \simeq U_{\mathrm{N}}$. Thus, the full energy balance for our Universe is given by 155

$$
\mathcal{H} \simeq \alpha \mathcal{H}_{(R)}+\beta \mathcal{H}_{\left(R^{2}\right)} \simeq 0,
$$

where $\alpha>0$ can be viewed as the coefficient appearing in the Einstein-Hilbert part of the action (5.21) while $\beta>0$ is a dimensionless parameter, presumably of order one, used to keep track of the contribution of quadratic gravity.

In a stage where the departure from the de Sitter space is small, one can assume that the potentials take on a slightly more general form

$$
U_{\mathrm{N}} \simeq-\frac{L^{3} H^{2}}{G_{\mathrm{N}}}
$$

and

$$
U_{\mathrm{PN}} \simeq \frac{L^{3}}{G_{\mathrm{N}} L_{\Lambda}^{2}},
$$

where $L \simeq L_{\Lambda}$ is the new Hubble radius. Since 5.33 can be rewritten as

$$
\alpha U_{\mathrm{N}}+\beta\left(U_{\mathrm{N}}+U_{\mathrm{PN}}\right) \simeq 0
$$

taking profit of 5.34 and 5.35 one can conclude that,

$$
H^{2} \simeq \frac{\beta}{\alpha+\beta} \frac{1}{L_{\Lambda}^{2}}
$$

Besides, it is also worth noting that expanding around the de Sitter solution,

$$
H \simeq H_{\Lambda}+\delta t \dot{H}
$$

and recalling that $\delta t \simeq \lambda_{\mathrm{G}} \simeq L_{\Lambda}$, since constituent gravitons cannot be sensitive to shorter times, one can conclude that 155

$$
\dot{H} \simeq-\frac{\alpha}{\alpha+\beta} \frac{1}{L_{\Lambda}^{2}}
$$

which is precisely $(5.30)$, with the rather natural choice of parameters $\alpha /(\alpha+\beta) \sim 1$.

Now, it is interesting to see how it is possible to use the quantum depletion of the graviton condensate to provide some restriction of the parameter $\alpha$ appearing in the postNewtonian analysis. First, from (5.37) and (5.39) we have that

$$
\varepsilon=-\frac{\dot{H}}{H^{2}} \simeq \frac{\alpha}{\beta},
$$


where, again, $\beta=\mathcal{O}(1)$ positive dimensionless parameter used to keep track of $\mathcal{H}_{\left(R^{2}\right)}$. Thus, in this picture, the beginning of inflation corresponds to the condition $\alpha \ll 1$, while the end of this scenario is given by $\alpha \sim \beta \sim 1$. If we then include the effect of the depletion of gravitons out of the condensate [154], the time evolution of the number of constituent gravitons will be governed by

$$
\frac{\dot{N}_{\Lambda}}{N_{\Lambda}} \simeq \frac{\varepsilon}{L_{\Lambda}}\left(1-\frac{1}{N_{\Lambda} \varepsilon^{3 / 2}}\right)
$$

by means of which it is easy to see that

$$
-\frac{\dot{H}}{H^{2}} \simeq \frac{\ell_{\mathrm{P}} \dot{N}_{\Lambda}}{\sqrt{N_{\Lambda}}} \simeq \varepsilon\left(1-\frac{1}{N_{\Lambda} \varepsilon^{3 / 2}}\right)
$$

Similarly to what was observed in [154, the condition

$$
\varepsilon=\varepsilon_{\star} \simeq \frac{1}{N_{\Lambda}^{2 / 3}} \simeq\left(\frac{\ell_{\mathrm{P}}}{L_{\Lambda}}\right)^{4 / 3}
$$

tells us about the closer our system can get the de Sitter spacetime. In fact, the condition $\varepsilon=\varepsilon_{\star}$, in the corpuscular post-Newtonian picture of inflation, corresponds to $\alpha \simeq 0$, namely that only the quadratic gravity constraint survives $|155|$. More precisely, if we include the effects of depletion we get some precise restrictions on the value of $\alpha$, specifically

$$
\left(\ell_{\mathrm{P}} / L_{\Lambda}\right)^{4 / 3} \lesssim \alpha \lesssim 1
$$

To sum up, the key message coming from the post-Newtonian analysis of the corpuscular de Sitter space is that there is no need to introduce exotic matter to describe an eternally inflating Universe [155, 156. It is sufficient to consider a condensate of gravitons that mimics the Hamiltonian constraint emerging in quadratic gravity. In this picture, the exponential expansion is then given by the balance of the Newtonian and post-Newtonian energies. However, since our Universe is not expanding at an exponential rate, we have to introduce a contribution that brakes this balance in favor of ordinary gravity. It turns out that ordinary (Einstein-Hilbert) gravity itself, seen as a small perturbation of quadratic gravity during inflation, is the simplest thing that gets the job done at the corpuscular level. Thus we can conclude that Starobinsky inflation emerges naturally from the corpuscular model of gravity. In this respect, one could wonder why an intrinsically quantum scenario, like the corpuscular model, is able to faithfully reproduce the same dynamics predicted by a classical action like the one in (5.21). To address this matter we just need to recall the discussion in Section 2.3. Indeed, in the 1960s Utiyama and DeWitt [172] have proven that the (regularized) one-loop effective action for Einstein-Hilbert gravity contains contributions of higher order curvature terms. This implies that all higher order curvature terms in an $f(R)$-theory should carry a coupling constant that scales like a positive power of $G_{\mathrm{N}} \hbar$. This tells us that the Starobinsky action is, per se, intrinsically quantum and its 
effects reduce to the classical Einstein-Hilbert dynamics in the classical limit, namely for $\hbar \rightarrow 0$. That is why it is not surprising that a corpuscular interpretation of the Hamiltonian constraint coming from the Starobinsky model yields the same effective dynamics.

Let us then try to go a bit beyond this very simple argument, based on energy balances, by expanding the Starobinsky action over a generic curved background [156]. We are particularly interested in understanding what happens to the number and nature of the propagating degrees of freedom of the theory. For sake of simplicity, let us set $\alpha=1$ and $\gamma \ell_{\mathrm{P}}^{2}=m_{\mathrm{P}}^{2} \ell_{\mathrm{P}}^{2} / 6 M^{2}$ in 5.21), where $M$ is usually referred to as the mass of the inflaton. The general equations of motion for the Starobinsky model are given by

$$
R_{\mu \nu}-\frac{1}{2} g_{\mu \nu} R+\gamma \ell_{\mathrm{P}}^{2} R\left(2 R_{\mu \nu}-\frac{1}{2} g_{\mu \nu} R\right)+2 \gamma \ell_{\mathrm{P}}^{2}\left(g_{\mu \nu} \square-\nabla_{\mu} \nabla_{\nu}\right) R=0,
$$

where $\square \equiv g^{\mu \nu} \nabla_{\mu} \nabla_{\nu}$. If we then try to expand (5.44) around a fiducial curved background $\bar{g}_{\mu \nu}$, i.e. $g_{\mu \nu}=\bar{g}_{\mu \nu}+h_{\mu \nu}+\mathcal{O}\left(h_{\mu \nu}^{2}\right)$ with $\left|h_{\mu \nu}\right| \ll\left|\bar{g}_{\mu \nu}\right|$, we can further make the decomposition for $h_{\mu \nu}$,

$$
h_{\mu \nu}=h_{\mu \nu}^{\perp}+\ell_{\mathrm{P}}\left(\bar{\nabla}_{\mu} A_{\nu}^{\perp}+\bar{\nabla}_{\nu} A_{\mu}^{\perp}\right)+\frac{1}{3} \bar{g}_{\mu \nu} \varphi+\frac{\bar{\nabla}_{\mu} \bar{\nabla}_{\nu}}{\square} \chi
$$

with the bar denoting that the operators are computed over the background metric, while

$$
\bar{g}^{\mu \nu} h_{\mu \nu}^{\perp}=0, \quad \bar{\nabla}^{\mu} h_{\mu \nu}^{\perp}=0 \quad \text { and } \quad \bar{\nabla}^{\mu} A_{\mu}^{\perp}=0,
$$

separate the transverse traceless component $h_{\mu \nu}^{\perp}$ from the scalar and vector ones. Besides, one can also show 173,174 that,

$$
\begin{aligned}
h_{\mu \nu}^{\perp}= & h_{\mu \nu}-\frac{\bar{\nabla}_{\mu}}{\bar{\square}}\left(\bar{g}_{\nu \rho}-\frac{\bar{\nabla}_{\nu} \bar{\nabla}_{\rho}}{\bar{\square}}\right) \bar{\nabla}_{\sigma} h^{\rho \sigma}-\frac{\bar{\nabla}_{\nu}}{\bar{\square}}\left(\bar{g}_{\mu \rho}-\frac{\bar{\nabla}_{\mu} \bar{\nabla}_{\rho}}{\bar{\square}}\right) \bar{\nabla}_{\sigma} h^{\rho \sigma} \\
& -\frac{1}{3}\left(\bar{g}_{\mu \nu}-\frac{\bar{\nabla}_{\mu} \bar{\nabla}_{\nu}}{\bar{\square}}\right)\left(\bar{g}_{\rho \sigma}-\frac{\bar{\nabla}_{\rho} \bar{\nabla}_{\sigma}}{\bar{\square}}\right) h^{\rho \sigma}-\frac{\bar{\nabla}_{\mu} \bar{\nabla}_{\nu} \bar{\nabla}_{\rho} \bar{\nabla}_{\sigma}}{\bar{\square}} h^{\rho \sigma}, \\
A_{\mu}^{\perp}= & \frac{1}{\ell_{\mathrm{P}} \bar{\square}}\left(\bar{g}_{\mu \nu}-\frac{\bar{\nabla}_{\mu} \bar{\nabla}_{\nu}}{\bar{\square}}\right) \bar{\nabla}_{\rho} h^{\rho \nu}, \\
\varphi= & \left(\bar{g}_{\mu \nu}-\frac{\bar{\nabla}_{\mu} \bar{\nabla}_{\nu}}{\bar{\square}}\right) h^{\mu \nu}, \\
\chi= & \frac{1}{3}\left(4 \frac{\bar{\nabla}_{\mu} \bar{\nabla}_{\nu}}{\square}-\bar{g}_{\mu \nu}\right) h^{\mu \nu .}
\end{aligned}
$$

If we consider the regime in which $R \ll \gamma \ell_{\mathrm{P}}^{2} R^{2}$, namely the purely de Sitter (inflationary) phase, it is easy to see that the background spacetime is well approximated by the de Sitter line element,

$$
\begin{aligned}
\mathrm{d} s^{2} & =\bar{g}_{\mu \nu} \mathrm{d} x^{\mu} \mathrm{d} x^{\nu} \\
& =-\left(1-\frac{r^{2}}{L_{\Lambda}^{2}}\right) \mathrm{d} t^{2}+\left(1-\frac{r^{2}}{L_{\Lambda}^{2}}\right)^{-1} \mathrm{~d} r^{2}+r^{2} \mathrm{~d} \Omega^{2}
\end{aligned}
$$


Besides, in this regime we have that $f(R) \simeq \gamma \ell_{\mathrm{P}}^{2} R^{2}$, thus the corresponding equations of motion reduce to

$$
\left(2 R_{\mu \nu}-\frac{1}{2} g_{\mu \nu} R\right) R+2\left(g_{\mu \nu} \square-\nabla_{\mu} \nabla_{\nu}\right) R=0 .
$$

Expanding these equations of motion over the de Sitter background, and making use of the decomposition $(5.45)$ together with the gauge fixing $\bar{\nabla}^{\mu} h_{\mu \nu}=(1 / 4) \bar{\nabla}_{\nu} h$, we find that the latter propagate only a tensor and a scalar mode according to

$$
\begin{aligned}
& \square\left(\square+\frac{4}{L_{\Lambda}^{2}}\right) \varphi=0, \\
& \left(\square-\frac{2}{L_{\Lambda}^{2}}\right) h_{\mu \nu}^{\perp}=0 .
\end{aligned}
$$

What we gain from this result is that the tensor mode propagates freely and it possesses an effective mass $m_{\mathrm{eff}}=\sqrt{2} \hbar / L_{\Lambda} \simeq \epsilon_{\Lambda}$, in accordance with the prediction of the corpuscular model (5.15), whereas the scalar degree of freedom shows a tachyonic behaviour that reflects the instability of this phase.

Now, we wish to gather more information about the behaviour of the system when we find ourselves in a quasi-de Sitter phase. In this scenario we will have to consider perturbation of the general equations (5.44) over this unknown quasi-de Sitter backgroud $\bar{g}_{\mu \nu}$. Without entering too much in the details of the computation (see [156]), from the trace of (5.44), together with the decomposition (5.45), we find the equation for the propagating scalar degree of freedom,

$$
\bar{\square}\left[\bar{\square}-\left(\frac{M^{2}}{\ell_{\mathrm{P}}^{2} m_{\mathrm{P}}^{2}}-\frac{\bar{R}}{3}\right)\right] \varphi-\frac{1}{3} \bar{\nabla}^{\mu} \bar{R} \bar{\nabla}_{\mu} \varphi=0 .
$$

Moreover, taking profit of an ad hoc gauge fixing condition introduced in 156

$$
\bar{\nabla}^{\lambda} h_{\lambda \nu}-\frac{1}{2} \bar{\nabla}_{\nu} h=\frac{1}{\bar{R}}\left[\bar{\nabla}_{\nu}\left(\bar{R}^{\lambda \rho} h_{\lambda \rho}-\frac{1}{2} \bar{R} h\right)+h_{\lambda \nu} \bar{\nabla}^{\lambda} \bar{R}\right],
$$

from the expansion of the general equations of motion we also infer that,

$$
\begin{aligned}
\square h_{\mu \nu}^{\perp} & +\frac{2 \gamma \ell_{\mathrm{P}}^{2}}{1+2 \gamma \ell_{\mathrm{P}}^{2} R} \bar{\nabla}{ }_{\lambda} h_{\mu \nu}^{\perp} \bar{\nabla}^{\lambda} \bar{R}+\bar{R}_{\mu}^{\lambda} h_{\lambda \nu}^{\perp} \\
& +\bar{R}_{\nu}^{\lambda} h_{\mu \lambda}^{\perp}-\frac{\bar{R}\left(2+5 \gamma \ell_{\mathrm{P}}^{2} \bar{R}\right)}{3\left(1+2 \gamma \ell_{\mathrm{P}}^{2} \bar{R}\right)} h_{\mu \nu}^{\perp}-\bar{C}_{\mu \lambda \nu}^{\rho} h_{\rho}^{\perp \lambda}=0 .
\end{aligned}
$$

The major result of this second analysis is that the dynamics of the scalar field shifts from a tachyonic behaviour to an oscillatory one at the threshold "background curvature" $\bar{R}_{\mathrm{c}}=3 M^{2} / \ell_{\mathrm{P}}^{2} m_{\mathrm{P}}^{2}$. This is in perfect accordance with the idea that the Einstein-Hilbert part of the action 5.21 behaves as a perturbation, whose role is to drive the model 
out of the inflationary phase. Besides, this picture further supports a more domestic interpretation of the inflaton field. In other words, there is no real need to introduce a new particle ad hoc since the inflationary scenario is taken care of by the dynamics of the cosmological condensate of gravitons 155,156. This represents a key difference from previous proposals 154,175 , where the inflaton field was included as a necessary external input.

\subsection{Cold dark matter and corpuscular dark force}

In the last few decades, an enormous amount of observational evidences, ranging from the study of the distribution of matter at large scales to galaxy rotation curves (see e.g. $16-20$ and references therein), seem to suggest the existence of an exotic form of matter. This alleged new component of our Universe is usually referred to as dark matter [20] because of its peculiar feature of not interacting with electromagnetic radiation as well as for its (almost) imperceptible pressure. Besides, the existence of this sort of dark sector was also strongly supported by the fact that the expected gravitational effect due to ordinary matter is extremely far from being suitable to account for the structure formation in the early Universe 176, 177.

In principle, black holes could explain some of the phenomenology discussed above. Indeed, they do not emit electromagnetic radiation (if not for the Hawking effect which is, in any case, sub-dominant with respect to the cosmic microwave background) and they also interact gravitationally with baryonic matter. However, unless we consider primordial black holes [178 181], the observable one do not seem to add up to properly serve as dark matter.

Another possibility consist in assuming that this dark matter might ultimately be made of new massive stable particles able to interact strongly enough with gravity and extremely weakly with the rest of the particles belonging to the Standard Model. Of course, there is a plethora of proposed new particles with these feature, such as axions [182], Kaluza-Klein excitations [183], sterile neutrinos [184] or weakly interacting massive particles (also known as WIMPs) [185], just to quote a few examples.

Because of the large variety of different approaches to the problem of dark matter, a few remarks are in order. Assuming dark matter to be non-baryonic, then we can assemble the various proposals into two (or three) classes |176|, respectively: cold and hot dark matter (some authors even include an intermediate class, called warm dark matter). With hot dark matter we refer to low-mass neutral particles that travel with ultra-relativistic velocities, whereas with cold dark matter we mean uncharged massive particles moving with non-relativistic velocities. An example of "hot" component is represented by neutrinos, whose effectiveness is still largely debated [176], whereas examples of the "cold" class are given by primordial black holes and WIMPs. At this point it is important to observe that there are several indication that hot (and warm) dark matter might ultimately be disfavoured 176, 186, thus leaving the role of key ingredient in the standard model of Big Bang cosmology ( $\Lambda \mathrm{CDM}$ model) to cold dark matter $(\mathrm{CDM})$. 
Now, the $\Lambda$ CDM model, together with the theory of cosmological inflation, represents the cornerstone of our modern description of the Universe at cosmological and galactic scales. However, it is rather well known that this picture is not completely satisfactory 187-190 since it fails to account for the observed galaxy rotation curves [191, 192 and the Tully-Fisher relation [193], which is an empirical expression that relates the mass of a spiral galaxy to the velocity of stars far away from the galactic center. Moreover, the fact that this model requires, as a fundamental assumption, the introduction of some exotic matter contribution, i.e. cold dark matter, represents a sort of philosophical issue.

Milgrom's modified Newtonian dynamics (MOND) [194,195] is a theory that challenges the $\Lambda$ CDM model at its foundations by proposing a modification of Newton's laws, at galactic scales, to properly account for experimental observations. In this framework, the Tully-Fisher relation is explained by assuming that, for regions of a galaxy far from its core, the gravitational acceleration perceived by a test particle is given by

$$
a_{\mathrm{MOND}}(r)=\sqrt{\frac{a_{\mathrm{B}}(r)}{6 L_{\Lambda}}},
$$

where

$$
a_{\mathrm{B}}(r)=\frac{G_{\mathrm{N}} m_{\mathrm{B}}(r)}{r^{2}}
$$

is the modulus of the expected (Newtonian) radial acceleration that would be caused by the baryonic mass $m_{\mathrm{B}}=m_{\mathrm{B}}(r)$ inside a sphere of radius $r$.

In recent years there have been various attempts to explain (5.55) in the framework of emerging theories of gravity. One of the most relevant proposals is Verlinde's entropic approach [196], according to which the laws of gravity emerging from quantum entanglement, at galactic scales, contain an additional dark gravitational force which may explain the phenomenology commonly attributed to dark matter, thus reproducing the MOND acceleration. An alternative picture is, instead, offered by the corpuscular model [157, 158. The key idea, as we shall discuss in details later on in this Section, is that an additional dark force, responsible for this peculiar phenomenology, can emerge as a reaction of the cosmological (dark energy) condensate of gravitons (DEC, for short) to the local presence of baryonic matter. What makes this picture particularly intriguing is that, as for cosmic inflation, it does not require the introduction of any exotic ingredients to reproduce both the effects commonly ascribed to cold dark matter and the MOND behaviours at galactic scales.

So far we have seen that the corpuscular model suggests that, at both cosmological and Newtonian scales, the relevant number of gravitons responsible for the dynamics of the system scales holographically, that is

$$
N_{\mathrm{G}}(r) \sim \frac{m^{2}(r)}{m_{\mathrm{P}}^{2}} \sim \frac{r^{2}}{\ell_{\mathrm{P}}^{2}}, \quad \text { for } r \simeq L_{\Lambda} \text { and } r \simeq R_{\mathrm{B}},
$$

where $L_{\Lambda}$ denotes the de Sitter horizon and $R_{\mathrm{B}}$, such that $R_{H} \lesssim R_{\mathrm{B}} \ll L_{\Lambda}$ with $R_{\mathrm{H}}=$ $2 G_{\mathrm{N}} m_{\mathrm{B}}$, is the typical size around which the gravitational interaction sourced by a baryonic 
matter distribution of mass $m_{\mathrm{B}}(r)$, inside a sphere of radius $r$, is well approximated by Newtonian physics. For the sake of argument, let us interpret $N_{\mathrm{G}}\left(L_{\Lambda}\right)$ as the total number of gravitons inside the visible Universe. Then, $N_{\mathrm{G}}\left(R_{\mathrm{B}}\right)$ can be understood as the number of gravitons that respond locally to the presence of the baryonic matter sources of mass $m_{\mathrm{B}}$. These $N_{\mathrm{G}}\left(R_{\mathrm{B}}\right)$ gravitons would then change their energy from $\epsilon_{\Lambda}$ to some $\epsilon_{\mathrm{G}}\left(R_{\mathrm{B}}\right)$ in order to enforce the Newtonian dynamics at the local level. This is exactly the underlying idea that will lead to the emergence of the corpuscular dark force.

Despite its success in modelling self-gravitating systems of astrophysical size as well as regions comparable with the size of the cosmological horizon, it is rather clear that the holographic regime (5.57) cannot be carried along throughout the whole range of scales. A first indication comes from the fact that the two holographic regimes at small and very large scales are indeed different, since they describe two very distinct physical scenarios (a starlike object and an exponentially expanding Universe, respectively). A second motivation comes from the fact that, locally, without the presence of baryonic matter, the DEC - responsible for the emergence of the de Sitter structure - cannot be considered as a whole, but just as a background medium with constant energy density $\rho_{\Lambda}=\Lambda / 8 \pi G_{\mathrm{N}} \sim m_{\mathrm{P}} / \ell_{\mathrm{P}} L_{\Lambda}^{2}$. Thus, the total energy inside a spherical region of radius $r$ is given by,

$$
m_{\mathrm{G}}(r) \simeq \frac{4 \pi}{3} \rho_{\Lambda} r^{3} \sim \frac{m_{\mathrm{P}} r^{3}}{L_{\Lambda}^{2} \ell_{\mathrm{P}}}=N_{\mathrm{G}}(r) \epsilon_{\Lambda},
$$

from which we infer that the number of gravitons contained in this region shows an extensive behaviour,

$$
N_{\mathrm{G}}(r) \simeq \frac{r^{3}}{L_{\Lambda} \ell_{\mathrm{P}}^{2}},
$$

i.e. it scales like the volume of the region. This suggests the existence of a new infrared scale $R_{\mathrm{B}}<r_{0}<L_{\Lambda}$, where the behaviour of gravity departs from the holographic regime. Thus, at this point, it is fairly reasonable to assume that, when dealing with scales of size $r$ with $R_{\mathrm{B}} \ll r \ll L_{\Lambda}$, in absence of baryonic matter, the graviton state features the extensive scaling $(5.59$ ).

Let us now consider a DEC of size $L_{\Lambda}$ made of $N_{\Lambda}$ constituent gravitons. If we now place $N_{\mathrm{B}}$ baryonic particles, each one of mass $\mu$, on top of the DEC in a very diluted manner, the latter will react to the presence of matter by shrinking the size of its horizon. Indeed, in geometric terms, we are effectively turning our spacetime into a Schwarzschild-de Sitter space, i.e. given the generic static spherically symmetric line element

$$
\mathrm{d} s^{2}=-f(r) \mathrm{d} t^{2}+\frac{\mathrm{d} r^{2}}{f(r)}+r^{2} \mathrm{~d} \Omega^{2}
$$

we are (statically) changing the metric function $f(r)$ according to

$$
f(r)=1-\frac{r^{2}}{L_{\Lambda}^{2}} \quad \longrightarrow \quad f(r)=1-\frac{r^{2}}{L_{\Lambda}^{2}}-\frac{2 G_{\mathrm{N}} \mu}{r},
$$


from which we can infer that the cosmological horizon now becomes $L_{\mathrm{Hor}} \sim L_{\Lambda}-G_{\mathrm{N}} \mu$. Alternatively, in a corpuscular perspective, we have that the Hamiltonian constraint (5.13) gets modified by the addition of matter $\mathcal{H}_{\mathrm{M}} \simeq N_{\mathrm{B}} \mu$, leading to the new constraint

$$
U_{\mathrm{N}}+U_{\mathrm{PN}}+N_{\mathrm{B}} \mu \simeq 0,
$$

with $U_{\mathrm{N}} \simeq N \epsilon_{\Lambda}=-N \ell_{\mathrm{P}} m_{\mathrm{P}} / L_{\Lambda}$ and $U_{\mathrm{PN}}=N^{3 / 2} \ell_{\mathrm{P}}^{2} m_{\mathrm{P}} / L_{\Lambda}^{2}$, which yields

$$
L_{\Lambda} \simeq \sqrt{N} \ell_{\mathrm{P}}+N_{\mathrm{B}} \ell_{\mathrm{P}} \mu / m_{\mathrm{P}}+\mathcal{O}\left(N^{-1}\right) .
$$

where $N$ is the number of gravitons that remain in the DEC. This tell us that the condensate must respond to the presence of this homogeneous matter by changing the graviton number $N_{\Lambda}$ into $N$ which results in a tiny shrinkage of the cosmological horizon, which now is given by $L_{\mathrm{Hor}}=\sqrt{N} \ell_{\mathrm{P}}$.

It is then interesting to see what happens if the baryonic matter starts to clump. For simplicity, let us assume that these $N_{\mathrm{B}}$ particles assemble together into a spherically symmetric distribution of matter of mass $m_{\mathrm{B}}(r)=N_{\mathrm{B}}(r) \mu$. Now the DEC will react to the presence of matter causing, as we shall show in details, the phenomenology commonly attributed to dark matter together with the correct form of the MOND acceleration. We will then interpret this reaction of the DEC as an emerging dark force. The key assumption that we make here is that only a (small) fraction of the gravitons in the DEC will be affected by the local matter, which is fairly reasonable given the negligible corrections shown in (5.63). This implies that the DEC will not react at the full cosmological scale $L_{\mathrm{Hor}}$, but only at a local scale $r$. Moreover, since we are dealing with a spherically symmetric matter distribution, the baryonic matter of mass $m_{\mathrm{B}}$ will pull some gravitons out of the DEC from inside the sphere of radius $r$ with a dark energy mass given by $M=M(r)$.

A first way to derive the MOND acceleration within the corpuscular model was proposed in [157|. The idea is based on the fact that the modulus of the Newtonian gravitational acceleration felt by a test particle is given by

$$
a(r)=\frac{G_{\mathrm{N}} m}{r^{2}} \sim \frac{\epsilon_{\mathrm{G}}(r)^{2}}{m_{\mathrm{P}}^{2} \ell_{\mathrm{P}}} \sqrt{N_{\mathrm{eff}}},
$$

where $m$ is the mass of the self-gravitating system while $N_{\text {eff }}$ is the number of gravitons with $\lambda_{\mathrm{G}} \simeq r$ involved in the interaction between the collective state and the test particle at $r$. If we now consider the reaction of the DEC, of total energy $m_{\Lambda}$, due to the presence of a clumped baryonic matter source of mass $m_{\mathrm{B}}(r)$, such that $m_{\mathrm{B}} \ll m_{\Lambda}$, then the number of gravitons that remain in the cosmological condensate can be estimated as

$$
N_{\mathrm{DE}} \simeq \frac{\left(m_{\Lambda}-m_{\mathrm{B}}\right)^{2}}{m_{\mathrm{P}}^{2}},
$$

these remaining gravitons are therefore the one that account for the expansion of the Universe, reproducing the expected effects commonly attributed to dark energy. Now, if we denote by $N_{\mathrm{N}} \simeq m_{\mathrm{B}}^{2} / m_{\mathrm{P}}^{2}$ the fraction of gravitons pulled out of the DEC, which is now 
responsible for the local Newtonian dynamics induced by the baryonic source, then it is easy to see that

$$
N_{\Lambda}-N_{\mathrm{DE}} \simeq \frac{2 m_{\mathrm{B}} m_{\Lambda}}{m_{\mathrm{P}}^{2}}-N_{\mathrm{N}} \sim \frac{L_{\Lambda} m_{\mathrm{B}}}{\ell_{\mathrm{P}} m_{\mathrm{P}}}-N_{\mathrm{N}}
$$

where we used $N_{\Lambda} \simeq m_{\Lambda}^{2} / m_{\mathrm{P}}^{2}$. The last expression then allows us to interpret,

$$
N_{\mathrm{DF}} \simeq \frac{L_{\Lambda} m_{\mathrm{B}}}{\ell_{\mathrm{P}} m_{\mathrm{P}}}
$$

as the number of gravitons responsible for the interaction between the baryonic matter and what remains of the DEC. One can therefore guess the number of non-condensed constituents $N_{\mathrm{DF}}(r)$ that provide a contribution to the gravitational acceleration of a particle at distance $r$ from the center of the galaxy. Indeed, if we choose,

$$
N_{\mathrm{DF}}(r) \sim \frac{r^{2} m_{\mathrm{B}}(r)}{\ell_{\mathrm{P}} m_{\mathrm{P}} L_{\Lambda}},
$$

then, plugging this expression into (5.64) and recalling $\epsilon_{\mathrm{G}}(r) \simeq \hbar / r$, one finds

$$
a_{\mathrm{DF}}(r) \sim \sqrt{\frac{G_{\mathrm{N}} m_{\mathrm{B}}(r)}{L_{\Lambda} r^{2}}} \sim \sqrt{\frac{a_{\mathrm{B}}(r)}{L_{\Lambda}}},
$$

that matches the MOND formula up to a factor 1/6. However, a proper justification for 5.68) can only be given by means of an argument based on energy balance.

Let us now derive the MOND acceleration from a simple energy balance and its relation with the Hamiltonian constraint of gravity [158]. Once the matter starts clumping, the corresponding energy changes to

$$
\mathcal{H}_{\mathrm{M}}=m_{\mathrm{B}}+\mathcal{E}_{\mathrm{B}}
$$

where $\mathcal{E}_{\mathrm{B}}$ keeps track of the total kinetic energy of the matter and of all energy contributions coming from non-gravitational interactions. Whereas, since some gravitons will be pulled out of the DEC in response to the local presence of matter, the gravitational Hamiltonian constraint function reads

$$
\mathcal{H}_{\mathrm{G}}=H_{\mathrm{DEC}}+H_{\mathrm{BG}}+H_{\mathrm{DF}},
$$

where $H_{\mathrm{DEC}}$ is the energy of the (new) DEC, after pulling out a small fraction of its constituents, $\mathcal{H}_{\mathrm{BG}}$ is the Newtonian gravitational interaction amongst the $N_{\mathrm{B}}$ baryons mediated by gravitons i.e.

$$
H_{\mathrm{BG}}=-\frac{G_{\mathrm{N}} m_{\mathrm{B}}^{2}}{R_{\mathrm{B}}} \simeq-N_{\mathrm{N}} \frac{\hbar}{R_{\mathrm{B}}},
$$


and $H_{\mathrm{DF}}$ denotes the energy due to the gravitational interaction between the baryonic source and the portion of the DEC contained inside the sphere of radius $r$, of total mass $M(r)$ (dark energy contained inside the sphere). Specifically, we have that

$$
H_{\mathrm{DF}}=-\frac{G_{\mathrm{N}} m_{\mathrm{B}} M(r)}{r} .
$$

It is now important to observe that we can rewrite 5.73 in terms of an effective dark force mass $m_{\mathrm{DF}}$, by assuming that

$$
\frac{G_{\mathrm{N}} m_{\mathrm{B}} M(r)}{r} \simeq \frac{G_{\mathrm{N}} m_{\mathrm{DF}}^{2}}{r},
$$

i.e. denoting $m_{\mathrm{DF}} \simeq m_{\mathrm{B}} M(r)$. Besides, it is easy to see that

$$
N_{\mathrm{DF}}(r) \simeq \frac{m_{\mathrm{DF}}^{2}}{m_{\mathrm{P}}^{2}} \simeq \frac{m_{\mathrm{B}} M(r)}{m_{\mathrm{P}}^{2}},
$$

furthermore recalling that $M(r) \simeq \bar{N}_{\mathrm{G}}(r) \epsilon_{\mathrm{G}}(r)$ with $\bar{N}_{\mathrm{G}}(r)$ is the number of gravitons in the extensive regime (5.59), it is straightforward to conclude that,

$$
M(r) \simeq \frac{r^{2}}{G_{\mathrm{N}} L_{\Lambda}},
$$

which implies,

$$
N_{\mathrm{DF}}(r)=\frac{m_{\mathrm{B}} r^{2}}{\ell_{\mathrm{P}} m_{\mathrm{P}} L_{\Lambda}},
$$

which matches exactly the guessed value (5.68). Thus, we can conclude that

$$
a_{\mathrm{DF}}(r) \sim \frac{G_{\mathrm{N}} m_{\mathrm{DF}}}{r^{2}} \sim \sqrt{\frac{a_{\mathrm{B}}(r)}{L_{\Lambda}}} \sim \frac{\epsilon_{\mathrm{G}}(r)^{2}}{m_{\mathrm{P}}^{2} \ell_{\mathrm{P}}} \sqrt{N_{\mathrm{DF}}(r)} .
$$

It is important to stress that the emergence of this dark force effect, in the corpuscular picture, is strictly related to the competition between the holographic and extensive regimes. Indeed, an heuristic derivation of the MOND acceleration can be obtained, in this framework, by focussing solely on this feature, see [158 for details. Besides, it is also possible to reconcile this corpuscular picture with an effective metric theory. Specifically, it was shown [157] that these emergent laws of gravity can be framed as an effective anisotropic fluid description of an infrared-modified theory of gravity.

To conclude, it is interesting to observe that the energy balance argument, summarized here, actually allows us to estimate the ratio between the apparent dark matter mass distribution and baryonic matter. Now, if we denote by $U_{\mathrm{DF}}$ the energy content due to the "dark gravitons", it can be expressed in terms of the graviton number $N_{\mathrm{DF}}$ according to

$$
U_{\mathrm{DF}} \simeq-N_{\mathrm{DF}} \epsilon_{\mathrm{G}}(r) \simeq-N_{\mathrm{DF}} \frac{\hbar}{r}
$$


within a sphere of radius $r$. On the other hand, the $\Lambda \mathrm{CDM}$ requires $U_{\mathrm{DF}}$ to account for the interaction of an apparent dark matter mass $m_{\mathrm{DM}}$ with the baryonic source of mass $m_{\mathrm{B}}$, as well as for its self-interaction. Mathematically this is expressed by the condition,

$$
U_{\mathrm{DF}}=-\frac{G_{\mathrm{N}} m_{\mathrm{DM}} m_{\mathrm{B}}}{r}-\frac{G_{\mathrm{N}} m_{\mathrm{DM}}^{2}}{r}
$$

If we then put together $(5.79)$ and $(5.80)$, and we recall that $N_{\mathrm{DF}} \sim N_{\Lambda}-N_{\mathrm{DE}}$, one can easily conclude (see 158 for all the details of the computation)

$$
\frac{m_{\mathrm{DM}}}{m_{\mathrm{B}}} \simeq 5.77
$$

that, despite coming from order of magnitude computations, is incredibly close to the prediction of the $\Lambda$ CDM model $[197-201]$. 


\section{Chapter 6}

\section{Conclusions and outlook}

It's not beginning the story that I

fear, it's not knowing how it will end.

Francis Underwood

The formulation of a consistent quantum theory of gravity represents one of the major open problems in theoretical physics. Indeed, despite the many experimental validations of both quantum field theory and general relativity, they appear to be mutually incompatible at a fundamental level. Nonetheless, despite the fact that the quest for quantum gravity remains one of the most compelling research lines of our time, it is natural to wonder whether the unravel of its fundamental structure is really needed in order to appreciate the observable physical phenomena that might occur in our Universe. Indeed, one could argue that if we were able to formulate an effective quantum theory of the gravitational interaction which unifies all experimental evidences into a single consistent framework, then such a theory would carry a flavour of quantum gravity.

In this Thesis we have argued that an example of such an effective theory is provided by the corpuscular theory of gravity. Thus, let us now summarise the main points addressed in this work.

First, in Chapter 2 we have reviewed the basic principles on which both the semiclassical and EFT approaches to gravity are erected. Specifically, after stressing how black hole thermodynamics naturally begs for the introduction of a certain degree of quantumness in the classical theory of gravitation, we have presented some aspects of quantum field theory on curved spacetimes, with particular regard for the derivation of the Hawking effect. Next we have shown how the Hawking process fixes the ambiguity in the definition of the Bekenstein-Hawking entropy, while implying also the emergence of the information paradox. After that, we have underlined several issues coming from the quantization of the weak field expansion of the Einstein-Hilbert action. On this matter, we have also recalled that, in this framework, the predictability of the theory is inevitably lost at the Planck scale. This is due to the fact that the effective theory at hand is non-renormalizable.

In Chapter 3, we have recalled some of the key ideas behind the EFT perspective on non-renormalizability. In particular, we have stressed that a non-renormalizable field 
theory can indeed serve as an accurate effective low-energy model. Afterwards, we have observed that the quantum field theoretic formulation of gravity (emerging form weak field expansion of the Einstein-Hilbert action) can still provide an accurate description of scattering processes involving a center of mass energy way smaller than the Planck mass. Beyond that limit, all terms in the perturbative expansion of scattering amplitudes become relevant and the system enters a strong coupling regime. After this general discussion, we have introduced the classicalization scheme as a clever way to get around this issue.

In this Chapter we have also provided a quick and dirty review of the key features of the horizon quantum mechanics formalism, with particular regard for the spherically symmetric and axisymmetric cases. In this context, we have also taken the opportunity to stress that one of the main consequences of Example 1 is that the "single constituent" description does not work that well for astrophysical black holes. Consequently, this remark hints at a more strict relation between the HQM and the corpuscular scenario.

In Chapter 4 we have laid down the groundwork for the formulation of the corpuscular model of black holes. Specifically, we have shown that a black hole can be understood as a self-sustained quantum state by means of an effective scalar theory for the toy gravitons. Then, taking advantage of the key results in Section 4.1, we have presented a corpuscular description of the gravitational collapse based on an energy balance argument related to the Hamiltonian constraint of general relativity. In Section 4.3, we have then improved on the latter analysis by establishing an effective theory for the gravitational potential of a static spherical symmetric system up to the first post-Newtonian correction. After that, we have also provided a detailed discussion of the likelihood for causal structures of the Kerr and Hayward types to be realized in corpuscular gravity. To perform this analysis we have employed the quantum harmonic potential as an effective description of the binding potential for the constituent gravitons and then we have applied the HQM formalism. Finally, we have concluded the Chapter by studying the behaviour of corpuscular black holes in $(1+1)$-dimensional spacetimes.

In Chapter 5 we have provided a generalization of the energy balance analysis, originally introduced in Chapter 4, to cosmology. Specifically, we have presented a corpuscular description of the de Sitter space, in relation with both the cosmological constant term and quadratic gravity scenario. Again, this description has been based on an energy balance emerging from the Hamiltonian constraint of cosmology, namely the one responsible for the first Friedman equation. Then, by extending this argument to the Starobinsky model, we have been able to interpret the slow-roll scenario as an effect coming from the balance between the Newtonian and post-Newtonian energy contributions to the total Hamiltonian constraint. Afterwards, we have observed that once this balance is broken the system leaves the (quasi-)de Sitter phase, i.e. the Universe stops inflating at an exponential rate.

Note that one of the main features of this model is that we are not forced to add any exotic matter, such as the inflaton, to realize the inflationary scenario. Secondly, this model has only one free parameter, namely the number of constituent gravitons in the early Universe, whose value can ultimately be inferred from the CMB data.

Continuing on with this corpuscular interpretation of the de Sitter space, we have been able to provide an alternative justification for some phenomenological evidences which 
are usually accounted for in terms of gravitational effects due to the presence of dark matter. Specifically, in Section 5.3, we have shown that if we think of the dark energy component of the Universe as the result of a cosmological condensate of gravitons, then the typical dynamics generated by the presence of cold dark matter can be re-interpreted as the response of the cosmological condensate to the localized presence of baryonic matter.

To sum up, the corpuscular theory of gravity represents an intriguing new approach capable of unifying several phenomena, from the quantum black hole regime up to galactic and cosmological scales, in a single framework. However, many questions concerning the underlying formulation of this theory remain unanswered. For example, we still lack a proper rigorous description of bound states in quantum field theory, without which one cannot really chart the deep implications of the classicalization scheme in the dynamical process of black hole formation. Then, our poor grasp of this point reflects in a lack of a precise description of the emergence of the classical metric structure of the spacetime as the result of the superposition of many soft quanta. 


\section{Appendix A}

\section{A note on the Fierz-Pauli kinetic term}

The Fierz-Pauli kinetic term 202, 203], often referred to as the massless Fierz-Pauli lagrangian in this work, can be constructed as the most general Lorentz-invariant action for a massless Spin-2 field, i.e.

$$
\mathcal{L}_{\mathrm{FP}, \mathrm{kin}}=a \partial_{\mu} h_{\nu \rho} \partial^{\mu} h^{\nu \rho}+b \partial_{\mu} h_{\nu \rho} \partial^{\nu} h^{\mu \rho}+c \partial_{\mu} h \partial_{\rho} h^{\mu \rho}+d \partial_{\mu} h \partial^{\mu} h,
$$

with $a, b, c, d \in \mathbb{R} \backslash\{0\}$. The corresponding equations of motion are given by

$$
\mathcal{O}_{\alpha \beta \mu \nu} h^{\mu \nu}=a \square h_{\alpha \beta}+2 b \partial^{\mu} \partial_{(\alpha} h_{\beta) \mu}+c \partial_{(\alpha} \partial_{\beta)} h+c \eta_{\alpha \beta} \partial^{\mu} \partial^{\lambda} h_{\mu \lambda}+2 d \eta_{\alpha \beta} \square h=0 .
$$

Now, since we want to reproduce Einstein's gravity, we wish to recover $\mathcal{O}_{\alpha \beta \mu \nu} h^{\mu \nu} \propto T_{\alpha \beta}$. Thus, if we recall that the conservation of energy implies $\partial^{\alpha} T_{\alpha \beta}=0$, we immediately get the gauge condition,

$$
\partial^{\alpha}\left(\mathcal{O}_{\alpha \beta \mu \nu} h^{\mu \nu}\right)=0
$$

which entails

$$
2 a=-b=c=-2 d,
$$

hence the coefficients of the Fierz-Pauli kinetic term are not uniquely defined. Moreover, one can rewrite A.1 as

$$
\mathcal{L}_{\mathrm{FP}, \text { kin }}^{(a)}=2 a\left(\frac{1}{2} \partial_{\mu} h_{\nu \rho} \partial^{\mu} h^{\nu \rho}-\partial_{\mu} h_{\nu \rho} \partial^{\nu} h^{\mu \rho}+\partial_{\mu} h \partial_{\rho} h^{\mu \rho}-\frac{1}{2} \partial_{\mu} h \partial^{\mu} h\right) .
$$

Note that the definition that we have employed in 2.29 corresponds to the case with $a=-1 / 2$. It is also worth remarking that another representation of the Fierz-Pauli kinetic term, which is often found in the literature, is given by $a=-1 / 4$. Specifically, let us consider the Einstein-Hilbert action (2.28), together with its weak field expansion. From A.5 it is straightforward to see that

$$
S_{\mathrm{EH}} \simeq \frac{1}{4 \chi} \int \mathrm{d}^{4} x \mathcal{L}_{\mathrm{FP}, \mathrm{kin}}^{(-1 / 2)}=\frac{1}{2 \chi} \int \mathrm{d}^{4} x \mathcal{L}_{\mathrm{FP}, \mathrm{kin}}^{(-1 / 4)}
$$




\section{Acknowledgments}

I would like to take this opportunity to thank the various individuals to whom I am indebted, not only for their help in preparing this thesis, but also for their support and guidance throughout the last three years.

First and foremost, I wish to express my deepest gratitude to my supervisors, Roberto Casadio and Gia Dvali, for encouraging me to pursue this research line and for providing me with a friendly and insightful guidance throughout my graduate studies.

I wish to thank Stefan Hofmann and Mariafelicia De Laurentis for agreeing to be part of my PhD committee, as well as Ivo Sachs and Alexandr Yu. Kamenchtchik for accepting to serve as substitute examiners.

I would also like to show my gratitude to my collaborators: Andrea Giugno, Ivano Colombaro, Francesco Mainardi, Luigi Frunzo, Octavian Micu, Michele Lenzi, Mariano Cadoni, Matteo Tuveri, Wolfgang Mück, Jonas Mureika, Alexis Helou, Rehana Rahim and Victor Alfonzo Diaz. None of this would have been possible without them.

Finally, I would like extend my gratitude to those who have not directly been part of my academic life, but that have been, nonetheless, of central importance in my life. First, and foremost, I wish to thank my parents, Antonella and Maurizio, for their unrelenting support and for teaching me the value of things. Besides, a special word of gratitude goes to my sisters, Giulia and Giorgia, who have had to deal with my unending rants and other oddities. I would also like to thank some of my dearest friends, in particular Simone, Margherita, and Martina, for their constant encouragement and unlimited support. Last but not least, I wish to dedicated the last few words of this thesis to my girlfriend Claudia. I owe her my deepest gratitude for her perpetual inspiration and support, and for putting up with both the long distance and my endless uninteresting updates on the latest traumatic turn of events. 


\section{Bibliography}

[1] A. Einstein, "The Foundation of the General Theory of Relativity," Annalen Phys. 49 no. 7, (1916) 769-822.

[2] A. Einstein, "On the electrodynamics of moving bodies," Annalen Phys. 17 (1905) 891-921. [Annalen Phys.14,194(2005)].

[3] H. Minkowski, "Die Grundgleichungen für die electromagnetischen Vorgänge in bewegten Körpern," Gott. Nach. (1908) 53-111. [Mathematische Annalen 68,472(1910)].

[4] I. Newton, Philosophice Naturalis Principia Mathematica. England, 1687.

[5] Virgo, LIGO Scientific Collaboration, B. P. Abbott et al., "Observation of Gravitational Waves from a Binary Black Hole Merger," Phys. Rev. Lett. 116 no. 6, (2016) 061102, arXiv:1602.03837 [gr-qc].

[6] Virgo, LIGO Scientific Collaboration, B. P. Abbott et al., "GW151226: Observation of Gravitational Waves from a 22-Solar-Mass Binary Black Hole Coalescence," Phys. Rev. Lett. 116 no. 24, (2016) 241103, arXiv: 1606.04855 [gr-qc].

[7] K. Schwarzschild, "On the gravitational field of a mass point according to Einstein's theory," Sitzungsber. Preuss. Akad. Wiss. Berlin (Math. Phys.) 1916 (1916) 189-196, arXiv:physics/9905030 [physics].

[8] K. Schwarzschild, "On the gravitational field of a sphere of incompressible fluid according to Einstein's theory," Sitzungsber. Preuss. Akad. Wiss. Berlin (Math. Phys.) 1916 (1916) 424-434, arXiv:physics/9912033 [physics.hist-ph].

[9] D. Finkelstein, "Past-Future Asymmetry of the Gravitational Field of a Point Particle," Phys. Rev. 110 (1958) 965-967.

[10] M. D. Kruskal, "Maximal extension of Schwarzschild metric," Phys. Rev. 119 (1960) 1743-1745.

[11] S. W. Hawking, "Particle Creation by Black Holes," Commun. Math. Phys. 43 (1975) 199-220. 
[12] A. Einstein, "Cosmological Considerations in the General Theory of Relativity," Sitzungsber. Preuss. Akad. Wiss. Berlin (Math. Phys.) (1917) 142-152.

[13] A. Einstein and W. de Sitter, "On the Relation between the Expansion and the Mean Density of the Universe," Proc. Nat. Acad. Sci. 18 (1932) 213-214.

[14] E. Hubble, "A relation between distance and radial velocity among extra-galactic nebulae," Proc. Nat. Acad. Sci. 15 (1929) 168-173.

[15] M. S. Turner, "Dark matter and dark energy in the universe," ASP Conf. Ser. 165 (1999) 431, arXiv:astro-ph/9811454 [astro-ph].

[16] K. G. Begeman, "H I rotation curves of spiral galaxies. I - NGC 3198," Astron. Astrophys. 223 (1989) 47-60.

[17] F. Zwicky, "On the Masses of Nebulae and of Clusters of Nebulae," Astrophys. J. 86 (1937) 217-246.

[18] VIRGO Consortium Collaboration, A. Jenkins, C. S. Frenk, F. R. Pearce, P. A. Thomas, J. M. Colberg, S. D. M. White, H. M. P. Couchman, J. A. Peacock, G. Efstathiou, and A. H. Nelson, "Evolution of structure in cold dark matter universes," Astrophys. J. 499 (1998) 20, arXiv:astro-ph/9709010 [astro-ph].

[19] D. Clowe, M. Bradac, A. H. Gonzalez, M. Markevitch, S. W. Randall, C. Jones, and D. Zaritsky, "A direct empirical proof of the existence of dark matter," Astrophys. J. 648 (2006) L109-L113, arXiv:astro-ph/0608407 [astro-ph].

[20] K. Garrett and G. Duda, "Dark Matter: A Primer," Adv. Astron. 2011 (2011) 968283, arXiv:1006.2483 [hep-ph].

[21] A. H. Guth, "The Inflationary Universe: A Possible Solution to the Horizon and Flatness Problems," Phys. Rev. D23 (1981) 347-356.

[22] A. D. Linde, "A New Inflationary Universe Scenario: A Possible Solution of the Horizon, Flatness, Homogeneity, Isotropy and Primordial Monopole Problems," Phys. Lett. 108B (1982) 389-393.

[23] A. A. Starobinsky, "A New Type of Isotropic Cosmological Models Without Singularity," Phys. Lett. B91 (1980) 99-102.

[24] A. A. Starobinsky, "Spectrum of relict gravitational radiation and the early state of the universe," JETP Lett. 30 (1979) 682-685.

[25] V. F. Mukhanov, H. A. Feldman, and R. H. Brandenberger, "Theory of cosmological perturbations. Part 1. Classical perturbations. Part 2. Quantum theory of perturbations. Part 3. Extensions," Phys. Rept. 215 (1992) 203-333. 
[26] A. D. Linde and V. F. Mukhanov, "Nongaussian isocurvature perturbations from inflation," Phys. Rev. D56 (1997) R535-R539, arXiv:astro-ph/9610219 [astro-ph].

[27] A. H. Guth and S. Y. Pi, "Fluctuations in the New Inflationary Universe," Phys. Rev. Lett. 49 (1982) 1110-1113.

[28] L. Kofman, A. D. Linde, and A. A. Starobinsky, "Towards the theory of reheating after inflation," Phys. Rev. D56 (1997) 3258-3295, arXiv:hep-ph/9704452 [hep-ph].

[29] J. M. Bardeen, B. Carter, and S. W. Hawking, "The Four laws of black hole mechanics," Commun. Math. Phys. 31 (1973) 161-170.

[30] S. W. Hawking, "Black holes in general relativity," Commun. Math. Phys. 25 (1972) 152-166.

[31] D. Sudarsky and R. M. Wald, "Mass formulas for stationary Einstein Yang-Mills black holes and a simple proof of two staticity theorems," Phys. Rev. D47 (1993) R5209-R5213, arXiv:gr-qc/9305023 [gr-qc].

[32] R. M. Wald, "The First law of black hole mechanics," in Directions in General Relativity: An International Symposium in Honor of the 60th Birthdays of Dieter Brill and Charles Misner College Park, Maryland, May 27-29, 1993, pp. 358-366. 1993. arXiv:gr-qc/9305022 [gr-qc].

[33] H. S. Reall, "Black holes,". Lecture notes for the course on Black Holes in Part III of the Cambridge Mathematical Tripos.

[34] D. Christodoulou, "Reversible and irreversible transforations in black hole physics," Phys. Rev. Lett. 25 (1970) 1596-1597.

[35] W. Israel, "Third Law of Black-Hole Dynamics: A Formulation and Proof," Phys. Rev. Lett. 57 no. 4, (1986) 397.

[36] R. M. Wald, "The thermodynamics of black holes," Living Rev. Rel. 4 (2001) 6, arXiv:gr-qc/9912119 [gr-qc].

[37] J. D. Bekenstein, "Black holes and the second law," Lett. Nuovo Cim. 4 (1972) 737-740.

[38] W. Israel, "Event horizons in static vacuum space-times," Phys. Rev. 164 (1967) 1776-1779.

[39] W. Israel, "Event horizons in static electrovac space-times," Commun. Math. Phys. 8 (1968) 245-260. 
[40] J. D. Bekenstein, "Black holes and entropy," Phys. Rev. D7 (1973) 2333-2346.

[41] S. Hollands and R. M. Wald, "Quantum fields in curved spacetime," Phys. Rept. 574 (2015) 1-35, arXiv:1401.2026 [gr-qc].

[42] T. Jacobson, "Introduction to quantum fields in curved space-time and the Hawking effect," in Lectures on quantum gravity. Proceedings, School of Quantum Gravity, Valdivia, Chile, January 4-14, 2002, pp. 39-89. 2003. arXiv:gr-qc/0308048 [gr-qc].

[43] R. L. Arnowitt, S. Deser, and C. W. Misner, "The Dynamics of general relativity," Gen. Rel. Grav. 40 (2008) 1997-2027, arXiv:gr-qc/0405109 [gr-qc].

[44] R. M. Wald, "On Particle Creation by Black Holes," Commun. Math. Phys. 45 (1975) 9-34.

[45] S. W. Hawking, "Breakdown of Predictability in Gravitational Collapse," Phys. Rev. D14 (1976) 2460-2473.

[46] S. W. Hawking, "Information loss in black holes," Phys. Rev. D72 (2005) 084013, arXiv:hep-th/0507171 [hep-th].

[47] D. N. Page, "Particle Emission Rates from a Black Hole: Massless Particles from an Uncharged, Nonrotating Hole," Phys. Rev. D13 (1976) 198-206.

[48] D. N. Page, "Information in black hole radiation," Phys. Rev. Lett. 71 (1993) 3743-3746, arXiv:hep-th/9306083 [hep-th].

[49] J. W. York, Jr., "Dynamical Origin of Black Hole Radiance," Phys. Rev. D28 (1983) 2929.

[50] A. Strominger, "Black hole entropy from near horizon microstates," JHEP 02 (1998) 009, arXiv:hep-th/9712251 [hep-th].

[51] D. N. Page, "Average entropy of a subsystem," Phys. Rev. Lett. 71 (1993) 1291-1294, arXiv:gr-qc/9305007 [gr-qc].

[52] J. D. Bekenstein, "A Universal Upper Bound on the Entropy to Energy Ratio for Bounded Systems," Phys. Rev. D23 (1981) 287.

[53] J. D. Bekenstein, "Entropy bounds and black hole remnants," Phys. Rev. D49 (1994) 1912-1921, arXiv:gr-qc/9307035 [gr-qc].

[54] G. 't Hooft and M. J. G. Veltman, "One loop divergencies in the theory of gravitation," Ann. Inst. H. Poincare Phys. Theor. A20 (1974) 69-94.

[55] M. H. Goroff and A. Sagnotti, "Quantum gravity at two loops," Phys. Lett. 160B (1985) 81-86. 
[56] M. H. Goroff and A. Sagnotti, "The Ultraviolet Behavior of Einstein Gravity," Nucl. Phys. B266 (1986) 709-736.

[57] J. F. Donoghue, "General relativity as an effective field theory: The leading quantum corrections," Phys. Rev. D50 (1994) 3874-3888, arXiv: gr-qc/9405057 $[\mathrm{gr}-\mathrm{qc}]$.

[58] J. F. Donoghue, "Leading quantum correction to the Newtonian potential," Phys. Rev. Lett. 72 (1994) 2996-2999, arXiv:gr-qc/9310024 [gr-qc].

[59] J. F. Donoghue, "Introduction to the effective field theory description of gravity," in Advanced School on Effective Theories Almunecar, Spain, June 25-July 1, 1995. 1995. arXiv:gr-qc/9512024 [gr-qc].

[60] S. Deser, "Selfinteraction and gauge invariance," Gen. Rel. Grav. 1 (1970) 9-18, arXiv:gr-qc/0411023 [gr-qc].

[61] S. Deser, "Gravity from self-interaction redux," Gen. Rel. Grav. 42 (2010) 641-646, arXiv:0910.2975 [gr-qc].

[62] M. J. G. Veltman, "Quantum Theory of Gravitation," Conf. Proc. C7507281 (1975) 265-327.

[63] B. S. DeWitt, "Quantum Theory of Gravity. 1. The Canonical Theory," Phys. Rev. 160 (1967) 1113-1148.

[64] B. S. DeWitt, "Quantum Theory of Gravity. 2. The Manifestly Covariant Theory," Phys. Rev. 162 (1967) 1195-1239.

[65] B. S. DeWitt, "Quantum Theory of Gravity. 3. Applications of the Covariant Theory," Phys. Rev. 162 (1967) 1239-1256.

[66] J. F. Donoghue, M. M. Ivanov, and A. Shkerin, "EPFL Lectures on General Relativity as a Quantum Field Theory," arXiv:1702.00319 [hep-th]. EPFL Lectures on General Relativity as a Quantum Field Theory.

[67] D. Lovelock, "The Einstein tensor and its generalizations," J. Math. Phys. 12 (1971) 498-501.

[68] N. E. J. Bjerrum-Bohr, J. F. Donoghue, and B. R. Holstein, "Quantum corrections to the Schwarzschild and Kerr metrics," Phys. Rev. D68 (2003) 084005 , arXiv:hep-th/0211071 [hep-th]. [Erratum: Phys. Rev.D71,069904(2005)].

[69] J. F. Donoghue, "When Effective Field Theories Fail," PoS EFT09 (2009) 001, arXiv:0909.0021 [hep-ph].

[70] R. Casadio, "Localised particles and fuzzy horizons: A tool for probing Quantum Black Holes," arXiv:1305.3195 [gr-qc]. 
[71] R. Casadio and F. Scardigli, "Horizon wave-function for single localized particles: GUP and quantum black hole decay," Eur. Phys. J. C74 no. 1, (2014) 2685. arXiv:1306.5298 [gr-qc].

[72] R. Casadio, O. Micu, and F. Scardigli, "Quantum hoop conjecture: Black hole formation by particle collisions," Phys. Lett. B732 (2014) 105-109, arXiv:1311.5698 [hep-th].

[73] R. Casadio, "Horizons and non-local time evolution of quantum mechanical systems," Eur. Phys. J. C75 no. 4, (2015) 160, arXiv:1411.5848 [gr-qc].

[74] R. Casadio, O. Micu, and D. Stojkovic, "Inner horizon of the quantum Reissner-Nordström black holes," JHEP 05 (2015) 096, arXiv: 1503.01888 $[\mathrm{gr}-\mathrm{qc}]$.

[75] R. Casadio, O. Micu, and D. Stojkovic, "Horizon Wave-Function and the Quantum Cosmic Censorship," Phys. Lett. B747 (2015) 68-72, arXiv:1503.02858 [gr-qc].

[76] R. Casadio, R. T. Cavalcanti, A. Giugno, and J. Mureika, "Horizon of quantum black holes in various dimensions," Phys. Lett. B760 (2016) 36-44, arXiv:1509.09317 [gr-qc].

[77] R. Casadio, A. Giugno, and O. Micu, "Horizon quantum mechanics: A hitchhiker's guide to quantum black holes," Int. J. Mod. Phys. D25 no. 02, (2016) 1630006, arXiv:1512.04071 [hep-th]

[78] R. Casadio, A. Giugno, and A. Giusti, "Global and Local Horizon Quantum Mechanics," Gen. Rel. Grav. 49 no. 2, (2017) 32, arXiv:1605.06617 [gr-qc].

[79] R. Casadio, A. Giugno, A. Giusti, and O. Micu, "Horizon Quantum Mechanics of Rotating Black Holes," Eur. Phys. J. C77 no. 5, (2017) 322, arXiv:1701.05778 $[\mathrm{gr}-\mathrm{qc}]$.

[80] A. Giusti, "Horizon Quantum Mechanics: spherically symmetric and rotating sources," J. Phys. Conf. Ser. 942 no. 1, (2017) 012013, arXiv:1709.10348 $[\mathrm{gr}-\mathrm{qc}]$.

[81] A. Giugno, A. Giusti, and A. Helou, "Horizon quantum fuzziness for non-singular black holes," Eur. Phys. J. C78 no. 3, (2018) 208, arXiv:1711.06209 [gr-qc].

[82] R. Casadio, A. Giusti, and R. Rahim, "Horizon Quantum Mechanics for spheroidal sources," EPL 121 no. 6, (2018) 60004, arXiv:1801.05621 [gr-qc].

[83] R. Rahim, A. Giusti, and R. Casadio, "The marginally trapped surfaces in spheroidal spacetimes," Int. J. Mod. Phys. D28 (2019) 1950021, arXiv:1804.11218 [gr-qc]. 
[84] R. Casadio, A. Giugno, A. Giusti, and M. Lenzi, "Quantum Formation of Primordial Black holes," arXiv:1810.05185 [gr-qc].

[85] D. Lerner and J. R. Porter, "Asymptotically simple space-time manifolds," J. Math. Phys. 15 (1974) 1416-1419.

[86] R. Penrose, "Gravitational collapse and space-time singularities," Phys. Rev. Lett. $14(1965) 57-59$.

[87] S. W. Hawking and R. Penrose, "The Singularities of gravitational collapse and cosmology," Proc. Roy. Soc. Lond. A314 (1970) 529-548.

[88] C. W. Misner and D. H. Sharp, "Relativistic equations for adiabatic, spherically symmetric gravitational collapse," Phys. Rev. 136 (1964) B571-B576.

[89] S. Hawking, "Gravitational radiation in an expanding universe," J. Math. Phys. 9 (1968) 598-604.

[90] S. A. Hayward, "Quasilocal gravitational energy," Phys. Rev. D49 (1994) 831-839, arXiv:gr-qc/9303030 [gr-qc].

[91] M. Dafermos, "Spherically symmetric space-times with a trapped surface," Class. Quant. Grav. 22 (2005) 2221-2232, arXiv:gr-qc/0403032 [gr-qc].

[92] L. B. Szabados, "Quasi-Local Energy-Momentum and Angular Momentum in General Relativity," Living Rev. Rel. 12 (2009) 4.

[93] R. P. Kerr, "Gravitational field of a spinning mass as an example of algebraically special metrics," Phys. Rev. Lett. 11 (1963) 237-238.

[94] G. C. Debney, R. P. Kerr, and A. Schild, "Solutions of the Einstein and Einstein-Maxwell Equations," J. Math. Phys. 10 (1969) 1842.

[95] B. Carter, "Global structure of the Kerr family of gravitational fields," Phys. Rev. 174 (1968) 1559-1571.

[96] B. Carter, "Axisymmetric Black Hole Has Only Two Degrees of Freedom," Phys. Rev. Lett. 26 (1971) 331-333.

[97] D. C. Robinson, "Uniqueness of the Kerr black hole," Phys. Rev. Lett. 34 (1975) 905-906.

[98] E. Fermi, "An attempt of a theory of beta radiation. 1.," Z. Phys. 88 (1934) 161-177.

[99] M. Maggiore, A Modern introduction to quantum field theory, vol. 12 of Oxford Master Series in Physics. Oxford University Press, 1 ed., 2005. 
[100] K. G. Wilson, "The Renormalization Group and Strong Interactions," Phys. Rev. D3 (1971) 1818.

[101] K. G. Wilson and J. B. Kogut, "The Renormalization group and the epsilon expansion," Phys. Rept. 12 (1974) 75-200.

[102] G. Dvali and C. Gomez, "Self-Completeness of Einstein Gravity," arXiv:1005.3497 [hep-th].

[103] G. Dvali, G. F. Giudice, C. Gomez, and A. Kehagias, "UV-Completion by Classicalization," JHEP 08 (2011) 108, arXiv:1010.1415 [hep-ph].

[104] G. Dvali, "Strong Coupling and Classicalization," Subnucl. Ser. 53 (2017) 189-200, arXiv:1607.07422 [hep-th].

[105] G. Dvali, C. Gomez, and A. Kehagias, "Classicalization of Gravitons and Goldstones," JHEP 11 (2011) 070, arXiv:1103.5963 [hep-th].

[106] G. Dvali, C. Gomez, R. S. Isermann, D. Lüst, and S. Stieberger, "Black hole formation and classicalization in ultra-Planckian $2 \rightarrow \mathrm{N}$ scattering," Nucl. Phys. B893 (2015) 187-235, arXiv: 1409.7405 [hep-th].

[107] G. Dvali and C. Gomez, "Black Hole's Quantum N-Portrait," Fortsch. Phys. 61 (2013) 742-767, arXiv:1112.3359 [hep-th].

[108] G. Dvali and C. Gomez, "Black Hole's 1/N Hair," Phys. Lett. B719 (2013) 419-423, arXiv:1203.6575 [hep-th].

[109] K. S. Thorne, "Nonspherical Gravitational Collapse: A Short Review,". In: Magic Without Magic: John Archibald Wheeler, A Collection of Essays in Honor of his Sixtieth Birthday. Edited by John R. Klauder. San Francisco: W.H. Freeman.

[110] E. Flanagan, "Hoop conjecture for black-hole horizon formation," Phys. Rev. D44 (1991) 2409 .

[111] D. Dou and R. Percacci, "The running gravitational couplings," Class. Quant. Grav. 15 (1998) 3449-3468, arXiv:hep-th/9707239 [hep-th].

[112] M. Reuter and F. Saueressig, "Renormalization group flow of quantum gravity in the Einstein-Hilbert truncation," Phys. Rev. D65 (2002) 065016, arXiv:hep-th/0110054 [hep-th].

[113] A. Bonanno and M. Reuter, "Cosmology of the Planck era from a renormalization group for quantum gravity," Phys. Rev. D65 (2002) 043508, arXiv:hep-th/0106133 [hep-th].

[114] G. Dvali and C. Gomez, "Black Holes as Critical Point of Quantum Phase Transition," Eur. Phys. J. C74 (2014) 2752, arXiv:1207.4059 [hep-th]. 
[115] G. Dvali, D. Flassig, C. Gomez, A. Pritzel, and N. Wintergerst, "Scrambling in the Black Hole Portrait," Phys. Rev. D88 no. 12, (2013) 124041, arXiv:1307.3458 [hep-th].

[116] G. Dvali, A. Franca, C. Gomez, and N. Wintergerst, "Nambu-Goldstone Effective Theory of Information at Quantum Criticality," Phys. Rev. D92 no. 12, (2015) 125002, arXiv:1507.02948 [hep-th].

[117] N. N. Bogolyubov, "On the theory of superfluidity," J. Phys.(USSR) 11 (1947) 23-32. [Izv. Akad. Nauk Ser. Fiz.11,77(1947)].

[118] W. Mück, "Hawking radiation is corpuscular," Eur. Phys. J. C76 no. 7, (2016) 374 arXiv:1606.01790 [hep-th].

[119] R. Casadio, A. Giugno, O. Micu, and A. Orlandi, "Black holes as self-sustained quantum states, and Hawking radiation," Phys. Rev. D90 no. 8, (2014) 084040, arXiv:1405.4192 [hep-th]

[120] R. Casadio, A. Giugno, and A. Orlandi, "Thermal corpuscular black holes," Phys. Rev. D91 no. 12, (2015) 124069, arXiv:1504.05356 [gr-qc].

[121] R. Casadio, A. Giugno, O. Micu, and A. Orlandi, "Thermal BEC black holes," Entropy 17 (2015) 6893-6924, arXiv:1511.01279 [gr-qc].

[122] M. Abramowitz and I. A. Stegun, Handbook of mathematical functions: with formulas, graphs, and mathematical tables. Dover publications, New York, 1 ed., 1964.

[123] R. Casadio, A. Giugno, and A. Giusti, "Matter and gravitons in the gravitational collapse," Phys. Lett. B763 (2016) 337-340, arXiv:1606.04744 [hep-th].

[124] R. L. Arnowitt, S. Deser, and C. W. Misner, "Dynamical Structure and Definition of Energy in General Relativity," Phys. Rev. 116 (1959) 1322-1330.

[125] R. L. Arnowitt, S. Deser, and C. W. Misner, "Canonical variables for general relativity," Phys. Rev. 117 (1960) 1595-1602.

[126] R. C. Tolman, "Static solutions of Einstein's field equations for spheres of fluid," Phys. Rev. 55 (1939) 364-373.

[127] J. R. Oppenheimer and G. M. Volkoff, "On Massive neutron cores," Phys. Rev. 55 (1939) 374-381.

[128] R. Casadio and A. Orlandi, "Quantum Harmonic Black Holes," JHEP 08 (2013) 025, arXiv:1302.7138 [hep-th].

[129] W. Mück and G. Pozzo, "Quantum portrait of a black hole with Pöschl-Teller potential," JHEP 05 (2014) 128, arXiv: 1403.1422 [hep-th]. 
[130] R. Casadio, A. Giugno, A. Giusti, and M. Lenzi, "Quantum corpuscular corrections to the Newtonian potential," Phys. Rev. D96 no. 4, (2017) 044010, arXiv:1702.05918 [gr-qc].

[131] A. Giugno, "Quantum post-Newtonian theory for corpuscular Black Holes," J. Phys. Conf. Ser. 942 no. 1, (2017) 012012, arXiv:1710.04487 [gr-qc].

[132] R. Casadio, M. Lenzi, and O. Micu, "Bootstrapping Newton Gravity," arXiv:1806.07639 [gr-qc]

[133] M. S. Madsen, "Scalar Fields in Curved Space-times," Class. Quant. Grav. 5 (1988) 627-639.

[134] J. D. Brown, "Action functionals for relativistic perfect fluids," Class. Quant. Grav. 10 (1993) 1579-1606, arXiv:gr-qc/9304026 [gr-qc].

[135] J. M. Bardeen, "Non-singular General-Relativistic Gravitational Collapse," in Proceedings of International Conference GR5, Tbilisi University Press, 1968, p. 174. 1968.

[136] R. Casadio, "Quantum gravitational fluctuations and the semiclassical limit," Int. J. Mod. Phys. D9 (2000) 511-529, arXiv:gr-qc/9810073 [gr-qc].

[137] L. Modesto and P. Nicolini, "Charged rotating noncommutative black holes," Phys. Rev. D82 (2010) 104035, arXiv: 1005.5605 [gr-qc].

[138] C. Bambi and L. Modesto, "Rotating regular black holes," Phys. Lett. B721 (2013) 329-334, arXiv: 1302.6075 [gr-qc].

[139] C. Bambi, D. Malafarina, and L. Modesto, "Non-singular quantum-inspired gravitational collapse," Phys. Rev. D88 (2013) 044009, arXiv:1305.4790 [gr-qc].

[140] V. P. Frolov, "Notes on nonsingular models of black holes," Phys. Rev. D94 no. 10, (2016) 104056, arXiv: 1609.01758 [gr-qc].

[141] D. Malafarina, "Classical collapse to black holes and quantum bounces: A review," Universe 3 no. 2, (2017) 48, arXiv:1703.04138 [gr-qc].

[142] T. De Lorenzo, A. Giusti, and S. Speziale, "Non-singular rotating black hole with a time delay in the center," Gen. Rel. Grav. 48 no. 3, (2016) 31, arXiv:1510.08828 [gr-qc]. [Erratum: Gen. Rel. Grav.48,no.8,111(2016)].

[143] S. A. Hayward, "Formation and evaporation of regular black holes," Phys. Rev. Lett. 96 (2006) 031103, arXiv:gr-qc/0506126 [gr-qc].

[144] R. B. Mann, A. Shiekh, and L. Tarasov, "Classical and Quantum Properties of Two-dimensional Black Holes," Nucl. Phys. B341 (1990) 134-154. 
[145] R. B. Mann, "Lower dimensional black holes," Gen. Rel. Grav. 24 (1992) 433-449.

[146] S. Carlip, "Quantum gravity in $2+1$ dimensions: The Case of a closed universe," Living Rev. Rel. 8 (2005) 1, arXiv:gr-qc/0409039 [gr-qc].

[147] R. B. Mann and J. R. Mureika, "(1+1)-Dimensional Entropic Gravity," Phys. Lett. B703 (2011) 167-171, arXiv:1105.5925 [hep-th]

[148] J. R. Mureika, "Primordial Black Hole Evaporation and Spontaneous Dimensional Reduction," Phys. Lett. B716 (2012) 171-175, arXiv:1204.3619 [gr-qc].

[149] S. Carlip, "Dimension and Dimensional Reduction in Quantum Gravity," Class. Quant. Grav. 34 no. 19, (2017) 193001, arXiv:1705.05417 [gr-qc].

[150] V. A. Diaz and A. Giusti, "Fractional Bosonic Strings," J. Math. Phys. 59 no. 3, (2018) 033509, arXiv:1710.09370 [hep-th].

[151] F. Scardigli, "Generalized uncertainty principle in quantum gravity from micro black hole Gedanken experiment," Phys. Lett. B452 (1999) 39-44, arXiv:hep-th/9904025 [hep-th].

[152] F. Scardigli and R. Casadio, "Generalized uncertainty principle, extra dimensions and holography," Class. Quant. Grav. 20 (2003) 3915-3926, arXiv:hep-th/0307174 [hep-th].

[153] R. Casadio, A. Giusti, and J. Mureika, "Lower dimensional corpuscular gravity and the end of black hole evaporation," arXiv:1805.10444 [gr-qc].

[154] G. Dvali and C. Gomez, "Quantum Compositeness of Gravity: Black Holes, AdS and Inflation," JCAP 1401 (2014) 023, arXiv:1312.4795 [hep-th].

[155] R. Casadio, A. Giugno, and A. Giusti, "Corpuscular slow-roll inflation," Phys. Rev. D97 no. 2, (2018) 024041, arXiv:1708.09736 [gr-qc].

[156] A. Giugno and A. Giusti, "Domestic Corpuscular Inflaton," arXiv:1806.11168 [gr-qc].

[157] M. Cadoni, R. Casadio, A. Giusti, W. Mück, and M. Tuveri, "Effective Fluid Description of the Dark Universe," Phys. Lett. B776 (2018) 242-248, arXiv:1707.09945 [gr-qc].

[158] M. Cadoni, R. Casadio, A. Giusti, and M. Tuveri, "Emergence of a Dark Force in Corpuscular Gravity," Phys. Rev. D97 no. 4, (2018) 044047, arXiv:1801.10374 [gr-qc].

[159] S. M. Carroll, Spacetime and geometry: An introduction to general relativity. San Francisco, USA: Addison-Wesley, 1 ed., 2004. 
[160] M. Blau, "General Relativity,". Lecture Notes on General Relativity, University of Bern.

[161] V. Faraoni and E. Gunzig, "Einstein frame or Jordan frame?," Int. J. Theor. Phys. 38 (1999) 217-225, arXiv : astro-ph/9910176 [astro-ph].

[162] V. Faraoni, "de Sitter space and the equivalence between $\mathrm{f}(\mathrm{R})$ and scalar-tensor gravity," Phys. Rev. D75 (2007) 067302, arXiv:gr-qc/0703044 [GR-QC].

[163] T. P. Sotiriou and V. Faraoni, "f(R) Theories Of Gravity," Rev. Mod. Phys. 82 (2010) 451-497, arXiv:0805.1726 [gr-qc].

[164] S. Capozziello, M. De Laurentis, and V. Faraoni, "A Bird's eye view of f(R)-gravity," Open Astron. J. 3 (2010) 49, arXiv:0909.4672 [gr-qc].

[165] S. Capozziello, M. De Laurentis, S. Nojiri, and S. D. Odintsov, "Classifying and avoiding singularities in the alternative gravity dark energy models," Phys. Rev. D79 (2009) 124007, arXiv:0903.2753 [hep-th]

[166] A. De Felice and S. Tsujikawa, "f(R) theories," Living Rev. Rel. 13 (2010) 3, arXiv:1002.4928 [gr-qc].

[167] S. Capozziello and M. De Laurentis, "Extended Theories of Gravity," Phys. Rept. 509 (2011) 167-321, arXiv:1108.6266 [gr-qc].

[168] M. De Laurentis, M. Paolella, and S. Capozziello, "Cosmological inflation in $F(R, \mathcal{G})$ gravity," Phys. Rev. D91 no. 8, (2015) 083531, arXiv:1503.04659 [gr-qc].

[169] Supernova Cosmology Project Collaboration, S. Perlmutter et al., "Measurements of Omega and Lambda from 42 high redshift supernovae," Astrophys. J. 517 (1999) 565-586, arXiv:astro-ph/9812133 [astro-ph].

[170] Supernova Search Team Collaboration, A. G. Riess et al., "Observational evidence from supernovae for an accelerating universe and a cosmological constant," Astron. J. 116 (1998) 1009-1038, arXiv:astro-ph/9805201 [astro-ph].

[171] Planck Collaboration, P. A. R. Ade et al., "Planck 2015 results. XX. Constraints on inflation," Astron. Astrophys. 594 (2016) A20, arXiv:1502.02114 [astro-ph.CO].

[172] R. Utiyama and B. S. DeWitt, "Renormalization of a classical gravitational field interacting with quantized matter fields," J. Math. Phys. 3 (1962) 608-618.

[173] S. D. Odintsov and I. L. Shapiro, "General relativity as the low-energy limit in higher derivative quantum gravity," Class. Quant. Grav. 9 (1992) 873-882. [Teor. Mat. Fiz.90,469(1992)]. 
[174] J. F. Donoghue and G. Menezes, "Gauge Assisted Quadratic Gravity: A Framework for UV Complete Quantum Gravity," Phys. Rev. D97 no. 12, (2018) 126005, arXiv:1804.04980 [hep-th].

[175] R. Casadio, F. Kuhnel, and A. Orlandi, "Consistent Cosmic Microwave Background Spectra from Quantum Depletion," JCAP 1509 (2015) 002, arXiv: 1502.04703 $[\mathrm{gr}-\mathrm{qc}]$.

[176] J. R. Primack, "Dark matter and structure formation," in Midrasha Mathematicae in Jerusalem: Winter School in Dynamical Systems Jerusalem, Israel, January 12-17, 1997. 1997. arXiv:astro-ph/9707285 [astro-ph].

[177] J. L. Feng, "Dark Matter Candidates from Particle Physics and Methods of Detection," Ann. Rev. Astron. Astrophys. 48 (2010) 495-545, arXiv:1003.0904 [astro-ph.CO].

[178] S. Hawking, "Gravitationally collapsed objects of very low mass," Mon. Not. Roy. Astron. Soc. 152 (1971) 75.

[179] B. J. Carr and S. W. Hawking, "Black holes in the early Universe," Mon. Not. Roy. Astron. Soc. 168 (1974) 399-415.

[180] B. J. Carr, "The Primordial black hole mass spectrum," Astrophys. J. 201 (1975) 1-19.

[181] B. J. Carr, K. Kohri, Y. Sendouda, and J. Yokoyama, "New cosmological constraints on primordial black holes," Phys. Rev. D81 (2010) 104019 arXiv:0912.5297 [astro-ph.CO].

[182] L. D. Duffy and K. van Bibber, "Axions as Dark Matter Particles," New J. Phys. 11 (2009) 105008, arXiv:0904.3346 [hep-ph].

[183] N. Arkani-Hamed, S. Dimopoulos, and G. R. Dvali, "Phenomenology, astrophysics and cosmology of theories with submillimeter dimensions and $\mathrm{TeV}$ scale quantum gravity," Phys. Rev. D59 (1999) 086004, arXiv:hep-ph/9807344 [hep-ph].

[184] A. Boyarsky, O. Ruchayskiy, and M. Shaposhnikov, "The Role of sterile neutrinos in cosmology and astrophysics," Ann. Rev. Nucl. Part. Sci. 59 (2009) 191-214, arXiv:0901.0011 [hep-ph]

[185] G. Bertone, "The moment of truth for WIMP Dark Matter," Nature 468 (2010) 389-393, arXiv:1011.3532 [astro-ph.CO].

[186] M. Viel, G. D. Becker, J. S. Bolton, and M. G. Haehnelt, "Warm dark matter as a solution to the small scale crisis: New constraints from high redshift Lyman- $\alpha$ forest data," Phys. Rev. D88 (2013) 043502, arXiv:1306.2314 [astro-ph.C0]. 
[187] A. A. Klypin, A. V. Kravtsov, O. Valenzuela, and F. Prada, "Where are the missing Galactic satellites?," Astrophys. J. 522 (1999) 82-92, arXiv:astro-ph/9901240 [astro-ph]

[188] B. Moore, S. Ghigna, F. Governato, G. Lake, T. R. Quinn, J. Stadel, and P. Tozzi, "Dark matter substructure within galactic halos," Astrophys. J. 524 (1999) L19-L22, arXiv:astro-ph/9907411 [astro-ph]

[189] M. Boylan-Kolchin, J. S. Bullock, and M. Kaplinghat, "Too big to fail? The puzzling darkness of massive Milky Way subhaloes," Mon. Not. Roy. Astron. Soc. 415 (2011) L40, arXiv:1103.0007 [astro-ph.C0].

[190] M. Boylan-Kolchin, J. S. Bullock, and M. Kaplinghat, "The Milky Way's bright satellites as an apparent failure of LCDM," Mon. Not. Roy. Astron. Soc. 422 (2012) 1203-1218, arXiv:1111.2048 [astro-ph.CO].

[191] V. C. Rubin, N. Thonnard, and W. K. Ford, Jr., "Rotational properties of 21 SC galaxies with a large range of luminosities and radii, from NGC $4605 / \mathrm{R}=4 \mathrm{kpc} /$ to UGC $2885 / \mathrm{R}=122 \mathrm{kpc} /$, , Astrophys. J. 238 (1980) 471.

[192] M. Persic, P. Salucci, and F. Stel, "The Universal rotation curve of spiral galaxies: 1. The Dark matter connection," Mon. Not. Roy. Astron. Soc. 281 (1996) 27, arXiv:astro-ph/9506004 [astro-ph].

[193] R. B. Tully and J. R. Fisher, "A New method of determining distances to galaxies," Astron. Astrophys. 54 (1977) 661-673.

[194] M. Milgrom, "A Modification of the Newtonian dynamics as a possible alternative to the hidden mass hypothesis," Astrophys. J. 270 (1983) 365-370.

[195] M. Milgrom, "MOND theory," Can. J. Phys. 93 no. 2, (2015) 107-118, arXiv:1404.7661 [astro-ph.CO].

[196] E. P. Verlinde, "Emergent Gravity and the Dark Universe," SciPost Phys. 2 no. 3, (2017) 016, arXiv: 1611.02269 [hep-th].

[197] WMAP Collaboration, D. N. Spergel et al., "First year Wilkinson Microwave Anisotropy Probe (WMAP) observations: Determination of cosmological parameters," Astrophys. J. Suppl. 148 (2003) 175-194, arXiv:astro-ph/0302209 [astro-ph].

[198] WMAP Collaboration, E. Komatsu et al., "Seven-Year Wilkinson Microwave Anisotropy Probe (WMAP) Observations: Cosmological Interpretation," Astrophys. J. Suppl. 192 (2011) 18, arXiv:1001.4538 [astro-ph.CO].

[199] A. Del Popolo, "Dark matter and structure formation a review," Astron. Rep. 51 (2007) 169-196, arXiv:0801.1091 [astro-ph]. 
[200] Planck Collaboration, P. A. R. Ade et al., "Planck 2013 results. XVI. Cosmological parameters," Astron. Astrophys. 571 (2014) A16, arXiv:1303.5076 [astro-ph.CO].

[201] Planck Collaboration, P. A. R. Ade et al., "Planck 2015 results. XIII. Cosmological parameters," Astron. Astrophys. 594 (2016) A13, arXiv:1502.01589 [astro-ph.CO].

[202] K. Hinterbichler, "Theoretical Aspects of Massive Gravity," Rev. Mod. Phys. 84 (2012) 671-710, arXiv:1105.3735 [hep-th].

[203] C. de Rham, "Massive Gravity," Living Rev. Rel. 17 (2014) 7, arXiv:1401.4173 [hep-th]. 